Preprint typeset in JHEP style - HYPER VERSION

WITS-CTP-038, UUITP-25/08

\title{
MHV, CSW and BCFW: field theory structures in string theory amplitudes
}

\author{
Rutger Boels \\ Niels Bohr International Academy, Niels Bohr Institute \\ Blegdamsvej 17, DK-2100 Copenhagen, Denmark \\ Kasper Jens Larsen \\ Niels Bohr Institute, Blegdamsvej 17, DK-2100 Copenhagen, Denmark \\ Department of Physics and Astronomy, Uppsala University, SE-75108 Uppsala, Sweden
}

\author{
Niels A. Obers \\ Niels Bohr Institute, Blegdamsvej 17, DK-2100 Copenhagen, Denmark

\begin{abstract}
Marcel Vonk
Center for Theoretical Physics, University of the Witwatersrand

1, Jan Smuts Ave, Johannesburg, South Africa
\end{abstract}

\begin{abstract}
Motivated by recent progress in calculating field theory amplitudes, we study applications of the basic ideas in these developments to the calculation of amplitudes in string theory. We consider in particular both non-Abelian and Abelian open superstring disk amplitudes in a flat space background, focusing mainly on the four-dimensional case. The basic field theory ideas under consideration split into three separate categories. In the first, we argue that the calculation of $\alpha^{\prime}$-corrections to MHV open string disk amplitudes reduces to the determination of certain classes of polynomials. This line of reasoning is then used to determine the $\alpha^{\prime 3}$-correction to the MHV amplitude for all multiplicities. A second line of attack concerns the existence of an analog of CSW rules derived from the Abelian Dirac-Born-Infeld action in four dimensions. We show explicitly that the CSW-like perturbation series of this action is surprisingly trivial: only helicity conserving amplitudes are non-zero. Last but not least, we initiate the study of BCFW on-shell recursion relations in string theory. These should appear very naturally as the UV properties of the string theory are excellent. We show that all open four-point string amplitudes in a flat background at the disk level obey BCFW recursion relations. Based on the naturalness of the proof and some explicit results for the five-point gluon amplitude, it is expected that this pattern persists for all higher point amplitudes and for the closed string.
\end{abstract}

KEywords: D-branes, Supersymmetric gauge theory, Superstrings and Heterotic Strings. 


\section{Contents}

1. Introduction 2

2. General considerations for string theory amplitudes 3

2.1 Direct calculation 3

2.2 Effective action arguments

2.3 Analytic constraints

2.4 Effective supersymmetry 6

3. Determining $\alpha^{\prime}$ corrections to the MHV amplitude 9

3.1 Symmetry constraints on the amplitude 9

3.2 A toy model: scalar variables 10

3.3 Vector variables 14

3.4 Methodology 16

3.5 Results 18

4. CSW rules for the Abelian DBI action 22

4.1 Dirac-Born-Infeld in four dimensions 22

4.2 Deriving scattering amplitudes from the DBI action 24

4.3 Helicity conserving amplitudes from DBI 27

4.4 Interpretation as (partial) CSW rules 30

5. BCFW recursion relations in string theory 31

5.1 The main BCFW idea 32

5.2 Veneziano amplitude 34

5.3 Absence of residues at infinity for four-point functions 36

5.4 The four- and five-point gluon amplitudes 38

5.5 An attempt at truncating the BCFW recursion relations 43

6. Conclusions and outlook 44

6.1 MHV, CSW and BCFW: 45

6.2 field theory structures in string theory amplitudes 47

A. Technical details relevant for section 3

B. On-shell recursion relations for the Dirac-Born-Infeld action 53

G. Poles and residues for the five-point gluon amplitude 55 


\section{Introduction}

Recently much technology has become available to calculate scattering amplitudes in fourdimensional Yang-Mills and gravitational theories. This was mainly inspired by Witten's twistor string proposal [1] and includes at tree level the formulation of new Feynman-like rules [2] as well as new recursive relations [3, 仴. Historically, the development of new technology in four-dimensional Yang-Mills theory was often motivated from string theory. However, amplitude technology has come a long way, and perhaps now is the time to reverse the reasoning. Specifically, to what extent can we find structures present in gauge theory such as MHV amplitudes, CSW rules and BCFW on-shell recursion relations directly for the string? Interestingly, string theory grew out of 'analytic S-matrix' approaches to field theory, and this is exactly the type of approach which is dominating the cutting edge of (analytic) amplitude calculations in the last few years. In this article we take a first step in applying these new field theory techniques and results to string theory amplitudes in a three-pronged attack: by studying analogs of the MHV amplitude, by manipulating the effective space-time action for Abelian fields and by investigating on-shell recursion relations.

The MHV or Parke-Taylor [5] amplitude is the scattering amplitude in four-dimensional Yang-Mills theory for a process which has 2 massless particles with a certain helicity, say -, and an arbitrary number of massless particles with the opposite helicity. It displays a remarkable simplicity. The question of $\alpha^{\prime}$ corrections to this result was first raised in a series of papers by Stieberger and Taylor [6, 0, 8]. At the disk level the leading term in the $\alpha^{\prime}$ expansion is reproduced simply by Yang-Mills theory. Even more, at that level the conformal anomalies can safely be ignored so that one can perform calculations with just a four-dimensional target space. Here the 5,6 and 7-gluon amplitudes were calculated directly from the string theory. Then, based on soft limits, Stieberger and Taylor conjectured an all-multiplicity expression for the first correction of the MHV amplitudes at order $\alpha^{\prime 2}$. The result displays a similar simplicity as the Yang-Mills MHV amplitude. However, it is clear that the worldsheet-dominated methods employed in these papers in their current form do not really 'scale' well with particle multiplicity, so new input is needed. We propose in this paper that this input can be motivated from the four-dimensional target space point of view, with some very basic physical considerations and string theory computations. In the course of the investigations, several interesting structures will be uncovered.

Apart from the desire to learn more about string theory, one should keep in mind that there are several technical interconnections known between field theory and string theory which also motivate the investigation reported here. For example, the integration over Feynman parameters in a generic loop integral in field theory resembles the integration over vertex operator insertions in string theory. As another example, the $\alpha^{\prime 2}$ correction to the MHV amplitude resembles up to an almost trivial factor the 1-loop all plus amplitude [6]. To connect to more recent work, we show in this article the (not-so-surprising) result that the string theory MHV amplitude must be proportional to the field theory MHV amplitude $^{1}$. However, this is the same reasoning as used to argue that the $\mathcal{N}=4 \mathrm{MHV}$

\footnotetext{
${ }^{1}$ This was also noticed recently in [9].
} 
amplitude is proportional to the tree level MHV amplitude to all loop orders. The proportionality function there is the subject of much debate as it can be calculated both at weak (e.g. [10]) and strong (e.g [11]) coupling and has a surprising connection to Wilson loops, integrability and other interesting structures in field and string theory. In this context it is intriguing that the (four-point) proportionality function in the flat background case considered in this paper obeys for instance a maximal transcendentality principle.

This article is structured as follows: in section 2 some general considerations are discussed and some techniques common in field theory will be shown to apply directly to string theory. These observations will be put to work in section 3, where we obtain the all-multiplicity MHV amplitude up to order $\alpha^{\prime 3}$. In the next section, we switch tracks and study what can be learned about Abelian amplitudes from studying the Dirac-Born-Infeld action in four dimensions. Surprisingly, we are able to show diagrammatically that the DBI action only generates helicity conserving amplitudes by obtaining very simple CSWlike Feynman rules. In particular, the only MHV amplitude which does not vanish is the one for 4 photons. A final section 5 studies the subject of $\mathrm{BCFW}$ recursion relations in string theory. We show that these seem to arise naturally and involve stringy concepts as 'duality', 'Regge-behavior' and 'resonances' which go back all the way to the birth of string theory, which ties neatly into the observation in the opening paragraph of this section. The practical use of these relations is still quite limited at present, but must be seen as an invitation to join the fun. Conclusions round off the main presentation, and some technicalities are dealt with in several appendices.

\section{General considerations for string theory amplitudes}

In this section we review various known string and field theory facts about amplitude calculations and show that some arguments known in field theory carry over directly to the open superstring theory setting. The actual amplitudes can be obtained in basically two different ways: by direct calculation, or by constructing the effective action through other methods and calculating the Feynman diagrams. Aside from these calculations there are constraints on the amplitudes from soft and collinear limits, and from supersymmetry. As a matter of notation, we will write gluon amplitudes in 'color-ordered' form,

$$
A_{\text {full }}\left(p_{1}, p_{2}, \ldots p_{n}\right)=g^{n-2} \sum_{\sigma \in S_{n} / \mathbb{Z}_{n}} A_{\text {sub }}(\sigma(1), \ldots, \sigma(n)) \operatorname{Tr}\left(T^{a_{\sigma(1)}} \ldots T^{a_{\sigma(n)}}\right),
$$

where $T^{a_{i}}$ are the generators of the gauge group (see e.g. [12]). This is of course very natural from the point of view of the underlying disk diagram. The quantity $A_{\text {sub }}$ is referred to as a subamplitude. It is easy to see that this amplitude must be cyclic.

\subsection{Direct calculation}

Gluon (or photon) amplitudes in open string theory can of course be calculated through the usual operator representation, see e.g. [13]. Vertex operators are inserted along the boundary of the disk and the position of three of them can be fixed by global conformal invariance, whereas the rest are integrated over. Actually, it is these integrations which 
form in general the obstacle to calculating the scattering amplitudes in full generality, and simple concrete results through this direct avenue of attack are limited to the three and for gluon scattering amplitudes, which read

$$
A_{3}\left(1^{-}, 2^{-}, 3^{+}\right)=\frac{\langle 12\rangle^{4}}{\langle 12\rangle\langle 23\rangle\langle 31\rangle},
$$

and

$$
A_{4}\left(1^{-}, 2^{-}, 3^{+}, 4^{+}\right)=\frac{\langle 12\rangle^{4}}{\langle 12\rangle\langle 23\rangle\langle 34\rangle\langle 41\rangle} \frac{\Gamma\left(1-\alpha^{\prime} s\right) \Gamma\left(1-\alpha^{\prime} t\right)}{\Gamma\left(1-\alpha^{\prime}(s+t)\right)},
$$

in color ordered form. Note that these amplitudes are calculated in superstring theory; in the bosonic string there will for instance be an extra contribution $\left(\mathcal{O}\left(\alpha^{\prime}\right)\right)$ to the three particle amplitude. The four-point amplitude is of course closely related to the classical Veneziano amplitude [14]. As an aside: it is remarkable that the $\alpha^{\prime}$ expansion of this fourpoint gluon superstring amplitude seems to satisfy a 'maximal transcendentality' principle: the dimensionless constant multiplying the $\alpha^{\prime}$ contribution appears to be a product of $\zeta$ functions whose arguments add to $i$.

Progress for explicit expressions for the higher point amplitudes has been very limited, although recent work has led to forms for up to the seven-point amplitude 15, 16, 6, 7, 8] and those which are related to these by supersymmetry, i.e. with up to four gluinos and/or adjoint scalars as external states. However, further progress along this line seems hard. The problem seems to be that a high point amplitude will involve a sum over various integrals over positions of vertex operators. Both the sum over the integrals as well as the integrals themselves seem very complicated. However, see appendix A of [9] for some recent promising progress with regard to the sum at least.

\subsection{Effective action arguments}

One reason one would like to calculate gluon amplitudes is to reconstruct the string theory effective action which can then be taken off-shell to study all kinds of physical effects. However, the effective action can also be obtained by various other methods. For Abelian fields, there is for instance the classic result (see [17] and references therein) that the effective action takes the form

$$
S[A]=S_{\mathrm{DBI}}[A]+S_{\text {derivatives }}[A],
$$

where the leading-in-derivatives piece is the famous Dirac-Born-Infeld (DBI) action,

$$
S_{\mathrm{DBI}}=-1+\frac{1}{\pi^{2} g_{s} \alpha^{4}} \int d^{10} x \sqrt{-\operatorname{det}\left(\eta_{\mu \nu}+\alpha^{\prime} \pi F_{\mu \nu}\right)} .
$$

Note that from a perturbative point of view, this action contains an infinite series of vertices. The sub-leading pieces in (2.4) contain derivatives acting on the field strength tensor. In the Abelian case, there is a clean gauge-independent derivative expansion. This expansion however breaks down in the non-Abelian case since two derivatives can always be traded for a field strength tensor, e.g. for an adjoint field $H$,

$$
\left[D_{\mu}, D_{\nu}\right] H=\left[F_{\mu \nu}, H\right] .
$$



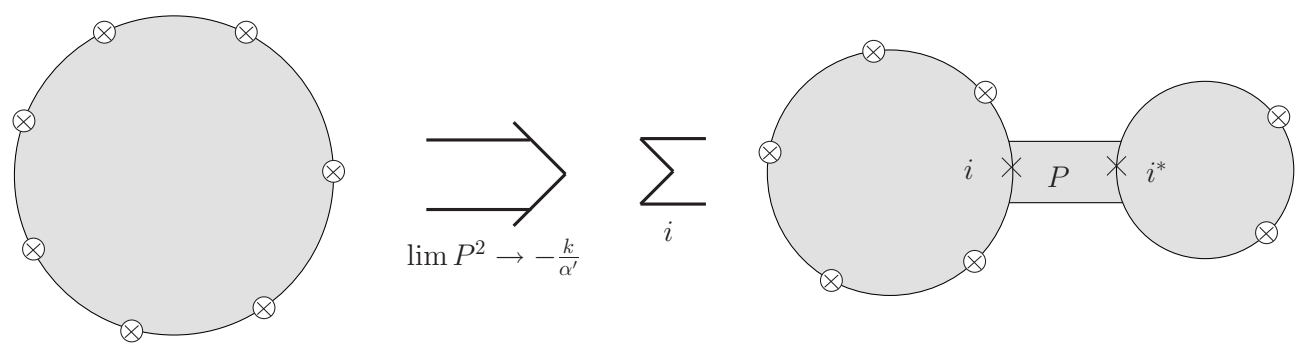

Figure 1: Conformal symmetry elucidates a certain kinematical limit with $k$ a non-negative integer. The sum runs over all string states at this particular mass level.

Therefore in the non-Abelian case one needs to find the complete effective action in one go, which makes the problem of determining it much harder. The effective action is, however, known up to order $\alpha^{\prime 3}[18]$.

To our best knowledge, deriving scattering amplitudes from string theory effective actions usually proceeds by laborious Feynman diagram calculations. We will see in section 4. however, that already in the Abelian case these calculations can be streamlined quite considerably in four dimensions.

\subsection{Analytic constraints}

Since explicit computation of string theory gluon scattering amplitudes seems to be hard by either method mentioned above, let us focus on the analytic behavior of the amplitudes as momentum invariants vanish. For color-ordered amplitudes as written in (2.1), the quantity $A_{\text {sub }}$ has a certain specific ordering of the gluons. As a consequence, the sub-amplitude can only have poles if a momentum invariant constructed out of consecutive gluon momenta vanishes. That is, the amplitudes can only have poles of the form

$$
\text { poles } \sim \frac{1}{\left(p_{i}+p_{i+1}+\ldots+p_{j}\right)^{2}+\frac{k}{\alpha^{\prime}}},
$$

for some mass $m_{l}^{2}=\frac{k}{\alpha^{\prime}}$ with $k$ a non-negative integer. In particular, it can be zero.

Actually, the form of the poles is easy to see directly from the disk diagram. As indicated in figure 1, we can always, by conformal transformations, map the disk into a structure which has a very long strip stretching between two disks. As the strip between the disks becomes very long, the states which can travel between the disks start to become closer and closer to the mass-shell. This will lead through the string theory propagator to poles of the form indicated above in (2.7). In fact, for a general amplitude there can be infinitely many different massive states which can travel through the strip corresponding to the full tower of states of the string. It is easy to see that the residue at a certain pole where $\left(p_{1}+p_{2}+\ldots+p_{j}\right)^{2} \rightarrow-\frac{k}{\alpha^{\prime}}$ will be exactly two disk amplitudes,

$$
A_{n}(1,2,3 \ldots, n) \rightarrow \sum_{i} \frac{A_{j+1}(1,2, \ldots, j, i) A_{n-j+1}(i, j+1, \ldots, n)}{\left(p_{1}+p_{2}+\ldots+p_{j}\right)^{2}+\frac{k}{\alpha^{\prime}}}
$$


where one has to sum over all the different intermediate states of the string at a certain mass level. Note that this sum splits into a sum over different particles and their quantum numbers (such as angular momentum). A discussion of this can be found in e.g. 13].

As a special case, one can consider the massless poles of a gluon amplitude. These are interesting since the residues at these poles are again amplitudes which only contain gluons. When a three-gluon disk factors out, so in the kinematic limit

$$
\left(p_{j}+p_{j+1}\right)^{2}=2 p_{j} \cdot p_{j+1} \rightarrow 0
$$

for some index $j$ equation (2.8) simplifies considerably. In this particular case, the residue of this kinematic pole consists of a three gluon superstring disk diagram (2.2) times an $n-1$ gluon disk diagram. Since the three gluon diagram is actually independent of $\alpha^{\prime}$, it is seen that the general structure of the residue is precisely the same as in tree level Yang-Mills theory. In particular, this implies that the superstring theory has the exact same collinear singularities as the field theory ${ }^{2}$. Note that the technical difficulty that the three-point Yang-Mills amplitudes vanish in Minkowski signature can easily be avoided by generalizing to complex momenta. The singularities can conveniently by described by the so-called splitting functions (see e.g. 12]). Let $S\left(i^{ \pm}, j^{ \pm},(j+1)^{ \pm}\right)$denote the splitting function with intermediate state $i$, and external states $j$ and $j+1$ and the indicated helicity. In the collinear limit (2.9),

$$
p_{j}^{\mu}=z Q^{\mu} \quad, \quad p_{j+1}^{\mu}=(1-z) Q^{\mu} \quad, \quad Q^{2}=0,
$$

the splitting functions are easily derived from, for instance, the MHV amplitude and read

$$
\begin{array}{ccc}
S\left(i^{+} j^{+}(j+1)^{+}\right)=0 & , & S\left(i^{-} j^{+}(j+1)^{+}\right)=\frac{1}{\sqrt{(1-z) z}} \frac{1}{\langle j, j+1\rangle} \\
S\left(i^{+} j^{+}(j+1)^{-}\right)=\frac{(1-z)^{2}}{\sqrt{(1-z) z}} \frac{1}{\langle j, j+1\rangle} & , & S\left(i^{-} j^{+}(j+1)^{-}\right)=\frac{(1-z)^{2}}{\sqrt{(1-z) z}} \frac{1}{[j, j+1]} \\
S\left(i^{+} j^{-}(j+1)^{-}\right)=\frac{1}{\sqrt{(1-z) z}} \frac{1}{[j, j+1]} & , & S\left(i^{-} j^{-}(j+1)^{-}\right)=0 .
\end{array}
$$

An even simpler singularity arises in the case where one of the momenta vanishes, say $p_{j} \rightarrow 0$. This so-called 'soft' singularity gives rise to a pole which is closely related to the above one, since a soft singularity is of course a special case of a collinear singularity. This is discussed in e.g chapter 6 of 19 .

\subsection{Effective supersymmetry}

As noted in [12], the form of the MHV amplitude in four-dimensional field theory can be understood at tree level through an effective supersymmetry. In particular, the supersymmetric Ward identities determine in these four dimensions [20]

$$
A(++\ldots+)=0, \quad A(++\ldots+-)=0,
$$

with an exception only for the three particle $A(++-)$ and $A(--+)$ amplitude which vanish generically only for all momenta in $\mathbb{R}^{(1,3)}$. The input in this argument is nothing

\footnotetext{
${ }^{2}$ Note that in the case of the bosonic string, there is a $\mathcal{O}\left(\alpha^{\prime}\right)$ correction to equation 2.2 which spoils this argument.
} 
more than simple representation theory of on-shell $\mathcal{N}=1$ space-time supersymmetry and the absence of helicity-violating fermion amplitudes ${ }^{3}$. Just as the derivation of these Ward identities is independent of the loop counting parameter $\hbar$ in loop computations in ordinary supersymmetric Yang-Mills theory, it is actually independent of $\alpha^{\prime}$ (and $g_{s}$ for that matter). Hence (2.12) hold to arbitrary order in $\alpha^{\prime}$, again in four dimensions. Note that these are the only amplitudes in open superstring theory which are known to all orders. This is the target space counterpart of the worldsheet based argument in [8].

Equation (2.12) implies that the next helicity amplitude in line, the MHV amplitude, cannot have multi-particle massless kinematic poles: on such poles the amplitude would factorize into two pieces, one of which is bound to vanish. This is because the propagator always respects helicity and with our 'ingoing' helicity convention, it is of the form ' +- '. It is therefore clear that it has only two-particle collinear poles. These have a universal form,

$$
A\left(+\ldots-{ }_{i} \ldots-{ }_{j} \ldots+\right) \sim \frac{D_{i j}^{(N)}}{\langle 12\rangle\langle 23\rangle \cdots\langle N 1\rangle},
$$

which can easily be checked to encode all the collinear poles in the splitting functions in equation (2.11). Effective $\mathcal{N}=4$ supersymmetry also requires that the numerator of this amplitude reads

$$
D_{i j}^{(N)}=\langle i j\rangle^{4} Q^{(N)},
$$

where $Q^{(N)}$ is a function of the momenta. More precisely, supersymmetry requires that

$$
\frac{A\left(+\ldots-{ }_{i} \ldots-{ }_{j} \ldots+\right)}{\langle i j\rangle^{4}}=\frac{A\left(+\ldots-{ }_{i} \ldots-{ }_{j+1} \ldots+\right)}{\langle i(j+1)\rangle^{4}}
$$

so that this particular combination does not depend on $i$ or $j$. Dimensional analysis now fixes $Q^{(N)}$ to be (mass-)dimensionless and have spinor weight zero. Note incidentally that this is the same argument as is used in loop calculations in $\mathcal{N}=4$ Yang-Mills theory to argue that the MHV amplitude is proportional to the tree amplitude to all loop orders. The analog of the proportionality function $Q^{(N)}$ in that case is the subject of much ongoing debate because it can be calculated at both strong and weak coupling.

In the case under study here, since all the massless kinematic poles of the amplitude have been expressed in (2.13) and we are considering the effective theory of massless gluons only, $Q^{(N)}$ has to be a polynomial in the momenta. There can be no further kinematic poles as there are no more propagating particles. At the classical level in pure Yang-Mills theory there is no natural parameter with the dimension of mass. This fixes $Q^{(N)}$ to be a particle number independent constant in this case, which can then be determined by considering the 3 -point amplitudes. On the other hand, in string theory there is a dimensionful constant $\alpha^{\prime}$ of dimension -2 . In general the function $Q^{(N)}$ will have numerous poles for the exchange of massive particles. However, if we consider an $\alpha^{\prime}$ expansion, these will have been integrated

\footnotetext{
${ }^{3}$ The absence of helicity-violating fermion amplitudes in open superstring theory follows from charge conjugation invariance. To see this note that the vertex operators of space-time fermions involve spin fields $\Theta_{\alpha}$ satisfying the $\mathrm{OPE} \Theta_{\alpha}(z) \Theta_{\beta}(w) \propto C_{\alpha \beta}$ where $C$ is the charge conjugation matrix, which is non-zero only for spinors of opposite chirality.
} 
out, so in the effective theory there are only massless propagating particles. Therefore when considering such an expansion, $Q^{(N)}$ is again a polynomial in the external momenta. Explicitly, $Q^{(N)}$ may be expanded in $\alpha^{\prime}$ as

$$
Q^{(N)}=\sum_{n \geq 0}\left(\alpha^{\prime}\right)^{n} Q_{n}^{(N)}
$$

where $Q_{n}^{(N)}$ are homogeneous polynomials in the momenta of the external particles of mass dimension $2 n$. The coefficients $Q_{n}^{(N)}$ in the color ordered amplitude now inherit certain properties from the parent amplitude. We will see in section 3 how these properties and some known results for low external particle numbers fix the form of the $\alpha^{\prime}$ corrections to the MHV amplitude for any number of external particles up to order $\alpha^{3}$.

\section{Twistor space interpretation}

The above form of the string theory MHV amplitude can be summarized by the following statement: in the low energy limit the ordinary superstring theory MHV amplitudes at the disk level localize on a holomorphic line with derivative of delta function support. This follows easily from Witten's original insight [1], see also [21]. In split $(2,2)$ signature this can be seen by considering the amplitude to be a functional of all the spinor momenta,

$$
A\left(p_{\dot{\alpha}}^{i}, p_{\alpha}^{i}, \ldots\right) \sim \delta\left(\sum_{i} p^{i}\right) \frac{\langle r s\rangle^{4}\left(Q\left(p_{\dot{\alpha}}^{i}, p_{\alpha}^{i}\right)\right)}{\langle 12\rangle\langle 23\rangle \ldots\langle n 1\rangle}
$$

temporarily resurrecting an otherwise suppressed momentum conserving delta function. In split signature a lift to twistor space boils down to performing a Fourier transform with respect to all the undotted spinors,

$$
\tilde{A}\left(p_{\dot{\alpha}}^{i}, \tilde{p}_{\alpha}^{i}, \ldots\right)=\int\left(\prod_{j} d p_{\alpha}^{j} \exp ^{i\left(\left[\tilde{p}^{i} p^{j}\right]\right)}\right) A\left(p_{\dot{\alpha}}^{i}, p_{\alpha}^{j}, \ldots\right) .
$$

The momentum conserving delta function can be represented as an integral through

$$
\delta\left(\sum_{i} p_{i}\right)=\int d^{4} x \exp ^{i\left(x^{\alpha \dot{\alpha}} \sum_{i} p_{\alpha}^{i} p_{\dot{\alpha}}^{i}\right)}
$$

The only dependence on the anti-holomorphic spinors $p_{\alpha}^{i}$ in our general form of the amplitude are contained within the polynomial in the numerator. By the Fourier transform in (2.18), these occurrences can always be replaced by derivatives by the usual rule,

$$
p_{\alpha}^{i} \rightarrow-i \frac{\partial}{\partial \tilde{p}_{\alpha}^{i}} .
$$

The remaining integral over $p_{\alpha}^{i}$ can then easily be recognized as (a series of) delta function(s). The twistor space function $\tilde{A}$ will therefore have the form

$$
\tilde{A}=Q\left(p_{\dot{\alpha}}^{i},-i \frac{\partial}{\partial \tilde{p}_{\alpha}^{i}}\right) \int d^{4} x \delta\left(\tilde{p}_{\alpha}^{i}-x_{\alpha \dot{\alpha}} p^{\dot{\alpha}}\right) A_{\mathrm{MHV}}
$$


The argument of the delta functions is of course the famous incidence relation in twistor theory. This relation describes the holomorphic embedding of a $\mathbb{C P}^{1}$ (line) inside $\mathbb{C P}^{3}$, parametrized by the space-time coordinate $x$. Note that the $\alpha^{\prime}$ corrections imply that the amplitude has derivative of delta-function support on this line, with twice the number of derivatives as powers of $\alpha^{\prime}$.

Localization on a line in twistor space implies that in some sense the string theory MHV amplitudes are local in space-time. In field theory this observation was originally made by Nair [22]. Since these amplitudes are local one may wonder whether there is an action which has these amplitudes directly as vertices. In the field theory this can be derived from the usual Yang-Mills theory [23] by lifting to twistor space (see [24] for a review), and in the Abelian case this will be discussed briefly in section 4 .

\section{Determining $\alpha^{\prime}$ corrections to the MHV amplitude}

In the previous section it was argued that the form of the MHV superstring amplitude is determined up to a polynomial from kinematic and supersymmetric considerations. These reduce the calculation of this class of amplitudes to classifying certain polynomials. In the present section we determine these polynomials for the $\alpha^{\prime 3}$ correction to the MHV amplitude for any number of external gluons.

\subsection{Symmetry constraints on the amplitude}

In section 2.4 it was shown that the kinematic and supersymmetric constraints determine that the general $\alpha^{\prime}$-corrected MHV amplitude with $N$ external gluons must have the form

$$
A_{N}\left(-+\cdots-{ }_{j}+\right)=A_{Y M}^{(N)}\left(\sum_{n \geq 0}\left(\alpha^{\prime}\right)^{n} Q_{n}^{(N)}\right)
$$

Here, $N$ is the number of external states in the amplitude. Every $Q_{n}^{(N)}$ is a Lorentz invariant polynomial in the momenta $k_{i}^{\mu}$ of the external particles. To make the prefactor dimensionless, $Q_{n}^{(N)}$ must be homogeneous and have degree $d=2 n$ in the momenta $k_{i}^{\mu}$.

The purpose of this section is to determine how much we can learn about the polynomials $Q_{n}^{(N)}$ using their symmetry properties and the results that are already known in the literature. To this end, let us list some important properties of the $Q^{(N)}$.

- Since we study color-ordered amplitudes (see e.g. [19, 12]), the $Q_{n}^{(N)}$ are cyclically symmetric in the external momenta $k_{i}^{\mu}$.

- From the fact that the three-point functions for massless poles are $\alpha^{\prime}$-independent (see equation (2.2)) it follows that, just like in the Yang-Mills case, $Q_{n}^{(N)}$ must reduce to $Q_{n}^{(N-1)}$ under soft limits:

$$
\left.Q_{n}^{(N)}\right|_{p_{N}^{\mu}=0}=Q_{n}^{(N-1)}
$$


- For the same reason, the behavior under collinear limits generalizes from the YangMills case to the stringy case. That is $Q^{(N)}$ must reduce to $Q^{(N-1)}$ under collinear limits:

$$
\left.Q_{n}^{(N)}\right|_{p_{N}^{\mu}=\alpha p^{\mu}, p_{N-1}^{\mu}=(1-\alpha) p^{\mu}}=\left.Q_{n}^{(N-1)}\right|_{p_{N-1}^{\mu}=p^{\mu}} .
$$

The soft limit is nothing but the special case $\alpha=0$ of the collinear limit, but it is this special case that we are primarily interested in. Note in particular that after we apply a soft limit to a $Q_{n}^{(N)}$, the resulting answer must again be cyclically symmetric in the remaining $p_{i}^{\mu}$. This leads us to the following definition:

Definition. A cyclically reducible polynomial of degree $d$ in $N$ variables is a Lorentz invariant polynomial which is cyclically symmetric in the $N$ variables $p_{i}^{\mu}$, and which, after setting a subset

$$
\left\{p_{i}^{\mu} \mid i \in S \subset\{1, \ldots, N\}\right\}
$$

of the variables to zero, is cyclically symmetric in the remaining variables.

Here, 'cyclically symmetric' does not necessarily mean that the form of the polynomial is the same after a cyclic permutation of the indices. In general, there will be relations between the momenta, such as the momentum conservation condition $\sum p_{i}^{\mu}=0$. By cyclic symmetry, we mean that after a cyclic permutation of the indices, the polynomial comes back to itself up to an expression which vanishes due to these relations.

Our goal will be to find all possible cyclically reducible polynomials of a given degree $d$ in a given number of external particles $N$. Since such polynomials form a vector space, it will be enough to find a basis for this space. As we will see, when $d$ is not too large, these vector spaces have a relatively low dimension which moreover quickly becomes constant when we increase $N$. Our strategy is then to express the known $Q_{n}^{(N)}$ from the literature in terms of this basis. It will turn out that with a smart choice of basis, the coefficients of $Q_{n}^{(N)}$ also become $N$-independent, meaning that we can find a general expression for the $\left(\alpha^{\prime}\right)^{n}$-term in (2.16) which is valid for all $N$. We will carry out this procedure in detail for $n=2$ (for which an answer is known in the literature) and $n=3$ (for which our answer is new).

As a final remark, notice that we do not use collinear limits in the construction of our answer. It would be interesting to reduce the basis of 'allowed' polynomials even further by requiring good behavior under collinear limits. For our current purposes, however, this is unnecessary, since our bases of polynomials are small enough to handle as they are. In fact, we will now be able to use collinear limits as a nontrivial test on our results: after constructing our final answer, we will check explicitly that it behaves as expected under collinear limits.

\subsection{A toy model: scalar variables}

Before we turn to our actual problem, let us develop some intuition by considering a simpler problem. To this end, we replace the momenta $p_{i}^{\mu}$ by scalar variables, which we will denote by $x_{i}$. The problem of finding all cyclically reducible polynomials can now be solved exactly, without the use of a computer. 


\section{Basis polynomials}

To begin with, let us also assume that there are no relations between the $x_{i}-$ we will drop this requirement in a moment. The question as to which polynomials of degree $d$ are cyclically reducible now has a simple answer:

Claim. A basis for the vector space of cyclically reducible polynomials of degree $d$ in $N$ variables consists of the polynomials of the form

$$
\left(a_{1} a_{2} \ldots a_{m}\right) \equiv \sum_{\left(i_{1}, \ldots i_{m}\right)} x_{i_{1}}^{a_{1}} \cdots x_{i_{m}}^{a_{m}}
$$

where $m \leq N, a_{i} \geq 1$ are positive integers with $\sum a_{i}=d$, and the sum is over all cyclically ordered sequences $\left(i_{1}, \ldots i_{m}\right)$ of length $m$. Here, $\left(a_{1} \ldots a_{m}\right)$ that are the same up to a cyclic permutation are considered to be the same basis vector.

By a 'cyclically ordered sequence' $\left(i_{1}, \ldots i_{m}\right)$ we mean that after identifying $N+1$ with 1 , the sequence has 'winding number' +1 . In other words, after an appropriate cyclic permutation, the sequence will be in a strictly increasing order. Thus, for example, $(1,2,3,4,5,6)$, $(1,2,3,5,8,9)$ and $(3,6,7,9,1,2)$ are all cyclically ordered sequences for $d=6$ and $N=9$, but $(1,3,5,6,8,2)$ and $(6,5,4,3,2,1)$ are not. In appendix A.1, we prove the claim that (3.5) forms a basis of cyclically reducible polynomials.

As an example, when $d=4$ and $N=4$, we have the five basis polynomials

$$
(4),(31),(22),(211),(1111)
$$

where for instance

$$
\begin{aligned}
(211)= & x_{1}^{2} x_{2} x_{3}+x_{1}^{2} x_{2} x_{4}+x_{1}^{2} x_{3} x_{4} \\
& +x_{2}^{2} x_{3} x_{4}+x_{2}^{2} x_{3} x_{1}+x_{2}^{2} x_{4} x_{1} \\
& +x_{3}^{2} x_{4} x_{1}+x_{3}^{2} x_{4} x_{2}+x_{3}^{2} x_{1} x_{2} \\
& +x_{4}^{2} x_{1} x_{2}+x_{4}^{2} x_{1} x_{3}+x_{4}^{2} x_{2} x_{3} .
\end{aligned}
$$

Note that if we would have chosen $d=4$ but $N=3$, the polynomial (1111) would vanish since there are simply not enough different variables to write down a term. This is the reason for the constraint $m \leq N$ in our description above. The fact that we need at least $d$ particles to write down the full basis of degree $d$ polynomials will become crucial later on. In general, unless otherwise stated, we will assume that there are enough particles in the amplitude so that the constraint $m \leq N$ does not come into play.

\section{Number of basis polynomials}

To count the number of basis polynomials of a given degree $d$ (assuming as we mentioned above that $N$ is large enough), we need to count the number of sequences of positive integers that add up to $d$, up to cyclic permutations. In mathematics, sequences of integers up to cyclic permutations are called necklaces. The numbers $N_{d}$ of necklaces of arbitrary 
length with positive integer entries that add up to a given $d$ can be found in Sloane's on-line encyclopedia of integer sequences [25] as A008965; it starts off as

$$
1,2,3,5,7,13,19,35,59,107,187,351,631,1181, \ldots \text {. }
$$

In fact, with some smart combinatorics (see appendix A.2) an exact expression for this sequence can be found: it is

$$
N_{d}=-1+\frac{1}{d} \sum_{d_{i} \mid d} \phi\left(d_{i}\right) 2^{d / d_{i}},
$$

where the sum is over all divisors $d_{i}$ of $d$. Here, $\phi\left(d_{i}\right)$ is Euler's totient function, counting the number of integers $k \leq d_{i}$ that are relatively prime to $d_{i}$ :

$$
\phi\left(d_{i}\right)=d_{i} \prod_{p \mid d_{i}}\left(1-p^{-1}\right),
$$

where the product is over all different primes $p$ dividing $d_{i}$. The leading term in this expression tells us that

$$
N_{d} \sim \frac{2^{d}}{d}
$$

is a good estimate for the number of basis polynomials at large $d$.

\section{Momentum conservation}

Now, let us consider the somewhat more realistic problem where there are relations between the generating monomials. In particular, let us study the momentum conservation constraint ${ }^{4}$

$$
\sum x_{i}=0
$$

This constraint means that any two polynomials $P, P^{\prime}$ of degree $d$ represent the same physical quantity if

$$
P=P^{\prime}+\left(\sum x_{i}\right) B
$$

for some polynomial $B$ of degree $d-1$. This defines an equivalence relation on our space of polynomials; a polynomial $P$ is now cyclically reducible if after a cyclic permutation of its variables, it is mapped to a polynomial $P^{\prime}$ in the same equivalence class $[P]$, and if this property is preserved under soft limits.

Clearly, under this new definition, the polynomials (3.5) are still cyclically reducible. Moreover, following the same reasoning as in appendix A.1, it can be shown that they cannot split into a sum $P=P_{1}+P_{2}$ of smaller cyclically reducible polynomials. Since every possible monomial appears in one of the basis polynomials, this means that the basis we constructed before still spans the space of all possible cyclically reducible polynomials. However, it is no longer a basis, since there will be relations between the generating polynomials.

\footnotetext{
${ }^{4}$ In the scalar case, we do not yet include the analog of masslessness constraint, $x_{i}^{2}=0$, since it would oversimplify the problem. Another type of constraint that we will meet in the vector case - the Schouten identity - also has no analog in the scalar model.
} 
To see when this is the case, let us assume that some linear combination of the basis polynomials $P_{j}^{(d)}$ of degree $d$ is zero up to relations:

$$
\sum_{j} c_{j} P_{j}^{(d)}=\left(\sum_{i} x_{i}\right) B^{(d-1)} .
$$

From the permutation symmetry properties of this expression, it is easily seen that this means that $B^{(d-1)}$ must itself be a linear combination of polynomials of the form (3.5) of degree $d-1$. It follows that the set of all relations is spanned by expressions of the form

$$
\left(\sum_{i} x_{i}\right) P_{j}^{(d-1)}
$$

where $P_{j}^{(d-1)}$ form a basis of cyclically reducible polynomials of degree $d-1$. Since the $P_{j}^{(d-1)}$ are linearly independent, so are the relations that they generate.

For clarity, let us again look at an example. For $d=5$, consider the cyclically reducible polynomial

$$
(221)=\sum_{i<j<k} x_{i}^{2} x_{j}^{2} x_{k}
$$

where the sum is over cyclic orderings. Multiplying this with the momentum conservation polynomial

$$
(1)=\sum_{i} x_{i}
$$

we find for $d=6$ the relation

$$
\begin{aligned}
0 \sim(1)(221)= & \left(\sum_{i} x_{i}\right)\left(\sum_{j<k<l} x_{j}^{2} x_{k}^{2} x_{l}\right) \\
= & \sum_{i<j<k} x_{i}^{3} x_{j}^{2} x_{k}+\sum_{i<j<k} x_{i}^{2} x_{j}^{3} x_{k}+\sum_{i<j<k} x_{i}^{2} x_{j}^{2} x_{k}^{2} \\
& +\sum_{i<j<k<l} x_{i}^{2} x_{j} x_{k}^{2} x_{l}+2 \sum_{i<j<k<l} x_{i}^{2} x_{j}^{2} x_{k} x_{l} \\
= & (321)+(231)+(222)+(2121)+2 \cdot(2211) .
\end{aligned}
$$

We see that the algebra is very simple: one just adds the 1 to any of the entries in (221) or inserts a new 1 between two indices. This procedure should be carried out for the entire set of generating cyclically reducible polynomials for $d=5$ to get all relations between the generating cyclically reducible polynomials for $d=6$.

We have thus found that a true basis of cyclically reducible polynomials has size

$$
\hat{N}_{d}=N_{d}-N_{d-1} \sim \frac{2^{d-1}}{d}
$$

a sequence which begins with

$$
0,1,1,2,2,6,6,16,24,48,80,164,280,550, \ldots
$$

where we defined $N_{-1}=1$ to obtain the correct answer $\hat{N}_{1}=0$ for $d=1$. 


\subsection{Vector variables}

As we have seen, constructing a basis of cyclically reducible polynomials and calculating its cardinality is a problem that can be solved explicitly in the scalar variable case. Unfortunately, such an analytic solution is much harder when the variables have a vector index. Here, of course, we require that the resulting polynomial is Lorentz invariant. The naive approach would be to simply construct the same generating polynomials as in the previous section, attach indices to the variables, and contract these indices with metric and epsilon tensors. There are, however, several reasons why this approach is not straightforward; let us mention two important ones.

\section{Failure of the naive basis construction}

The first reason why the naive approach fails is that not all basis vectors can be constructed in this way. To see this consider, for $N=5$ particles, the term

$$
\epsilon_{1234} \equiv \epsilon_{\mu \nu \rho \sigma} p_{1}^{\mu} p_{2}^{\nu} p_{3}^{\rho} p_{4}^{\sigma}
$$

Note that, in principle, we can also define this term for $N=4$ particles, but in that case it would be zero due to momentum conservation. If we would naively construct our basis of cyclically reducible polynomials in the same way as in the previous section, we would also include a term $\epsilon_{1235}$ in the polynomial that contains $\epsilon_{1234}$. But from momentum conservation for five particles, we see that

$$
\epsilon_{1234}+\epsilon_{1235}=0
$$

In this way, all terms in the naively constructed polynomial cancel pairwise, and the entire

polynomial vanishes. Nevertheless, there is a cyclically reducible polynomial consisting of terms of this type; it can be written in a manifestly cyclically symmetric way as

$$
\epsilon_{1234}+\epsilon_{2345}+\epsilon_{3451}+\epsilon_{4512}+\epsilon_{5123}
$$

or, since all of these terms are equal due to momentum conservation and antisymmetry, simply as $\epsilon_{1234}$. Thus, we see that our naive construction fails. The (vanishing) polynomial constructed from $\epsilon_{1234}$ using our naive method is of course still cyclically reducible, but it can now be written as a sum of smaller (nonvanishing but mutually cancelling) cyclically reducible polynomials. The reason our proof in appendix A.1 fails in this case is that it relies on the fact that monomials do not vanish when we take a soft limit in an index that does not appear explicitly.

\section{Relations between relations}

Another technical difficulty in the case of vector variables is the fact that the relations one constructs are no longer independent. To see this, let us introduce the notation

$$
s_{i j}=g_{\mu \nu} p_{i}^{\mu} p_{j}^{\nu}
$$


These variables satisfy $s_{i j}=s_{j i}$ and, by masslessness, $s_{i i}=0$ (no summation), so for $N$ particles there are $N(N-1) / 2$ of them. Momentum conservation now leads to $N$ relations between the $s$-variables of the form

$$
\sum_{i} s_{i j}=0, \quad 1 \leq j \leq N
$$

If we would be considering $d=4$, this would then give $N^{2}(N-1) / 2$ relations

$$
r_{j k l} \equiv \sum_{i} s_{i j} s_{k l}=0, \quad 1 \leq j \leq N, \quad 1 \leq k<l \leq N
$$

Here, the restriction $k<l$ is only not to overcount, but we define $r_{j k l}$ for $k>l$ similarly. If we now sum over all $k$ at a given $j$ and $l$ we obtain

$$
\sum_{k} r_{j k l}=\sum_{i, k} s_{i j} s_{k l}=\sum_{i} r_{l i j}
$$

Thus, for each choice of $(j, l)$, we have found a linear relation between a subset of the relations. We need to correct for such 'relations between relations' when counting the number of linearly independent basis polynomials.

\section{Generic counting of polynomials}

Even though we have not found a simple closed formula for the number of cyclically reducible polynomials of given degree in the vector variable case, let us mention that we can at least find a simple formula for the number of contractions of $2 n$ four-dimensional Lorentz indices modulo Schouten identities. As explained in appendix A.4 this is given by the $n$ 'th Catalan's number squared. Note that this number grows exponentially for large $n$ as

$$
C_{n}=\left(c_{n}\right)^{2} \sim \frac{2^{4 n}}{\pi n^{3}} \quad \text { for } n \rightarrow \infty .
$$

Although the explicit forms of the contractions themselves can also straightforwardly be worked out, they will not be needed in this paper.

\section{Computer approach}

Taking all of the subtleties mentioned above into account in the analytic approach is a very tedious excercise, though probably not impossible. We will leave this for future work though, and for the moment let a computer do the hard work of counting Lorentz invariant cyclically reducible basis polynomials for vector variables. From the scalar variable example, we expect that the number of basis polynomials we will find is constant above a given number of particles, which will be of the order $N \sim d$. Moreover, we expect the basis to be expressible in a form which is independent of the number of particles, as in our $\left(a_{1} \ldots a_{m}\right)$ notation above. We can therefore try to solve the problem for given $d$ and small $N$ using a computer, and then conjecture that once the answer stabilizes in terms of $N$, we have found our general answer. It is this approach that we now turn to. 


\subsection{Methodology}

We have implemented our computer construction of a basis of cyclically reducible polynomials of given degree using Mathematica. For definiteness, let us describe the case of degree six; the generalization to other degrees is straightforward.

For $d=6$, there are two ways to contract the Lorentz indices of the six momentum vectors appearing in a given term: either by three metric tensors or by one $\epsilon$ - and one metric tensor. In the notation used above, the first contraction will lead to terms of the schematic form sss; the second contraction to terms of the schematic form $\epsilon s$. Note that there will be no relations mixing terms of these different forms, so we can construct a basis of cyclically reducible polynomials for each form separately, and then combine the answers in the end. Here, for illustrative purposes, we will discuss the $\epsilon s$ polynomials - the case of sss polynomials is treated in a completely analogous manner.

Suppose now that we have $N$ external particles. We work by induction, so we assume that we already know a basis of cyclically reducible polynomials ${ }^{5}$ for $N-1$ particles. In this particular example we can start the induction at $N=5$, since for $N=4$ all polynomials that one can write down are zero (and hence cyclically reducible) because the $\epsilon$-factor vanishes by symmetry and momentum conservation relations.

\section{Variables, monomials, polynomials}

First, we define the following variables:

$$
s_{i j}=g_{\mu \nu} p_{i}^{\mu} p_{j}^{\nu}, \quad 1 \leq i<j \leq N
$$

and

$$
\epsilon_{i j k l}=\epsilon_{\mu \nu \rho \sigma} p_{i}^{\mu} p_{j}^{\nu} p_{k}^{\rho} p_{l}^{\sigma}, \quad 1 \leq i<j<k<l \leq N .
$$

Below, we will often encounter $s_{i j}$ and $\epsilon_{i j k l}$ where the indices are not ordered in the above way; we take these expressions to be defined in the obvious way: $s_{j i}=s_{i j}, \epsilon_{j i k l}=-\epsilon_{i j k l}$, etc. Next, we define a set of monomials

$$
m_{i}, \quad 1 \leq i \leq M_{N}
$$

consisting of products of one $\epsilon$ - and one $s$-variable. Here

$$
M_{N}=\left(\begin{array}{c}
N \\
2
\end{array}\right)\left(\begin{array}{c}
N \\
4
\end{array}\right)
$$

is the product of the number of $\epsilon$ - and $s$-variables. Finally, we define $\mathcal{P}_{N}$ to be the $M_{N^{-}}$ dimensional vector space generated by the $m_{i}$. We will thus represent polynomials $q=q^{i} m_{i}$ as vectors $q^{i}$ consisting of $M_{N}$ numbers.

\footnotetext{
${ }^{5}$ Actually, as we will see below, it is technically more convenient to store a basis of polynomials perpendicular to the space of cyclically reducible ones.
} 


\section{Relations}

Define the subspace $\mathcal{R}_{N}$ to be the space generated by all relations between the $m_{i}$. These relations arise as follows:

1. Relations from momentum conservation on $s$-variables. These relations take the form

$$
\epsilon_{i j k l}\left(\sum_{x \neq m} s_{m x}\right)=0
$$

for all values of $i, j, k, l, m$ with $i<j<k<l$.

2. Relations from momentum conservation on $\epsilon$-variables. These relations take the form

$$
\left(\sum_{x \notin\{i, j, k\}} \epsilon_{x i j k}\right) s_{l m}=0,
$$

for all values of $i, j, k, l, m$ with $i<j<k$ and $l<m$.

3. Schouten identities: an antisymmetrized five-index tensor in four dimensions must vanish identically, meaning that

$$
\epsilon_{i j k l} s_{m n}+\epsilon_{j k l m} s_{i n}+\epsilon_{k l m i} s_{j n}+\epsilon_{l m i j} s_{k n}+\epsilon_{m i j k} s_{l n}=0,
$$

for all values of $i, j, k, l, m, n$ with $i<j<k<l<m$.

In this way, we find $R_{N}$ relations of the form $r_{\alpha}{ }^{j} m_{j}=0$, where $\alpha$ labels the different relations. We will think of $r$ as an $R_{N} \times M_{N}$ matrix in what follows. The relations will not all be independent (that is, the rank of $r$ will be less than $R_{N}$ ), but all that matters to us is that we have found a set that spans $\mathcal{R}_{N}$.

In what follows, it turns out to be useful to equip $\mathcal{P}_{N}$ with a metric, which we take to be the identity matrix in the basis provided by the $m_{i}$. When we have a matrix such as $r$ whose rows span a certain space, we will denote a matrix whose row vectors span the perpendicular space by $\left(r^{\perp}\right)$ :

$$
\forall \alpha, \beta: \quad\left(r^{\perp}\right)_{\alpha l} r_{\beta}^{l}=0 .
$$

This construction is implemented in Mathematica by the command Nullspace.

\section{Operators}

Next, we construct two operators:

- An $M_{N} \times M_{N}$ matrix $c^{i}{ }_{j}$ that represents the cyclic permutations of the external particles on $\mathcal{P}_{N}$. For example, $\epsilon_{1234} s_{12} \rightarrow \epsilon_{2345} s_{23}$ under a cyclic permutation, which can be written as $m_{a} \rightarrow m_{b}$ for some $a$ and $b$. Thus, $c^{j}{ }_{a}=1$ for $j=b$ and $c^{j}{ }_{a}=0$ for $j \neq b$.

- An $M_{N-1} \times M_{N}$ matrix $\pi^{I}{ }_{j}$ that projects onto the polynomials that survive when we set the momentum $p_{N}=0$. In our implementation, we order the variables in such a way that the first $M_{N-1}$ monomials $m_{i}$ are the ones that do not involve $p_{N}$, so the matrix $\pi$ consists of an $M_{N-1} \times M_{N-1}$ identity matrix adjoined by $M_{N}-M_{N-1}$ columns of zeroes. 


\section{Cyclically reducible polynomials}

We will denote the space of all cyclically reducible polynomials by $\mathcal{Z}_{N}$. That is, $\mathcal{Z}_{N}$ consists of all $q^{j}$ such that

$$
(1-c)^{i}{ }_{j} q^{j} \in \mathcal{R}_{N}, \quad \pi^{I}{ }_{j} q^{j} \in \mathcal{Z}_{N-1} .
$$

The first equation imposes cyclic symmetry (up to relations); the second one the reduction to a cyclically reducible polynomial of one particle less. We rewrite the first equation as

$$
\left(r^{\perp}\right)_{\alpha i}(1-c)^{i}{ }_{j} q^{j}=0
$$

Thus, we are looking for all vectors perpendicular to the rows of the matrix $r^{\perp} \cdot(1-c)$. Similarly, we can write the second equation in (3.37) as the search for all $q$ that satisfy

$$
z_{N-1}^{\perp} \cdot \pi \cdot q=0
$$

where $z_{N-1}$ is a matrix whose rows span $\mathcal{Z}_{N-1}$. It follows that in order to construct $z_{N}^{\perp}$, all we need to do is combine the rows of $r^{\perp} \cdot(1-c)$ and $z_{N-1}^{\perp} \cdot \pi$ into a single matrix. We could of course construct $z_{N}$ from this by looking for a basis of perpendicular vectors, but as we saw it is $z_{N}^{\perp}$ which we will need at the next step of the induction, so we do not need to do so.

Finally, if we want to know the number of cyclically reducible polynomials up to relations, it is $M_{N}-\operatorname{rank}\left(z_{N}^{\perp}\right)$. Therefore, the 'true' number of cyclically reducible polynomials (the ones that are not zero by relations) is

$$
Z_{N}^{(\epsilon s)}=M_{N}-\operatorname{rank}\left(z_{N}^{\perp}\right)-R_{N},
$$

where we included a superscript $(\epsilon s)$ to remind the reader that, in the example we treated in this section, we would still only have calculated the number of basis polynomials containing terms of the form $\epsilon s$. To complete the calculation, we then have to run the analogous code for polynomials of the form sss, to find the cardinality of the total basis:

$$
Z_{N}=Z_{N}^{(\epsilon s)}+Z_{N}^{(s s s)}
$$

For different degrees, we of course have similar expressions.

As a first check on our algorithms, we have checked that for scalar variables, up to degree 8 , the analytic results of section 3.2 are reproduced.

\subsection{Results}

In this section, we collect the results of our computer calculations, and comment on how much further this approach can be taken.

\section{The $\left(\alpha^{\prime}\right)^{2}$ term}

For degree $d=4$, corresponding to the $\left(\alpha^{\prime}\right)^{2}$ term in the string expansion, the exact answer for the amplitude was found by Stieberger and Taylor in [0]. Therefore, this is 
a good testing ground for our algorithms. We find that in this case the number $Z_{N}$ of cyclically reducible polynomials for $N$ particles is

$$
Z_{1}=Z_{2}=Z_{3}=0, \quad Z_{4}=2, \quad Z_{N \geq 5}=3 .
$$

To check that the number of cyclically reducible basis polynomials indeed becomes constant, we have checked that $Z_{N}=3$ for $5 \leq N \leq 11$. It is worth noting that, contrary to the scalar case, constancy does not set in at $N=d$ but at $N=d+1$. The reason for this is that at $N=4$, the $\epsilon$-variables still vanish due to momentum conservation.

An explicit $N$-independent realization of the three basis polynomials is

$$
P_{1}=\left[s_{12} s_{34}\right] \quad, \quad P_{2}=\left[s_{13} s_{24}\right] \quad, \quad P_{3}=\sum_{i<j<k<l} \epsilon_{i j k l} .
$$

In the first two polynomials, the square brackets mean that we sum over all cyclically equivalent sets of indices, just like we did in the scalar variable case. As we mentioned before, for the $\epsilon_{i j k l}$ terms this cannot be done, which is why we use a different summation.

We have checked that up to 11 particles, the Stieberger-Taylor answer can be expressed in terms of these polynomials as

$$
Q_{2}^{(N)}=-\frac{\pi^{2}}{12}\left(P_{1}-P_{2}+4 i P_{3}\right)
$$

This is a significant simplification compared to the general formula given in equation (74) of [7]. In fact, the result can be written even more compactly as

$$
Q_{2}^{(N)}=-\frac{\pi^{2}}{6} \sum_{i<j<k<l}\langle i j\rangle[j k]\langle k l\rangle[l i],
$$

where we have used standard spinor notation.

\section{The $\left(\alpha^{\prime}\right)^{3}$ term}

After checking our algorithms with known results, we are now ready to produce some new ones. The general answer for the $\left(\alpha^{\prime}\right)^{3}$ term in the MHV amplitude is not known in the literature. However, the full amplitudes for 4, 5, 6 [7] and 7 [8] external gluons is known from the work of Stieberger and Taylor, so we can extract the $\left(\alpha^{\prime}\right)^{3}$ coefficient from their expressions ${ }^{6}$.

Using our computer program, we have found that the number $Z_{N}$ of cyclically reducible basis polynomials up to relations for $d=6$ and $N$ particles is

$$
Z_{1}=Z_{2}=Z_{3}=0, \quad Z_{4}=2, \quad Z_{5}=6, \quad Z_{N \geq 6}=13 .
$$

Note that here, contrary to the $\left(\alpha^{\prime}\right)^{2}$ case, constancy of $Z_{N}$ sets in at the 'naive' value of $N=6$. We have checked the constancy of $Z_{N}$ for up to 8 particles and for the terms involving $\epsilon$-tensors up to 9 particles.

\footnotetext{
${ }^{6}$ In fact, since their 7-particle amplitude is written in terms of complicated Euler integrals, it is quite difficult to extract an explicit $\alpha^{\prime}$ expansion from it, and we have not succeeded in doing so. Fortunately, as we will see, for our purposes it is sufficient to know the six-particle amplitude.
} 
Since we have the full set of basis polynomials at $N=6$, we can expand the answer of Stieberger and Taylor in this basis. We conjecture that this in fact gives the general $\left(\alpha^{\prime}\right)^{3}$ answer for any $N$, as was the case for $\left(\alpha^{\prime}\right)^{2}$. Because of the large number of relations, there are many equivalent ways to write the answer; one such way is

$$
\begin{aligned}
Q_{3}^{(N)}= & \frac{\zeta(3)}{24}\left(42\left[s_{12} s_{34} s_{56}\right]+18\left[s_{13} s_{24} s_{56}\right]-9\left[s_{13} s_{23} s_{56}\right]+9\left[s_{13} s_{25} s_{46}\right]-3\left[s_{14} s_{25} s_{36}\right]\right. \\
& \left.+36\left[s_{12} s_{15} s_{34}\right]-\left[s_{12} s_{12} s_{12}\right]+96 i\left[\epsilon_{1234} s_{56}\right]+24 i\left[\epsilon_{1234} s_{45}\right]-24 i\left[\epsilon_{1234} s_{35}\right]\right),(3.47)
\end{aligned}
$$

where once again, the square bracket notation means that we should sum over all index sets with the same cyclic ordering. Note that this expression contains only ten terms; the coefficients of the other three basis vectors are vanishing.

To check that this expression gives the answer for general $N$, we have checked that also for 4 and 5 particles, it reproduces the Stieberger-Taylor answer. It would be an even better check to see if this answer also reproduced the seven-particle answer (where we have 13 coefficients to compare, instead of the 2 and 6 for $N=4,5$ ), but due to the technicalities mentioned in the last footnote, we have not been able to carry out this calculation. Instead, we will now turn to a different and quite nontrivial check of (3.47): we will test if it behaves well under collinear limits.

\section{Collinear limits}

As we mentioned at the beginning of this section, the polynomial $Q_{3}^{(N)}$ should satisfy

$$
\left.Q_{3}^{(N)}\right|_{k_{N}^{\mu}=\alpha p^{\mu}, p_{N-1}^{\mu}=(1-\alpha) p^{\mu}}=\left.Q_{3}^{(N-1)}\right|_{p_{N-1}^{\mu}=p^{\mu}} .
$$

Let us now check explicitly that this is the case. To this end, we rewrite all terms in (3.47) in the collinear limit. For example, we have

$$
\begin{aligned}
{\left[s_{12} s_{34} s_{56}\right] \rightarrow } & \left(s_{12} s_{34} s_{56}\right) \\
& +\alpha\left(s_{12} s_{34} s_{5 x}\right)+\text { cyclic } \\
& +(1-\alpha)\left(s_{12} s_{34} s_{5 x}\right)+\text { cyclic } \\
& +\left(\alpha-\alpha^{2}\right)\left(s_{23} s_{4 x} s_{x 1}\right)+\left(\alpha-\alpha^{2}\right)\left(s_{4 x} s_{x 1} s_{23}\right)+\left(\alpha-\alpha^{2}\right)\left(s_{x 1} s_{23} s_{4 x}\right) .
\end{aligned}
$$

Here, on the right hand side, all expressions are for $N-1$ particles. The index $x$ stands for the index with value $N-1$; the round brackets mean that we sum over all index sets that have the same cyclic structure as the term within brackets, where the $x$ always remains $N-1$. The first line on the right hand side comes from the terms on the left hand side where no index equals $N$ or $N-1$. The second line comes from the terms where one index equals $N$ and no index equals $N-1$; the third line comes from the converse. The fourth line comes from the terms where both an index $N$ and an index $N-1$ are present. When adding the terms, we see that the $\alpha$-dependent terms in the first three lines cancel, and the constant terms add up to the required term of one particle less. The nontrivial part of the answer comes from the fourth line, and we find

$$
\left[s_{12} s_{34} s_{56}\right]_{N} \rightarrow\left[s_{12} s_{34} s_{56}\right]_{N-1}+3\left(\alpha-\alpha^{2}\right)\left(s_{23} s_{1 x} s_{4 x}\right) .
$$


Carrying out this procedure for every term in (3.47), we find

$$
\begin{aligned}
Q_{3}^{(N)} \rightarrow & Q_{3}^{(N-1)}+\frac{\zeta(3)}{12}\left(\alpha-\alpha^{2}\right) \times \\
& \left\{18\left(s_{12} s_{1 x} s_{3 x}\right)+18\left(s_{13} s_{2 x} s_{4 x}\right)+6\left(s_{1 x} s_{1 x} s_{1 x}\right)\right. \\
+ & 18\left(s_{23} s_{1 x} s_{3 x}\right)+36\left(s_{23} s_{1 x} s_{4 x}\right)+18\left(s_{24} s_{1 x} s_{3 x}\right) \\
& \left.-12 i\left(\epsilon_{x 123} s_{2 x}\right)+24 i\left(\epsilon_{234 x} s_{1 x}\right)-24 i\left(\epsilon_{123 x} s_{3 x}\right)-24 i\left(\epsilon_{134 x} s_{2 x}\right)-48 i\left(\epsilon_{123 x} s_{4 x}\right)\right\} .
\end{aligned}
$$

In principle, there could have been terms cubic in $\alpha$ in this expression, coming from terms where three $s$-indices become $N$ or $N-1$, but it turns out that these terms cancel. Moreover, the terms linear and quadratic in $\alpha$ turn out to multiply the same polynomial up to a sign. At first sight, however, this polynomial does not seem to vanish. Fortunately, one can show using momentum conservation and the Schouten identities, that it actually does vanish. We give the details of this calculation in appendix A.3. Inserting this result, we find that

$$
Q_{3}^{(N)} \rightarrow Q_{3}^{(N-1)}
$$

so the answer (3.47) indeed has the correct behavior under collinear limits, as we wanted to show.

\section{Order $\left(\alpha^{\prime}\right)^{4}$ and beyond?}

Using our current Mathematica implementation, it seems difficult to continue our computer calculations beyond $d=6$. For example, the calculation for $d=6$ and $N=8$ takes roughly an hour on an average desktop. On the other hand, using faster computers and a more efficient programming language, it might be possible to carry out the $\left(\alpha^{\prime}\right)^{4}$ calculation up to 8 particles, which is the naive number of particles for which we should find the full basis of cyclically reducible polynomials.

However, this calculation will only give us the cardinality of the basis for the space of cyclically reducible polynomials, which, if the $2^{d} / d$ behavior we found in the scalar case is a good measure, we expect to be of order 40 or so. This number is not too large, and the construction of an actual basis should not be too difficult either, but the problem is that after that, we do not have anything to compare to: the eight-particle MHV amplitude has only been calculated up to order $\left(\alpha^{\prime}\right)^{2}$. Thus, it would not be possible to express the known answer in our basis and find a conjecture for the full $N$-independent answer. ${ }^{7}$ The best one could do at the moment would be to use the seven-particle answer at $\left(\alpha^{\prime}\right)^{4}$, which would fix only part of the coefficients of the full answer.

Needless to say, these problems only get more severe at even higher orders. Even if one could find smarter algorithms to construct a basis for cyclically reducible polynomials of degree 10, we would need at least the 10-particle amplitude up to order $\left(\alpha^{\prime}\right)^{5}$ to express the full answer in terms of this basis.

\footnotetext{
${ }^{7}$ Possibly, the techniques that were developed quite recently in 9 could be helpful in finding explicit expressions for higher-order corrections to higher point amplitudes.
} 
One solution to this problem might be to study polynomials that have the correct reduction properties under collinear limits as well as soft limits. In our $\left(\alpha^{\prime}\right)^{3}$ calculation, we have seen that requiring good collinear limits relates a polynomial of the form $\left[s_{12} s_{34} s_{56}\right]$ to polynomials which have several indices that are equal. The latter polynomials can be defined for a smaller number of particles. Thus, using a basis of 'collinear cyclically reducible polynomials' of degree $d=8$, we might find that this basis already reaches its maximum size for some number $N<8$ of particles. If this is the case, the general $\left(\alpha^{\prime}\right)^{4}$ answer can be constructed using our methods above and the results that are known in the literature.

However, also this approach will have a limited applicability - it appears unlikely that it will work for degrees above 4 . Therefore, it seems that ultimately, one should go back to the analytic approach. In this respect, the answer (3.45) gives hope due to its simplicity. It would be nice to express (3.47) in an equally simple way in spinor notation, so one can find a pattern that might extend to higher orders. Unfortunately, the most naive generalization,

$$
\sum_{i<j<k<l<m<n}\langle i j\rangle[j k]\langle k l\rangle[l m]\langle m n\rangle[n i]
$$

though it turns out to be cyclically reducible, cannot be the answer we are looking for: it vanishes after a single soft limit. We have found some expressions for $Q_{3}^{(N)}$ in terms of spinor notations that do work, but they are not any nicer than (3.47), and do not provide any intuition on how to proceed to higher degree.

\section{CSW rules for the Abelian DBI action}

The previous section focused on solving the constraints on the gluon scattering amplitude of the superstring which follow from supersymmetry and kinematics. As emphasized before, one of the reasons one is interested in these amplitudes is to derive an expression for the string effective action from them. This effective action can then be taken off-shell to further study the behavior of the string theory. However, the effective action may be obtained by other methods, and given an expression for the effective action, one may conversely ask what amplitudes this action generates. Especially in the case of amplitudes of strings ending on a single D-brane quite a lot is known about the effective action, in particular because in this case one has a reliable derivative expansion. This leads for instance to the well-known Dirac-Born-Infeld action, see e.g. [17] to which the reader is also referred for general background information. Motivated by a desire to obtain CSW-style perturbation theory, we study in this section the Abelian Dirac-Born-Infeld effective action reduced to four dimensions and its tree level scattering amplitudes.

\subsection{Dirac-Born-Infeld in four dimensions}

In general, the string effective action for Abelian fields can have terms of the form

$$
\mathcal{L}_{\text {deriv }} \sim \alpha^{\prime m} \partial^{n} F^{p}
$$


Simple dimensional analysis restricts $p=(m+2-n / 2)$, and it is also known that $p$ cannot be odd because of worldsheet parity (we will shortly encounter a second reason why this is true in four dimensions). The corrections to ordinary electrodynamics are known at the level of the action for

1. terms without derivatives $(n=0)$

2. terms with four fields [26] $(p=4)$

3. (up to) four derivative terms [27] $(n=4)$

The leading derivative corrections are given by the DBI action, while the second simply follow from the known Veneziano amplitude (2.3) for four photons. Although interesting, we will not consider the third series of terms in this paper. The DBI action reads

$$
S_{\mathrm{DBI}}=-1+\frac{1}{\pi^{2} g_{s} \alpha^{\prime}} \int d^{10} x \sqrt{-\operatorname{det}\left(\eta_{\mu \nu}+\pi \alpha^{\prime} F_{\mu \nu}\right)},
$$

which can be derived in several ways [17. In this section, we will put $g_{s}=1$ and drop the irrelevant ' -1 ' term ${ }^{8}$. We will adhere to the convention that

$$
Z[J]=\int e^{i S},
$$

but note that in the $n$-point amplitudes given below we omit an overall normalization factor of $i$.

Although the above (part of the) effective action can be studied in any number of dimensions in principle, here only the dimensional reduction to four dimensions will be considered. In other words, in terms of the scattering amplitudes 4 directions will be selected and all momenta and polarization vectors of the external fields will be embedded in these dimensions. With this choice it is obvious that in practice one can simply ignore all off-dimensional parts of the action and replace $10 \rightarrow 4$ by ordinary dimensional reduction. Since in four dimensions it is known that the determinant in (4.2) is a Lorentz invariant of maximal order 4 in the field strength, one can write down a generic ansatz for the determinant in terms of these. Both the ansatz as well as the determinant can be evaluated explicitly by simply writing $\eta_{\mu \nu}$ and $F_{\mu \nu}$ as matrices ${ }^{9}$. Comparing the expressions gives after some experimentation

$$
-\operatorname{det}\left(\eta_{\mu \nu}+\pi \alpha^{\prime} F_{\mu \nu}\right)=1+\frac{\pi^{2} \alpha^{\prime 2}}{2} F_{\mu \nu} F^{\mu \nu}+\frac{\pi^{4} \alpha^{\prime 4}}{16}\left(F_{\mu \nu} \tilde{F}^{\mu \nu}\right)^{2} .
$$

In the following we will find it useful to write the DBI action in terms of selfdual and anti-selfdual field strengths. In four dimensions the field strength tensor can be decomposed uniquely in terms of a selfdual and an anti-selfdual field strength,

$$
F_{\mu \nu}=F_{\mu \nu}^{+}+F_{\mu \nu}^{-}
$$

\footnotetext{
${ }^{8}$ The explicit dependence on $\pi$ will be useful to make some comments about the 'transcendentality' of the scattering amplitudes.

${ }^{9}$ We define $\tilde{F}_{\mu \nu} \equiv \frac{\mathrm{i}}{2} \epsilon_{\mu \nu}^{\rho \sigma} F_{\rho \sigma}$
} 
which in turn have a natural expression in terms of spinor variables,

$$
F_{\mu \nu} \leftrightarrow F_{\alpha \beta \dot{\alpha} \dot{\beta}}=\epsilon_{\alpha \beta} F_{\dot{\alpha} \dot{\beta}}^{+}+\epsilon_{\dot{\alpha} \dot{\beta}} F_{\alpha \beta}^{-} .
$$

Note that in our conventions,

$$
\tilde{F}_{\alpha \beta \dot{\alpha} \dot{\beta}}=\epsilon_{\alpha \beta} F_{\dot{\alpha} \dot{\beta}}^{+}-\epsilon_{\dot{\alpha} \dot{\beta}} F_{\alpha \beta}^{-} \text {. }
$$

This is of course nothing but the observation that the tensor $F_{\alpha \beta \dot{\alpha} \dot{\beta}}$ must be antisymmetric under exchange of $(\alpha \dot{\alpha}) \leftrightarrow(\beta \dot{\beta})$, so it can be decomposed in terms that are anti-symmetric in either the dotted or undotted indices. As there is only one antisymmetric $2 \times 2$ matrix, these terms must be proportional to the $2 \times 2 \epsilon$ symbol, and the above conclusion follows for a choice of normalization contained in the equation. In terms of the spinor field strengths the action (4.2) reads

$$
S_{\mathrm{DBI}}=\frac{1}{\pi^{2} \alpha^{\prime 2}} \int d^{4} x \sqrt{\left(1+\frac{\pi^{2} \alpha^{\prime 2}}{8}\left(F_{+}^{2}+F_{-}^{2}\right)\right)^{2}-\frac{\pi^{4} \alpha^{\prime 4}}{16} F_{+}^{2} F_{-}^{2}} .
$$

This can now be expanded in powers of $\alpha^{\prime}$, either symmetrically in the field strengths

$$
\begin{aligned}
\mathcal{L}_{\mathrm{DBI}}=\frac{1}{4} F_{+}^{2}-\frac{\pi^{2} \alpha^{\prime 2}}{32}\left(F_{-}^{2} F_{+}^{2}\right)+ & \frac{\pi^{4} \alpha^{\prime 4}}{256}\left(F_{-}^{4} F_{+}^{2}+F_{+}^{4} F_{-}^{2}\right) \\
& -\frac{\pi^{6} \alpha^{\prime 6}}{2048}\left(F_{-}^{6} F_{+}^{2}+3 F_{-}^{4} F_{+}^{4}+F_{+}^{6} F_{-}^{2}\right)+\mathcal{O}\left(\alpha^{\prime 8}\right),
\end{aligned}
$$

or asymmetrically

$$
\mathcal{L}_{\mathrm{DBI}}=\frac{1}{4} F_{+}^{2}-\frac{\pi^{2} \alpha^{\prime 2}}{32}\left(\frac{F_{-}^{2} F_{+}^{2}}{1+\frac{1}{8} \pi^{2} \alpha^{\prime 2} F_{-}^{2}}\right)+\frac{\pi^{4} \alpha^{\prime 4}}{256}\left(\frac{F_{-}^{2}\left(F_{+}^{2}\right)^{2}}{\left(1+\frac{1}{8} \pi^{2} \alpha^{\prime 2} F_{-}^{2}\right)^{3}}\right)+\mathcal{O}\left(F_{+}^{6}\right)
$$

where we have furthermore added the topological density $\left(\frac{1}{4} F_{\mu \nu} \tilde{F}^{\mu \nu}=\frac{1}{8}\left(F_{+}^{2}-F_{-}^{2}\right)\right)$. As we will see below, the last form (4.10) is more geared towards deriving MHV amplitudes.

\subsection{Deriving scattering amplitudes from the DBI action}

Writing the DBI action in terms of selfdual and anti-selfdual fields is advantageous because for on-shell photon fields of definite helicity, $A^{+}$and $A^{-}$, these field strengths simplify. The polarization vectors of these on-shell fields are given in our conventions ${ }^{10}$ by

$$
\epsilon_{\alpha \dot{\alpha}}^{+}=\sqrt{2} \frac{\eta_{\alpha} p_{\dot{\alpha}}}{[\eta p]}, \quad \epsilon_{\alpha \dot{\alpha}}^{-}=\sqrt{2} \frac{\eta_{\dot{\alpha}} p_{\alpha}}{\langle\eta p\rangle} .
$$

Therefore we have in spinor notation

$$
\begin{aligned}
& F_{\dot{\alpha} \dot{\beta}}^{+}\left[A^{+}\right]=i \sqrt{2} p_{\dot{\alpha}} p_{\dot{\beta}}, \quad F_{\dot{\alpha} \dot{\beta}}^{+}\left[A^{-}\right]=0 \\
& F_{\alpha \beta}^{-}\left[A^{-}\right]=i \sqrt{2} p_{\alpha} p_{\beta}, \quad F_{\alpha \beta}^{-}\left[A^{+}\right]=0 .
\end{aligned}
$$

\footnotetext{
${ }^{10}$ Our spinor conventions are such that $p_{\alpha \dot{\alpha}}=\sigma_{\alpha \dot{\alpha}}^{\mu} p_{\mu}$, from which $g_{\mu \nu} \leftrightarrow 2 \epsilon_{\alpha \beta} \epsilon_{\dot{\alpha} \dot{\beta}}$. Dotted spinors are called holomorphic. Furthermore $\epsilon_{\alpha \beta} \epsilon^{\beta \gamma}=\delta_{\alpha}^{\gamma}$, and we have $\langle a b\rangle=a^{\dot{\alpha}} b_{\dot{\alpha}}=a^{\dot{\alpha}} b^{\dot{\beta}} \epsilon_{\dot{\beta} \dot{\alpha}},[a b]=a_{\alpha} b^{\alpha}=a^{\alpha} b^{\beta} \epsilon_{\alpha \beta}$. These conventions largely follow 24].
} 
Note that the $(+,-)$ superscripts on the fields strengths only correspond to the helicity quantum number on-shell. This will be very important when considering larger Feynman diagrams. The above equations imply that in a given Feynman diagram derived from the four-dimensional DBI action the contractions between vertices and external states are in fact very simple. For these 'external line' factors field strength superscripts immediately translate into helicity superscripts. For the four-point scattering amplitude for instance, which is controlled by just one vertex from (4.9), it follows therefore immediately that the amplitude derived from the DBI action reads

$$
A_{4}^{\mathrm{DBI}}\left(1^{+} 2^{+} 3^{-} 4^{-}\right)=-\frac{\pi^{2} \alpha^{\prime 2}}{2}\langle 12\rangle^{2}[34]^{2},
$$

and that the following amplitudes vanish

$$
A_{4}^{\mathrm{DBI}}\left(1^{+} 2^{+} 3^{+} 4^{+}\right)=A_{4}^{\mathrm{DBI}}\left(1^{-} 2^{-} 3^{-} 4^{-}\right)=A_{4}^{\mathrm{DBI}}\left(1^{-} 2^{+} 3^{+} 4^{+}\right)=A_{4}^{\mathrm{DBI}}\left(1^{+} 2^{-} 3^{-} 4^{-}\right)=0 .
$$

The 'DBI' superscript is written here and elsewhere in this section to remind the reader that since these amplitudes were derived from the DBI action, they are only the lowest order correction in $\alpha^{\prime}$ to the full superstring amplitudes. Note that the MHV amplitude in (4.13) is the full amplitude, so it does not include a suppressed sum over permutations of external particles.

For more particles we need to take into account the 'interior' of the Feynman graphs: contractions between the photon fields within the field strength tensors in the different vertices. Note that for this the $(+,-)$ superscripts do not correspond to the helicity quantum number. As can be checked explicitly in Feynman-'t Hooft gauge for instance, these contractions are

$$
\begin{aligned}
& \ldots F_{\dot{\alpha} \dot{\beta}}^{+}:: F_{\alpha \beta}^{-} \ldots=-i\left(\frac{p_{\alpha \dot{\alpha}} p_{\beta \dot{\beta}}+p_{\beta \dot{\alpha}} p_{\alpha \dot{\beta}}}{p^{2}}\right) \\
& \ldots F_{\dot{\alpha} \dot{\beta}}^{+}:: F_{\dot{\delta} \dot{\gamma}}^{+} \ldots=i\left(\epsilon_{\dot{\alpha} \dot{\delta}} \epsilon_{\dot{\beta} \dot{\gamma}}+\epsilon_{\dot{\beta} \dot{\delta}} \epsilon_{\dot{\alpha} \dot{\gamma}}\right) \\
& \ldots F_{\alpha \beta}^{-}:: F_{\delta \gamma}^{-} \ldots=i\left(\epsilon_{\alpha \delta} \epsilon_{\beta \gamma}+\epsilon_{\alpha \gamma} \epsilon_{\beta \delta}\right) .
\end{aligned}
$$

Hence, the contraction between photon field strengths of the same type is nonzero and local in space-time as it simply does not depend on the momentum. This is the result of a cancellation of the propagator pole through a numerator factor. It is only non-local between field strength tensors of opposite type. Note that this 'local' property of the contraction does not depend on any on-shell conditions: the cancellation occurs off-shell. A similar phenomenon was discussed in the context of effective Higgs-gluon couplings through a top quark loop in [24]; we will come back to this below.

Using the contractions in eqs. (4.15), we can now calculate 6-gluon amplitudes up to $\mathcal{O}\left(\alpha^{\prime 5}\right)$ from $(4.9)$. First of all, it is easy to see from that equation that several amplitudes vanish,

$A_{6}^{\mathrm{DBI}}(++++++)=A_{6}^{\mathrm{DBI}}(+++++-)=A_{6}^{\mathrm{DBI}}(-----+)=A_{6}^{\mathrm{DBI}}(------)=0$. 
This follows because there are simply no vertices with six self-dual field strengths or five of them and one anti-selfdual field strength. Therefore, there are no diagrams at tree level. This brings us to the MHV amplitude $A_{6}(++----)$ and its conjugate. There are only two contributions: a direct one $i\left(\pi \alpha^{\prime}\right)^{4} / 2^{8}$ from the vertex in the DBI action (4.9) with six fields, and a contribution which consists of two four-point vertices. In the latter case, two selfdual field strengths need to be contracted. It is easy to see that this yields a contribution exactly proportional to the six-point vertex in the action. In fact, in terms of Wick contractions the relevant part of the calculation is the contraction between the two vertices:

$$
\frac{(i)^{2}}{2} \frac{\pi^{4} \alpha^{\prime 4}}{2^{10}}\left\langle: \text { ext. fields }:: F_{+}^{2} F_{-}^{2}:: F_{+}^{2} F_{-}^{2}:\right\rangle_{0},
$$

where the factor of a $\frac{1}{2}$ comes from the exponential of the action. For a tree level contribution to the amplitude under study, two $F_{+}$have to be contracted between the two vertices. This can be done in 4 different ways. In addition, the 'propagator' from 4.15) gives another factor of $2 i$ after performing the symmetrization. Hence the total prefactor is $-i\left(\pi \alpha^{\prime}\right)^{4} / 2^{8}$ which is the opposite of that of the direct vertex, yielding an exact cancellation off-shell. Therefore, the MHV amplitude is given by

$$
A_{6}^{\mathrm{DBI}}\left(1^{+} 2^{+} 3^{-} 4^{-} 5^{-} 6^{-}\right)=0 .
$$

The conjugate $\overline{\mathrm{MHV}}$ amplitude follows by conjugation and hence also vanishes. This is in line with [28], who argue that only helicity conserving amplitudes are non-zero. Their argument is based on the observation that since the classical equations of motion have a $U(1)$ S-duality symmetry, this symmetry should lead to selection rules. Since the selfdual and anti-selfdual solution have charge +1 and -1 under this symmetry respectively, and are in fact solutions to the full field equations ${ }^{11}$, conservation of the full $U(1)$ implies that only helicity conserving amplitudes are non-zero. This argument is somewhat unusual in that one uses nonperturbative symmetries to prove perturbative results. On the other hand, there are instances in string theory where duality symmetries like S-duality fix parts of the effective action. This in turn fixes the perturbatively derived tree level scattering amplitudes [29]. Regardless of this reasoning, below, we will verify the result explicitly by a diagrammatic argument.

The remaining helicity conserving 6-point NMHV amplitude, $A_{6}^{\mathrm{DBI}}(+++---)$, is slightly more complicated as it will involve one propagator. The result is

$$
A_{6}^{\mathrm{DBI}}\left(1^{+} 2^{+} 3^{+} 4^{-} 5^{-} 6^{-}\right)=-\frac{\pi^{4} \alpha^{\prime 4}}{2^{7}}\left(\frac{[12]^{2}\langle 56\rangle^{2}([4|(1+2)| 3\rangle)^{2}}{\left(p_{1}+p_{2}+p_{4}\right)^{2}}+\text { permutations }\right) \text {. }
$$

Here, the sum is over all permutations that separately permute the positive-helicity indices (123) and the negative helicity-ones (456), but do not mix them. We also use the standard notation

$$
[i|j| k\rangle=\left(p^{i}\right)^{\alpha} p_{\alpha \dot{\alpha}}^{j}\left(p^{k}\right)^{\dot{\alpha}} .
$$

\footnotetext{
${ }^{11}$ Note that (4.8) implies that a variation of the action is proportional to $F^{+} F^{-}$, up to the electromagnetic $F^{2}$ leading term. Hence any self-dual or anti-self-dual field is a solution to the field equations.
} 
Note that this form of the six-point amplitude can be interpreted as just a sum over all the factorization channels: it is easily seen that the residues are the usual four-point functions.

\subsection{Helicity conserving amplitudes from DBI}

After these examples we can proceed to more general cases, but before doing this let us make some simple kinematic observations. The vanishing amplitudes in (4.16) easily generalize to all multiplicity amplitudes, since there are simply no diagrams with the required field strengths on the external lines. This is of course in line with the target space supersymmetric Ward identities which follow from the underlying supersymmetric string theory discussed previously. Furthermore, note that the MHV amplitude is at the very least required to be a 'local' quantity: this quantity cannot have any kinematic poles. Therefore, it must be proportional to the amplitude derived from the vertex with the same number of field strengths as the number of particles in the amplitude, just as was shown above in the case of the six-point amplitude. Actually, the full diagrammatic calculation of an $n$-point

MHV amplitude from the DBI action is structurally the same as that calculation. Hence the MHV amplitude is expected to be

$$
A_{n=\text { even }}^{\text {DBI }}\left(1^{+} 2^{+} 3^{-} \ldots n^{-}\right) \stackrel{?}{\sim}\left(\alpha^{\prime}\right)^{n-2} \sum_{\operatorname{perm}(3, \ldots, n) \notin \text { cyclic }}[12]^{2}\langle 34\rangle^{2}\langle 56\rangle^{2} \ldots\langle n-1, n\rangle^{2},
$$

and only the proportionality constant needs to be calculated. Based on the six-particle result (4.18), one should be starting to suspect that this is actually zero to leading order in $\alpha$ '. For sub-leading orders generated by 'beyond DBI' (higher derivative) contributions to the effective action, the above form may still be relevant.

\section{Vanishing of MHV amplitudes beyond the four-point function (and NMHV beyond six)}

One can show that some of the effects of (4.15) are resummed when one employs a first order formalism. To derive this one first introduces a first order action for the free electromagnetic field pioneered for different purposes by Chalmers and Siegel [30],

$$
S_{\mathrm{CS}}=\int d^{4} x\left[\frac{1}{2} C_{\dot{\alpha} \dot{\beta}} F_{+}^{\dot{\alpha} \dot{\beta}}-\frac{1}{4} C_{\dot{\alpha} \dot{\beta}} C^{\dot{\alpha} \dot{\beta}}\right]
$$

Here $C$ is an auxiliary self-dual two-form. Integrating this out from the above action gives back standard electrodynamics (and a topological term). One can derive the propagators for the above action. In a form similar to (4.15) these read,

$$
\begin{aligned}
& \ldots C_{\dot{\alpha} \dot{\beta}}:: F_{\alpha \beta}^{-} \ldots=i\left(\frac{p_{\alpha \dot{\alpha}} p_{\beta \dot{\beta}}+p_{\beta \dot{\alpha}} p_{\alpha \dot{\beta}}}{p^{2}}\right) \\
& \ldots C_{\dot{\alpha} \dot{\beta}}:: C_{\dot{\delta} \dot{\gamma}} \ldots=0 \\
& \ldots F_{\alpha \beta}^{-}:: F_{\delta \gamma}^{-} \ldots=-i\left(\epsilon_{\alpha \delta} \epsilon_{\beta \gamma}+\epsilon_{\alpha \gamma} \epsilon_{\beta \delta}\right)
\end{aligned}
$$

The second line above is the most important one: in this first order form contractions between $C$ fields (which are $F_{+}$on-shell) are trivial. Inspired by (4.22), it is easy to see 
that in order to obtain (4.10) one needs to integrate out $C$ from

$$
S=\int d^{4} x\left[\frac{1}{2} C_{\dot{\alpha} \dot{\beta}} F_{+}^{\dot{\alpha} \dot{\beta}}-\frac{1}{4}\left(1+\alpha^{\prime 2} \pi^{2} \frac{1}{8} F_{-}^{2}\right) C_{\dot{\alpha} \dot{\beta}} C^{\dot{\alpha} \dot{\beta}}+\mathcal{O}\left(F_{+}^{4}\right)\right] .
$$

This form makes it obvious that the 4-point MHV amplitude is the only MHV amplitude: the perturbation series works analogous to the case considered above, except for the second line in (4.23) and the absence of vertices with one $F_{+}^{2}$ and multiple $F_{-}^{2}$ in the action. It is easy to see that this action only leads to 4-point MHV and 6-point NMHV amplitudes. More precisely,

$$
A^{\mathrm{DBI}}\left(++-^{i}\right)=A^{\mathrm{DBI}}\left(+++-^{i+1}\right)=0 \quad \forall i>2 .
$$

In principle this argument can be extended to NNMHV and beyond, but below we follow a more elegant and parity-symmetric route.

This form of the action is notable for being easily lifted to twistor space. Just as the Poincaré group acts linearly on $\mathbb{R}^{4}$, the complexification of the conformal group of this four-dimensional space acts linearly on $\mathbb{C P}^{3}$, which is known as its twistor space. Given an action on space-time with spin $0, \frac{1}{2}, 1$ fields, it is possible to construct an action on twistor space which reproduces the perturbation series of the original action, see for instance the review in [24]. The resulting twistor action generates exactly one MHV amplitude: the four-point one, while the rest vanishes. Unfortunately, the higher-order terms seem to defy a neat formulation on the twistor space. Also, the parity-symmetric structure of the amplitudes uncovered below suggests that one should be looking for ambi-twistor space action constructions [31] which are not nearly as well understood.

\section{Helicity conservation}

In the following we will find it useful to consider not the DBI action in the form (4.2), but written using two auxiliary complex scalar fields [32]:

$$
\mathcal{L}_{\mathrm{DBI}}=-\frac{1}{4} F_{+}^{2}+\frac{i}{2 \pi \alpha^{\prime}}\left(-i a \bar{a}+\lambda a-\bar{\lambda} \bar{a}+\frac{\sqrt{\pi \alpha^{\prime}}}{2} a \bar{a}(\lambda-\bar{\lambda})\right)-i \frac{\sqrt{\pi \alpha^{\prime}} \lambda}{8} F_{+}^{2}+i \frac{\sqrt{\pi \alpha^{\prime}} \bar{\lambda}}{8} F_{-}^{2},
$$

Shifting the field $\lambda$ by $-i / \sqrt{\pi \alpha^{\prime}}$ and integrating out the $\lambda$ and $a$ fields yields back (4.2). The main reason this is an easier action to consider is that the coupling of the field strengths is only through scalars. In fact, this exact same coupling is considered in the literature for non-Abelian fields for effective Higgs-gluon couplings through a top quark loop [33]. It is not hard to see that since the contractions between like-selfduality field strengths happen off-shell, one can resum these effects into new effective vertices. It is then important to calculate the correct constants multiplying these effective vertices. These can be read of from 24] by specializing to an Abelian gauge group ${ }^{12}$. More specifically, in our conventions one can replace

$$
-i \frac{\sqrt{\pi \alpha^{\prime}} \lambda}{8} F_{+}^{2} \rightarrow-\frac{1}{4} \frac{i \sqrt{\pi \alpha^{\prime}} \lambda}{2+i \sqrt{\pi \alpha^{\prime}} \lambda}: F_{+}^{2}: \quad i \frac{\sqrt{\pi \alpha^{\prime}} \bar{\lambda}}{8} F_{-}^{2} \rightarrow \frac{1}{4} \frac{i \sqrt{\pi \alpha^{\prime}} \bar{\lambda}}{2-i \sqrt{\pi \alpha^{\prime}} \bar{\lambda}}: F_{-}^{2}:,
$$

\footnotetext{
${ }^{12}$ Using the correct normalization of the color matrices there.
} 


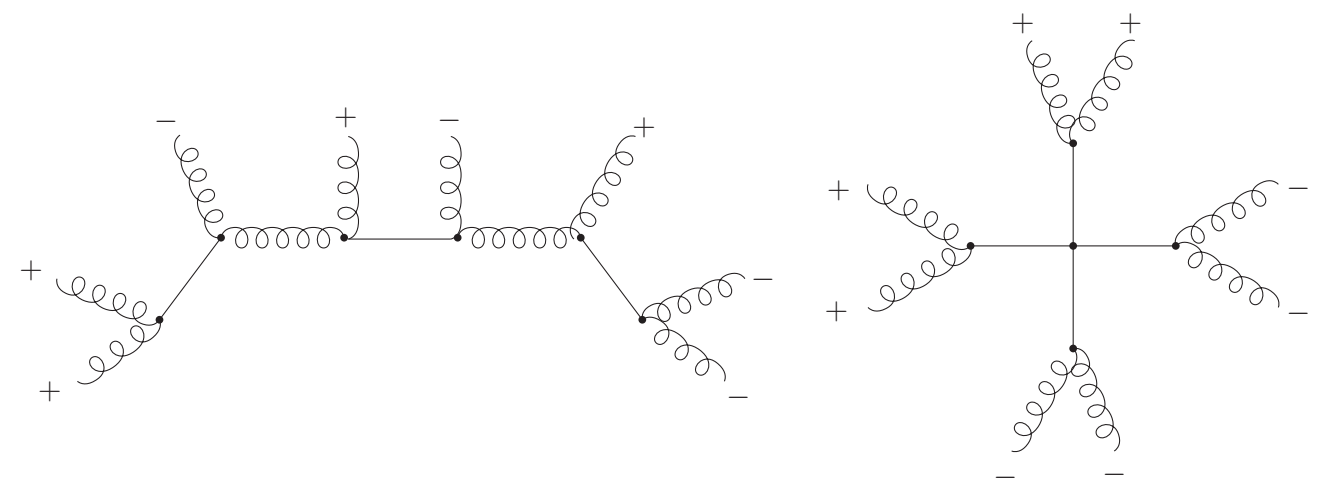

Figure 2: Illustration of the two types of contribution to the 8-point amplitude: non-local (left) and local (right).

as long as one keeps in mind that contractions between like-selfduality field strengths are not allowed any more; only the non-local contraction between $F_{-}$and $F_{+}$is still allowed.

Now we turn to the scalar part of (4.26). The fields $a$ and $\bar{a}$ can be integrated out exactly, which yields the following polynomial scalar Lagrangian

$$
\mathcal{L}_{\text {scalar }}=-\frac{1}{\pi \alpha^{\prime}}\left(\frac{\lambda \bar{\lambda}}{2+\sqrt{\pi \alpha^{\prime}} i(\lambda-\bar{\lambda})}\right)
$$

Given the couplings in (4.27), it is natural to define new fields $k$ and $\bar{k}$ as

$$
k=\frac{\lambda}{2+i \sqrt{\pi \alpha^{\prime}} \lambda} \quad \bar{k}=\frac{\bar{\lambda}}{2-i \sqrt{\pi \alpha^{\prime}} \bar{\lambda}} .
$$

Plugging this field redefinition into the scalar part (4.28), one obtains

$$
\mathcal{L}_{\mathrm{DBI}}=-\frac{1}{\pi \alpha^{\prime}}\left(\frac{2 k \bar{k}}{1-\pi \alpha^{\prime} k \bar{k}}\right)-\frac{1}{8} i \sqrt{\pi \alpha^{\prime}} k: F_{+}^{2}:+\frac{1}{8} i \sqrt{\pi \alpha^{\prime}} \bar{k}: F_{-}^{2}:
$$

This form of the DBI action is very convenient for deriving tree level helicity amplitudes. Note that its Feynman rules are such that only equal numbers of selfdual and anti-selfdual field strengths appear on the external legs. This is a direct diagrammatic verification of the claim (see also section 4.2) in [28]: amplitudes derived from the DBI action conserve helicity.

Loop level amplitudes are ill-defined for this action in general, not taking into account the fact that in general loop calculations in an effective action only estimate the effects of the UV theory. However, knowing that amplitudes vanish at tree level implies that several loop level amplitudes are rational functions of the external momenta as they cannot have branch cuts. This seems to imply some of the annulus diagrams might also be simpler than expected. Note that most of the Jacobians encountered in the field transformations above will vanish in dimensional regularization as they involve $\int d^{d} p 1=0$. 


\subsection{Interpretation as (partial) CSW rules}

The non-zero amplitudes also follow from this action. As a cross-check, the six-point amplitude (4.19) calculated above is seen to be reproduced (the form of the diagrams is illustrated for different purposes in figure 5, see appendix B). For the eight-point amplitude there are only two types of contribution illustrated in figure 2. The type displayed on the left in that figure is the generalization of (4.19), which can again be interpreted as a sum over factorization channels. The other is a direct contribution from the action generated by the helicity conserving four-point scalar vertex in 4.30)

$$
A_{8}^{\mathrm{DBI}}(++++----)_{\text {local }} \sim \pi^{6}{\alpha^{\prime}}^{6} \sum_{\text {perm(1234)(5678) }}\langle 12\rangle^{2}\langle 34\rangle^{2}[56]^{2}[78]^{2} .
$$

This also illustrates to what extent the DBI action generates 'CSW'-style perturbation theory rules. For our purposes, this can be phrased as the question if given all $\mathrm{N}^{i} \mathrm{MHV}$ amplitudes (not vertices) one can calculate the $\mathrm{N}^{i+1} \mathrm{MHV}$ amplitudes. This question is however rather simple considering the perturbation theory derived from (4.30) (which is already quite close) since there are not that many non-zero contributions.

Given the MHV amplitude the NMHV amplitude can be calculated. For this one uses the MHV amplitudes as vertices and recognizes that the spinor products of the leg which needs to be taken off-shell exactly correspond to the product of the Lorentz momentum flowing through the leg. One replaces the Lorentz momentum by the off-shell Lorentz momentum and arrives at the same expression as generated by the action (4.30). Unfortunately this process does not generate the complete NNMHV amplitude because of the 'local' contribution calculated in (4.31). The extra contributions are known though, since for

$$
n=4 m \quad, m>1 \text {, }
$$

fields all these can be calculated explicitly from combining four scalar vertices leading to the natural generalization of (4.31),

$$
A_{2 i}^{\mathrm{DBI}}\left((+)^{i}(-)^{i}\right)_{\mathrm{local}} \sim\left(\pi \alpha^{\prime}\right)^{2(i-1)} \sum_{\operatorname{perm}(1 \ldots i)(i+1 \ldots 2 i)}\langle 12\rangle^{2} \ldots\langle i-1, i\rangle^{2}[i+1, i+2]^{2} \ldots[2 i-1,2 i]^{2},
$$

where $i=2 m$. For example for twelve particles these come from diagrams with two fourpoint vertices, etc. On the other hand for

$$
n=4 m+2 \quad, m \geq 1
$$

the Dirac-Born-Infeld action does obey CSW-style Feynman rules directly. The more important observation though is that since so many amplitudes vanish, the perturbation theory is surprisingly trivial.

\section{More general amplitudes}

Applying the usual supersymmetric Ward identities to the derived scattering amplitude formulae in this section will generate certain amplitudes with specific combinations of 
quarks and scalars. These amplitudes are generated from the supersymmetric completion of the DBI action as written above. Note that since the supersymmetric partners of the photon transform in the adjoint of the gauge group, one obtains uncharged scalars and fermions and one therefore expects no extra contributions at order $\alpha^{\prime 0}$ from these particular terms. There are more terms however, which arise for scalars for instance directly under the 'square root' sign by dimensional reduction from the ten-dimensional DBI action. It would be interesting to study their coupling further.

\section{Higher orders in $\alpha^{\prime}$}

As noted in the introduction to this section, several string corrections to the DBI action are known. These are basically the series of term related to the Veneziano amplitude which have four fields and those terms in the effective action which have 4 derivatives. Although both of these are interesting we will only make some preliminary remarks about both of them.

\section{Further remarks}

In principle, since the expression in (4.26) is highly reminiscent of open string field theory one might suspect that one can calculate also the above mentioned corrections with an action of the same type. The starting point is writing a kinetic term for the scalars, after which counter terms must be added to obtain the correct correction to the Veneziano amplitude (which starts at order $\alpha^{4}$ ). These terms may involve massive spin fields with spin to mimic the states of the string. The problem is that although constructing an extension of (4.26) which reproduces the Veneziano amplitude along these lines is possible in principle, in practice this procedure is fairly arbitrary. Note that related observations play a major role in appendix B, which discusses on-shell recursion for the DBI action.

More promising, one can construct a similar action as in (4.26) which reproduces the non-Abelian DBI action up to $\alpha^{\prime 2}$. In this respect it is interesting to note that although there is no gauge invariant derivative expansion, there is a gauge invariant expansion in terms of angular momentum of the modes of the massive states.

\section{BCFW recursion relations in string theory}

In this section we initiate the study of on-shell recursion relations of the BCFW type for string theory amplitudes. First we review the general derivation of on-shell recursion relations, followed by some general comments. After this we study some examples for which everything can be checked explicitly. Note that this section would do well in any seventiesstring-theory-buzzword-bingo game as it involves concepts like duality, Regge behavior and resonance poles.

Before continuing let us note that at the very least the $\alpha^{\prime 2}$ correction obeys some recursion relation: by it's close relation to the one loop all-plus gluon amplitude in pure Yang-Mills theory, it will obey the same recursion relations as studied in [34]. However, these were derived 'experimentally' by studying the residue at infinity for a given BCFWlike shift of the known expression. It would be interesting to see if a similar procedure 
would work for our $\alpha^{\prime 3}$ result in section 3. For the rest of this section, we focus on BCFW's original idea.

\subsection{The main BCFW idea}

Following BCFW's idea [4] quite closely, the momenta of 2 external particles which can be massive or massless in $D \geq 4$ dimensions are shifted by a vector $n_{\mu}$

$$
\begin{aligned}
p_{i}^{\mu} \rightarrow \hat{p}_{i}^{\mu} & =p_{i}^{\mu}+z n^{\mu} \\
p_{j}^{\mu} \rightarrow \hat{p}_{j}^{\mu} & =p_{j}^{\mu}-z n^{\mu},
\end{aligned}
$$

which preserves momentum conservation. Furthermore, $n^{\mu}$ must be such that these momenta stay on-shell. Therefore the vector $n$ must obey

$$
\left(p_{i}^{\mu} n_{\mu}\right)=\left(p_{j}^{\mu} n_{\mu}\right)=\left(n^{\mu} n_{\mu}\right)=0 .
$$

These equations do not have a solution for real $n_{\mu}$, but do for complex momenta, as can be easily verified by going to the center-of-mass frame. This shift changes the amplitude into a function of $z$.

The amplitude we are interested in is given by $A_{n}(z=0)$, which can be obtained by a contour integration around a contour which only encompasses $z=0$,

$$
A_{n}(0)=\oint_{z=0} \frac{A_{n}(z)}{z} d z
$$

If the contour is now pulled to infinity one encounters various poles at finite values of $z$ and a possible residue at infinity,

$$
A_{n}(0)=\oint_{z=0} \frac{A_{n}(z)}{z} d z=-\left\{\sum \operatorname{Res}_{z=\text { finite }}+\sum \operatorname{Res}_{z=\infty}\right\} .
$$

In a unitary theory, the only poles possible at finite $z$ are those at which physical particles become on-shell. Tree level unitarity then guarantees that the residue at such a pole is the product of two scattering amplitudes. In string theory at the disk level this is (almost) manifest by the worldsheet 'pinching' argument illustrated in figure 1. Note that the residues at the finite values of $z$ will involve scattering amplitudes evaluated for shifted values of the momenta. Therefore, provided one can ignore the residue at infinity, one arrives at recursion relations of the form

$$
A_{n}(1,2,3 \ldots, n)=\sum_{r, h(r)} \sum_{k=2}^{n-2} \frac{A_{k+1}\left(1,2, \ldots, \hat{i}, \ldots, k, \hat{P}_{r}\right) A_{n-k+1}\left(\hat{P}_{r}, k+1, \ldots, \hat{j}, \ldots, n\right)}{\left(p_{1}+p_{2}+\ldots+p_{k}\right)^{2}+m_{r}^{2}} .
$$

Here the label $r$ runs over all the states in the string theory, $m_{r}$ is the corresponding mass and $h(r)$ symbolizes the sum over all possible polarizations of the state. The momentum $\hat{P}_{r}$ for the 'extra' particle and its anti-particle in the amplitude is such that the particle is on-shell. Note that in this expression one does not sum over all kinematic channels: only those momentum invariants which become $z$-dependent under the shift (5.1) can generate 
poles. Hence one does not obtain poles in the channels for which the momentum invariant involves the sum of the shifted momenta, since

$$
\hat{p}_{i}^{\mu}+\hat{p}_{j}^{\mu}=p_{i}^{\mu}+p_{j}^{\mu}
$$

Therefore, while the resulting expression (5.5) incorporates some kinematic poles explicitly, it seems to lack others. As will be shown below, this remark ties in very closely with the old concept of 'duality' in string theory.

In the following we will focus on proving in specific examples that the residue at infinity is absent, thereby proving the recursion relations. In passing it is noted that in the literature one often refers to a 'pole' at infinity spoiling recursion relations. However, since there is a possibility that a function has a pole at infinity but no residue, it is better to refer to this as a 'residue at infinity'.

\section{General comments about BCFW recursion in string theory}

Since any (non-topological) string theory has an infinite tower of massive states, there will in general be various infinite summations throughout the calculation for poles at finite $z$ corresponding to both the infinite set of massive states and the sums over the polarization tensors of these massive states. This is a serious drawback limiting the current calculational usefulness of the method and we will make some comments about this further along in this section. Another potential drawback of these recursion relations is that when one starts with for instance a purely gluonic amplitude, then after applying the recursion once one will need generically 2 amplitudes which involve one massive state each and a sum over its polarization tensors. Therefore, the set of gluon amplitudes is generically not closed under recursion, although the ordinary BCFW relations form a subset of them and will be obtained in an $\alpha^{\prime} \rightarrow 0$ limit. A comment related to this limit is that all the massive poles will be at certain integer multiples of the string mass. Generically, this will look like

$$
\left(\mathcal{P}+\hat{p}_{i}\right)^{2}=\frac{k}{\alpha^{\prime}}
$$

where $\mathcal{P}$ is a sum over momenta which does not involve $\hat{p}_{j}$ and $k$ is a negative integer. The corresponding value of $z$ is

$$
z_{\text {pole }}=\frac{1}{2(n \cdot \mathcal{P})}\left(\frac{k}{\alpha^{\prime}}-\left(\mathcal{P}+p_{i}\right)^{2}\right) .
$$

Hence, these poles will move to $z=\infty$ if $\alpha^{\prime} \rightarrow 0$, leading generically to non-zero residues there. This is also to be expected: as pointed out in [35], one can interpret the shift (5.1) for very large values of $z$ as describing a hard particle moving through a soft background. The $\alpha^{\prime} \rightarrow 0$ limit should more appropriately be described without dimensions as the limit in which all momentum invariants are smaller than 1 . Hence first taking this limit and then studying a 'hard' particle for which some momentum invariants will be larger than one will generically lead to inconsistencies: these are precisely contained in the poles at infinity. Hence one should always first derive recursion relations and then apply an expansion in $\alpha^{\prime}$, if possible. This is related to the fact that the $z \rightarrow \infty$ limit is thought to be connected to 
the UV properties of the theory under study: while string theory has excellent behavior in the UV being (at least one (string)loop) finite, any effective field theory derived from the string will generically have bad behavior since it is non-renormalisable. Note that this is also a hand-waving argument why the residue at infinity should be expected to be absent for any string theory amplitude; that is, for any amplitude for any particle type in any unitary string theory.

Another indirect argument for the correctness of (5.5) is that repeated application of this relation (i.e. disregarding any potential residues at infinity) allows one to express any string theory amplitude in terms of just three-point on-shell amplitudes. This is reminiscent of open bosonic string field theory, although making this precise would probably require much work. On the other hand, note that closed bosonic string field theory does not share this property. We will take both the open string field theory argument and more importantly the UV behavior argument as a prime indication that string theory amplitudes may obey (5.5). As emphasized, the verification of this statement depends on the absence of residues at infinity: we will verify this explicitly in a number of examples.

\subsection{Veneziano amplitude}

Before proceeding to a more general argument and superstring gluon amplitudes, it is instructive to note that even the mother of all string theory amplitudes obeys recursion relations. This is of course the Veneziano amplitude for four tachyons in open bosonic string theory. The full Veneziano amplitude is simply given by

$$
A_{4}=A_{4}^{\mathrm{part}}(s, t)+A_{4}^{\mathrm{part}}(t, u)+A_{4}^{\mathrm{part}}(u, s),
$$

where

$$
A_{4}^{\mathrm{part}}(s, t)=\frac{\Gamma\left(\alpha^{\prime} s-1\right) \Gamma\left(\alpha^{\prime} t-1\right)}{\Gamma\left(\alpha^{\prime}(s+t)-2\right)} .
$$

Here $s=\left(p_{1}+p_{2}\right)^{2}, t=\left(p_{1}+p_{3}\right)^{2}$ and $u=\left(p_{2}+p_{3}\right)^{2}$ are the customary Mandelstam variables. This formula and its $n$-particle generalizations have several remarkable properties detailed in the literature, see e.g. [36] for a recent review. Here a shift of particles 1 and 2 is considered for which

$$
\hat{s}=s, \quad \hat{t}=t-2 z\left(p_{3}^{\mu} n_{\mu}\right), \quad \hat{u}=u+2 z\left(p_{3}^{\mu} n_{\mu}\right) .
$$

By construction, the vector $n_{\mu}$ obeys equation (5.2). Note that every other shift is equivalent by momentum conservation. Before continuing it is convenient to lose the $2\left(p_{3}^{\mu} n_{\mu}\right)$ factor altogether by rescaling the integration variable $z$,

$$
z^{\prime}=2 \alpha^{\prime}\left(p_{3}^{\mu} n_{\mu}\right) z
$$

Note that the fact that this can be done is a kinematical accident for four particles.

There are two different types of term in (5.9) after the shift (5.11): there is one term in which two momentum invariants $(t, u)$ are shifted, and two terms in which one momentum 
invariant is shifted. Let us first consider the last type of contribution, say the first term in (5.9) for which (5.4) gives

$$
A_{4}^{\text {part }}(s, t)=-\operatorname{Res}_{z^{\prime}=\infty}\left(\frac{A_{4}^{\text {part }}\left(s, t, z^{\prime}\right)}{z^{\prime}}\right)+\sum_{n=0}^{\infty} \frac{(-1)^{n}}{\Gamma(n+1)} \frac{\Gamma\left(\alpha^{\prime} s-1\right)}{\Gamma\left(\alpha^{\prime} s-1-n\right)} \frac{1}{\alpha^{\prime} t-1+n} .
$$

As long as $\alpha^{\prime} s$ is not evaluated at one of the resonances, the infinite sum on the right hand side of this equation is equivalent to $A_{4}^{\text {part }}(s, t)$ by an identity for the Beta function. This identity can for instance be derived by using the integral representation $B(a, b)=$ $\int_{0}^{1} x^{a-1}(1-x)^{b-1}$ and expanding $(1-x)^{b-1}$ using Newton's binomial theorem. Note that this particular identity appears for instance in [13], eq. (1.1.15), which identifies that equation as BCFW on-shell recursion avant la lettre. This proves explicitly in this particular case that

$$
\operatorname{Res}_{z^{\prime}=\infty}\left(\frac{A_{4}^{\mathrm{part}}\left(s, t, z^{\prime}\right)}{z^{\prime}}\right)=0 .
$$

The same argument goes through for the third term in (5.9), $A_{4}^{\text {part }}(u, s)$. Actually, the infinite summation in the sum in (5.13) is usually used to illustrate the 'duality' of an open string amplitude: the infinite series has only explicit poles in $t$, but when summed also exhibits poles in $s$. This is just the observation that a string worldsheet can be pinched in multiple ways.

The contribution for which two momentum invariants are shifted, $A_{4}^{\text {part }}(u, t)$, is more tricky as it reads,

$$
\begin{aligned}
A_{4}^{\mathrm{part}}(u, t)= & -\operatorname{Res}_{z^{\prime}=\infty}\left(\frac{A_{4}^{\mathrm{part}}\left(u, t, z^{\prime}\right)}{z^{\prime}}\right) \\
& +\sum_{n=0}^{\infty} \frac{(-1)^{n}}{\Gamma(n+1)} \frac{\Gamma\left(\alpha^{\prime}(u+t)-2+n\right)}{\Gamma\left(\alpha^{\prime}(u+t)-2\right)}\left(\frac{1}{\alpha^{\prime} t-1+n}+\frac{1}{\alpha^{\prime} u-1+n}\right) .
\end{aligned}
$$

This particular sum equals $A_{4}^{\text {part }}(u, t)$ again as can be checked directly through a calculation in Maple. Hence

$$
\operatorname{Res}_{z^{\prime}=\infty}\left(\frac{A_{4}\left(u, t, z^{\prime}\right)}{z^{\prime}}\right)=0 .
$$

Note that even without Maple, one can check that does have all the right poles. In addition, we have checked analytically up to order $\alpha^{1}$ and numerically up to order $\alpha^{10}$ that this is true in the sense that numerically the difference between the sum and the left hand side seems to converge to zero by taking into account more and more terms of the sum, leaving $u, t$ as free variables. Furthermore, in the case $u=-t$ it can be proven by hand since then on the left hand side of the equation Euler's reflection identity,

$$
\Gamma(z) \Gamma(-z)=-\frac{\pi}{z \sin (\pi z)},
$$

can be used, while the right hand side can be explicitly molded into the Laurent series of this function. Note that a general order by order in $\alpha^{\prime}$ comparison involves very interesting identities between sums over (powers of) harmonic numbers and explicit zeta function 
values, also known as Euler sums. We are actually unsure whether all of these are known. Note that in a color ordered amplitude all three contributions in (5.9) would be independent, so it is encouraging that the residue at infinity terms vanishes separately for these three terms. However, one would like a more direct argument for the absence of residues at infinity so the above result could be derived. This will be constructed below.

\subsection{Absence of residues at infinity for four-point functions}

For the above shifts in the $(s, t)$ channel one can study the integral at infinity directly ${ }^{13}$. For this integral to be well-defined, let us pull the contour in (5.4) to a large but finite value of $z^{\prime}$. For definiteness, let us specify the contour as a circle of radius $R_{k}$, where $R_{k}$ is a half-integer $\left(R_{k}=k+\frac{1}{2}, k \in \mathbb{N}\right)$, so we obtain

$A^{\mathrm{part}}(0)(s, t)=\sum_{n=0}^{k} \frac{(-1)^{n}}{\Gamma(n+1)} \frac{\Gamma\left(\alpha^{\prime} s-1\right)}{\Gamma\left(\alpha^{\prime} s-1-n\right)} \frac{1}{\alpha^{\prime} t-1+n}+\oint_{C_{R_{k}}} \frac{1}{z^{\prime}} \frac{\Gamma\left(\alpha^{\prime} s-1\right) \Gamma\left(\alpha^{\prime} t+z^{\prime}-1\right)}{\Gamma\left(\alpha^{\prime}(s+t)+z^{\prime}-2\right)} d z$.

For very large radius, we can approximate the integrand of the contour integral by a wellknown formula

$$
\frac{1}{z^{\prime}} \frac{\Gamma\left(\alpha^{\prime} t+z^{\prime}-1\right)}{\Gamma\left(\alpha^{\prime}(s+t)+z^{\prime}-2\right)}=\left(z^{\prime}\right)^{-\alpha^{\prime} s}\left(1+\frac{1}{2 z^{\prime}}\left(1-\alpha^{\prime} s\right)\left(2 \alpha^{\prime} t+\alpha^{\prime} s-3\right)+\mathcal{O}\left(\frac{1}{\left(z^{\prime}\right)^{2}}\right)\right) .
$$

This formula is usually used to display Regge behavior of the string theory amplitudes. It is actually only valid for $-\pi<\arg (z)<\pi$, but the missing point may simply be deleted from the contour integral as it is a measure zero set. Since the integrand is certainly analytic in a neighborhood of this point, there is no possibility of a contribution to the integral from this set. The resulting elementary integral can be calculated since generally,

$$
\oint_{C_{R}} \frac{1}{z^{a}} d z=i R^{1-a} \int d \theta e^{i(1-a) \theta}=R^{1-a} \frac{1}{1-a}\left(e^{i 2 \pi(1-a)}-1\right) .
$$

Hence, as long as Re $(1-a)<0$ the elementary integral vanishes for large radius. In the case under consideration, this translates to

$$
\operatorname{Re}\left(\alpha^{\prime} s\right)>1 .
$$

Note that this condition neatly evades all the resonant poles in the amplitude. This is also needed as the sum does not contain explicit poles in the $s$ channel.

For the shift in the $(u, t)$ contribution one obtains similarly the integral

$$
\operatorname{Res}_{z^{\prime}=\infty}\left(\frac{A_{4}^{\mathrm{part}}\left(u, t, z^{\prime}\right)}{z^{\prime}}\right)=\oint_{C_{R}} \frac{1}{z^{\prime}} \frac{\Gamma\left(\alpha^{\prime} u-z^{\prime}-1\right) \Gamma\left(\alpha^{\prime} t+z^{\prime}-1\right)}{\Gamma\left(\alpha^{\prime}(u+t)-2\right)} d z .
$$

Using (5.17), this can be written as

$$
\begin{aligned}
\operatorname{Res}_{z^{\prime}=\infty}\left(\frac{A_{4}^{\mathrm{part}}\left(u, t, z^{\prime}\right)}{z^{\prime}}\right)=\oint_{C_{R}} \frac{1}{z^{\prime}} & \frac{1}{\Gamma\left(\alpha^{\prime}(u+t)-2\right)} \frac{\Gamma\left(\alpha^{\prime} t+z^{\prime}-1\right)}{\Gamma\left(1-\alpha^{\prime} u+z^{\prime}\right)} \\
& \frac{\pi}{\left(\alpha^{\prime} u-z^{\prime}-1\right) \sin \left(\pi\left(\alpha^{\prime} u-z^{\prime}-1\right)\right)} d z .
\end{aligned}
$$

\footnotetext{
${ }^{13}$ taking inspiration from [37], section 1.4.
} 
For large $z^{\prime}$, we can apply (5.19) again. However, the secant function also has a non-trivial large $z^{\prime}$ expansion as it vanishes exponentially along any direction not along the real axis, and is analytic along that axis. Hence the behavior at infinity is actually far better than for the other case: the integrand vanishes exponentially on the contour up to a measure zero set (supported on two points in this case), in the neighborhood of which the integrand is analytic. This reflects itself in the fact that in this case the resulting recursion relation, (5.15), contains the two possible series of poles explicitly so there is no need for a restriction like (5.21). Hence (5.16) is actually true without any provisos.

These results have a very important immediate extension: if the Beta function had been multiplied by any rational function of $z^{\prime}$ or more generally, any function which has a Laurent series at infinity, the same result would follow. The only thing which changes is that if the prefactor diverges, $\sim z^{k}$ for some positive $k$, then the constraint (5.21) on $\alpha^{\prime} s$ would change slightly. However, as long as the appropriate reality condition is satisfied, there are no problems. Hence we expect that the above derivation holds for any four particle amplitude in any open string theory with arbitrary external states. In particular, we expect recursion to work for the gluon amplitude in the superstring, as we will verify below. Some subtleties related to the $\alpha^{\prime}$ expansion will also be discussed there.

\section{Closed strings: Virasoro-Shapiro amplitude}

Although this article mainly deals with open strings, it is hard to resist generalizing the above derivation of the absence of a residue at infinity to the scattering of four closed string tachyons in bosonic string theory. This particular amplitude is known as the VirasoroShapiro amplitude and is given by,

$$
A_{4}(s, t, u)=\frac{\Gamma\left(\frac{\alpha^{\prime} s-1}{2}\right) \Gamma\left(\frac{\alpha^{\prime} t-1}{2}\right) \Gamma\left(\frac{\alpha^{\prime} u-1}{2}\right)}{\Gamma\left(1-\frac{\left(\alpha^{\prime} s-1\right)}{2}\right) \Gamma\left(1-\frac{\left(\alpha^{\prime} t-1\right)}{2}\right) \Gamma\left(1-\frac{\left(\alpha^{\prime} u-1\right)}{2}\right)} .
$$

The previous analysis of the shift in the $(u, t)$ channel for the open string amplitude, resulting in (5.15), can be adapted for the above amplitude using the same shift. One then finds

$$
\begin{aligned}
& A_{4}(s, t, u)=-\operatorname{Res}_{z^{\prime}}=\infty\left(\frac{A_{4}\left(s, t, u, z^{\prime}\right)}{z^{\prime}}\right) \\
& \quad+\sum_{n=0}^{\infty} \frac{(-1)^{n}}{\Gamma(n+1)} \frac{\Gamma\left(\frac{\alpha^{\prime} s-1}{2}\right)}{\Gamma\left(1-\frac{\left(\alpha^{\prime} s-1\right)}{2}\right)} \frac{\Gamma\left(\frac{\alpha^{\prime}(t+u)-2+n}{2}\right)}{\Gamma\left(2-\frac{\left[\alpha^{\prime}(t+u)+n\right]}{2}\right)}\left(\frac{1}{\alpha^{\prime} t-1+n}+\frac{1}{\alpha^{\prime} u-1+n}\right) .
\end{aligned}
$$

We have not been able to analytically evaluate the sum in this expression, but we have checked numerically that the difference between the sum and the left hand side seems to converge to zero by taking into account more and more terms of the sum. One can also show the absence of the residue at infinity more directly, following the same argument as 
above by considering

$$
\begin{aligned}
\operatorname{Res}_{z^{\prime}=\infty}\left(\frac{A_{4}\left(s, t, u, z^{\prime}\right)}{z^{\prime}}\right) & =\frac{\Gamma\left(\frac{\alpha^{\prime} s-1}{2}\right)}{\Gamma\left(\frac{3-\alpha^{\prime} s}{2}\right)} \operatorname{Res}_{z^{\prime}=\infty}\left(\frac{1}{z^{\prime}} \frac{\Gamma\left(\frac{\alpha^{\prime} t-1}{2}\right) \Gamma\left(\frac{\alpha^{\prime} u-1}{2}\right)}{\Gamma\left(\frac{3-\alpha^{\prime} t-z^{\prime}}{2}\right) \Gamma\left(\frac{3-\alpha^{\prime} u+z^{\prime}}{2}\right)}\right) \\
& =\frac{\Gamma\left(\alpha^{\prime} s-1\right)}{\Gamma\left(\frac{3-\alpha^{\prime} s}{2}\right)} \operatorname{Res}_{z^{\prime}=\infty}\left(\frac{1}{z^{\prime}}\left(\frac{z^{\prime}}{2}\right)^{-\frac{\alpha^{\prime} s}{2}}\left(-\frac{z^{\prime}}{2}\right)^{-\frac{\alpha^{\prime} s}{2}}\left(1+\mathcal{O}\left(\frac{1}{z^{\prime}}\right)\right)\right) .
\end{aligned}
$$

For an appropriate reality condition on $s$, the integrand vanishes at infinity as above. In addition, the integrand is a well-defined analytic function in the neighborhood of the measure zero set on which the asymptotic series is not valid. Hence the residue integral at infinity vanishes for this particular amplitude and the Virasoro-Shapiro amplitude obeys on-shell recursion relations. Although we will not write them out explicitly here, note that the infinite sum one obtains indeed incorporates all the poles of the full amplitude explicitly. By the same extension as before, any rational function of $z^{\prime}$ times (5.24) is therefore also expected to obey recursion relations since the residue at infinity is absent there. This argument therefore applies to all closed string four-point amplitudes, as they have this generic shape.

\section{Extension to higher point functions}

Higher point functions are more complicated, but the general argument given above should continue to hold. The reason this is the case is that the generic integral appearing for, say the $n$-point function can always be decomposed into infinite sums over Gamma functions. Assuming the argument given above continues to commute with the infinite sums, it is very hard to see what could go wrong. Of course, it would be very interesting to make this more precise, especially as we will see that the recursion relation even for the 5-gluon amplitude is fairly non-trivial.

\subsection{The four- and five-point gluon amplitudes}

As detailed earlier in equation (2.3), the 4-point gluon amplitude in four dimensions is given by

$$
A_{4}\left(1^{-}, 2^{-}, 3^{+}, 4^{+}\right)=\frac{\langle 12\rangle^{4}}{\langle 12\rangle\langle 23\rangle\langle 34\rangle\langle 41\rangle} \frac{\Gamma\left(1+\alpha^{\prime} s\right) \Gamma\left(1+\alpha^{\prime} t\right)}{\Gamma\left(1+\alpha^{\prime} s+\alpha^{\prime} t\right)} .
$$

We can now follow the same steps as above using again the shifts in eq. (5.11).

\section{A subtlety}

At this point one should be slightly more careful about the helicities of the states in the gluon amplitude when applying the shift. In four dimensions, there are two solutions to the constraints (5.2) for the vector $n^{\mu}$ :

$$
n^{\mu}=p_{1}^{\alpha} p_{2}^{\dot{\alpha}} \quad \text { or } \quad n^{\mu}=p_{2}^{\alpha} p_{1}^{\dot{\alpha}}
$$


We will pick the first one, so that the spinors transform as

$$
\begin{aligned}
& p_{1}^{\dot{\alpha}} \rightarrow p_{1}^{\dot{\alpha}}-z p_{2}^{\dot{\alpha}} \\
& p_{2}^{\alpha} \rightarrow p_{2}^{\alpha}+z p_{1}^{\alpha} .
\end{aligned}
$$

This leads to the transformations

$$
s \longrightarrow s, \quad t \longrightarrow t-z\langle 13\rangle[23] \text { and }\langle 23\rangle \longrightarrow\langle 23\rangle+z\langle 13\rangle .
$$

Now one finds by explicit inspection of the Yang-Mills factor in (5.27) that it vanishes for $z \rightarrow \infty$ if and only if the helicities of 1 and 2 are $(+,+),(-,-)$ or $(+,-)$ respectively. The shift in helicities $(-,+)$ is 'well-behaved' for the other solution in (5.28). However, as we will see below, in string theory the situation is better: also the bad shift actually works.

\section{Recursion}

Below we focus on the $A_{4}(--++)$ amplitude; the other cases are similar, keeping the above subtlety in mind. Applying the transformations (5.30) to (5.27) one finds

$$
A_{4}(z)=\frac{\langle 12\rangle^{4}}{\langle 12\rangle(\langle 23\rangle+z\langle 13\rangle)\langle 34\rangle\langle 41\rangle} \frac{\Gamma\left(1+\alpha^{\prime} s\right) \Gamma\left(1+\alpha^{\prime} t-\alpha^{\prime} z\langle 13\rangle[23]\right)}{\Gamma\left(1+\alpha^{\prime} s+\alpha^{\prime} t-\alpha^{\prime} z\langle 13\rangle[23]\right)} .
$$

As a function of $z, \frac{A_{4}(z)}{z}$ has the following poles, apart from the one at $z=0$,

- at $z=-\frac{\langle 23\rangle}{\langle 13\rangle}$, with residue

$$
\operatorname{Res}\left(\frac{A_{4}(z)}{z}, z=-\frac{\langle 23\rangle}{\langle 13\rangle}\right)=-\frac{\langle 12\rangle^{4}}{\langle 12\rangle\langle 23\rangle\langle 34\rangle\langle 41\rangle} .
$$

This pole corresponds to the exchange of a gluon in the pinched disk diagram picture, and the residue yields exactly the Yang-Mills result. The latter fact is entirely expected since the residue must factor into two 3-gluon amplitudes; however, as these amplitudes receive no $\alpha^{\prime}$-corrections in superstring theory (cf. (2.2)), their product must reproduce the Yang-Mills result.

- at $z=\frac{k+\alpha^{\prime} t}{\alpha^{\prime}\langle 13\rangle[23]}, k \in \mathbb{N}$, with residues

$$
\operatorname{Res}\left(\frac{A_{4}(z)}{z}, z=\frac{k+\alpha^{\prime} t}{\alpha^{\prime}\langle 13\rangle[23]}\right)=\frac{(-1)^{k}}{k !} \frac{\langle 12\rangle^{4}}{\langle 12\rangle\langle 23\rangle\langle 34\rangle\langle 41\rangle} \frac{\left(\alpha^{\prime} s\right)\left(\alpha^{\prime} s-1\right) \cdots\left(\alpha^{\prime} s-k+1\right)}{t+k / \alpha^{\prime}} t .
$$

Summing the residues yields

$$
\begin{aligned}
\operatorname{Res}\left(\frac{A_{4}(z)}{z}, z=\right. & \left.-\frac{\langle 23\rangle}{\langle 13\rangle}\right)+\sum_{k=1}^{\infty} \operatorname{Res}\left(\frac{A_{4}(z)}{z}, z=\frac{k+\alpha^{\prime} t}{\alpha^{\prime}\langle 13\rangle[23]}\right) \\
& =-\frac{\langle 12\rangle^{4}}{\langle 12\rangle\langle 23\rangle\langle 34\rangle\langle 41\rangle} \frac{\Gamma\left(1+\alpha^{\prime} s\right) \Gamma\left(1+\alpha^{\prime} t\right)}{\Gamma\left(1+\alpha^{\prime} s+\alpha^{\prime} t\right)} .
\end{aligned}
$$

Using (5.27) and (5.4) this shows in particular

$$
\oint_{\infty} \frac{A_{4}(z)}{z} d z=0 .
$$




\section{The 'bad' shift}

Let us now consider the 'bad' shift for particles 2 and 3 (i.e. $p_{2}^{\dot{\alpha}} \rightarrow p_{2}^{\dot{\alpha}}-z p_{3}^{\dot{\alpha}} p_{3}^{\alpha} \rightarrow p_{3}^{\alpha}+z p_{2}^{\alpha}$ ) applied the same amplitude $A_{4}(++--)$. For this shift we obtain

$$
A_{4}(z)=(\langle 12\rangle+z\langle 13\rangle)^{2}([34]-z[24])^{2} \frac{\Gamma\left(\alpha^{\prime} s+z[12]\langle 13\rangle\right) \Gamma\left(\alpha^{\prime} t\right)}{\Gamma\left(1+\alpha^{\prime} t+\alpha^{\prime} s+z[12]\langle 13\rangle\right)} .
$$

In the same way as before the residue of the function $\frac{A_{4}(z)}{z}$ can be considered. In order to prove that the residue at infinity is absent directly, as we expect on the basis of our general argument, we should have

$$
A_{4}(0)=-\sum_{n=1}^{\infty} \frac{(-1)^{n}}{\alpha^{\prime 2} \Gamma(n+1)} \frac{n^{2}\left(n[23]+\alpha^{\prime}[12](\langle 12\rangle[24]+\langle 13\rangle[34])\right)^{2}}{\langle 13\rangle^{2}[12]^{4}} \frac{1}{n+s} \frac{\Gamma\left(\alpha^{\prime} t\right)}{\Gamma\left(1+\alpha^{\prime} t-n\right)} .
$$

That this is actually true can be verified explicitly by performing the sum using Mathematica. Note that an $\alpha^{\prime}$ expansion of the separate terms in the infinite sum is ill-defined as it involves the sum

$$
\sim \sum_{n=1}^{\infty} \frac{n^{2}}{\alpha^{2}}+\mathcal{O}\left(\frac{1}{\alpha^{\prime}}\right)
$$

which is a well-known diverging sum in string theory. This is related to the fact that according to the analysis above (specifically eq. (5.21) ), recursion should only work when

$$
\operatorname{Re}\left(3-\alpha^{\prime} t\right)<0 \text {. }
$$

Obviously, this condition cannot be satisfied when $\alpha^{\prime}=0$.

\section{Expansion in $\alpha^{\prime}$ within the recursion relation}

For the 'good' shift the equivalent condition (5.21) is met easily if $\alpha^{\prime}=0$ as the condition reads,

$$
\operatorname{Re}\left(-1-\alpha^{\prime} t\right)<0 .
$$

Noting that

$$
\text { st } \frac{\langle 12\rangle^{4}}{\langle 12\rangle\langle 23\rangle\langle 34\rangle\langle 41\rangle}=-\langle 12\rangle^{2}[34]^{2},
$$

one furthermore finds from (5.27) the form

$$
\begin{aligned}
A_{4}\left(1^{-},\right. & \left.2^{-}, 3^{+}, 4^{+}\right) \\
& =\frac{\langle 12\rangle^{4}}{\langle 12\rangle\langle 23\rangle\langle 34\rangle\langle 41\rangle}+\sum_{k=1}^{\infty} \frac{(-1)^{k-1}}{k !} \frac{\alpha^{\prime}\left(\alpha^{\prime} s-1\right) \cdots\left(\alpha^{\prime} s-k+1\right)}{t+k / \alpha^{\prime}}\langle 12\rangle^{2}[34]^{2} \\
& =\frac{\langle 12\rangle^{4}}{\langle 12\rangle\langle 23\rangle\langle 34\rangle\langle 41\rangle}+\sum_{k=1}^{\infty} \frac{1}{k} \frac{\alpha^{\prime}}{t+k / \alpha^{\prime}}\langle 12\rangle^{2}[34]^{2}+\cdots \\
& =\frac{\langle 12\rangle^{4}}{\langle 12\rangle\langle 23\rangle\langle 34\rangle\langle 41\rangle}+\frac{\pi^{2}}{6} \cdot \alpha^{\prime 2}\langle 12\rangle^{2}[34]^{2}+\mathcal{O}\left(\alpha^{\prime 3}\right)
\end{aligned}
$$


where the '.. ' in 5.43 refer to an $\alpha^{\prime}$ expansion of the numerator in the sum over $k$ in (5.42). We note from (5.43) that at each mass level $k$ the $\mathcal{O}\left(\alpha^{\prime 2}\right)$ correction takes the universal form $\frac{\alpha^{\prime 2}\langle 12\rangle^{2}[34]^{2}}{k^{2}}$. Furthermore, this correction can be factorized into two 'effective vertices' $\frac{\alpha^{\prime}\left(p_{1 \alpha} p_{4 \dot{\alpha}}\right)\left(p_{1 \beta} p_{4 \dot{\beta}}\right)}{k}$ and $\frac{\alpha^{\prime}\left(p_{2 \alpha} p_{3 \dot{\alpha}}\right)\left(p_{2 \beta} p_{3 \dot{\beta}}\right)}{k}$, each corresponding to a coupling between two gluons and a state with mass squared $k / \alpha^{\prime}$. This suggests in principle that one can compute higher-order corrections in $\alpha^{\prime}$ by use of further effective vertices. However, in practice this is not feasible, partly because these vertices become increasingly complicated, and partly because at a given order in $\alpha^{\prime}$ one needs to sum over several such vertices, cf. eq. (5.42).

The example of the four-point gluon amplitude also illustrates a general remark we would like to make about $\alpha^{\prime}$ expansion versus recursion relations. As already noted above, on physical grounds one expects that one should be careful about 'commuting' them. Above we explicitly see how this expectation works in a particular example. Based on this, we strongly suspect that for massless particles deriving recursion relations and applying $\alpha^{\prime}$ expansion commute only for what in field theory are 'well-behaved' shifts (see the discussion below eq. (5.30)). Specifically, we expect that when one derives recursion relations for amplitudes involving only massless particles, only a constraint on the kinematic variables of the form (5.40) will be encountered for a BCFW shift that works in field theory and 5.39) for a shift that does not work in field theory.

\section{The 5-gluon amplitude}

We now apply the same procedure to the more complicated case of the 5-gluon MHV amplitude which is given by

$$
A_{5}\left(1^{-}, 2^{-}, 3^{+}, 4^{+}, 5^{+}\right)=\frac{\langle 12\rangle^{4}}{\langle 12\rangle\langle 23\rangle\langle 34\rangle\langle 45\rangle\langle 51\rangle}\left(\alpha^{\prime 2} s_{51} s_{23} f_{1}+\alpha^{\prime 2}[12]\langle 23\rangle[35]\langle 51\rangle f_{2}\right),
$$

where

$$
f_{1}=\frac{\Gamma\left(\alpha^{\prime} s_{23}\right) \Gamma\left(\alpha^{\prime} s_{51}\right) \Gamma\left(\alpha^{\prime} s_{34}+1\right) \Gamma\left(\alpha^{\prime} s_{45}+1\right)}{\Gamma\left(\alpha^{\prime} s_{23}+\alpha^{\prime} s_{34}+1\right) \Gamma\left(\alpha^{\prime} s_{51}+\alpha^{\prime} s_{45}+1\right)}{ }_{3} F_{2}\left[\begin{array}{c}
\alpha^{\prime} s_{23}, \alpha^{\prime} s_{51},-\alpha^{\prime} s_{35} \\
\alpha^{\prime} s_{23}+\alpha^{\prime} s_{34}+1, \alpha^{\prime} s_{51}+\alpha^{\prime} s_{45}+1
\end{array} ; 1\right],
$$

and

$$
f_{2}=\frac{\Gamma\left(\alpha^{\prime} s_{23}+1\right) \Gamma\left(\alpha^{\prime} s_{51}+1\right) \Gamma\left(\alpha^{\prime} s_{34}+1\right) \Gamma\left(\alpha^{\prime} s_{45}+1\right)}{\Gamma\left(\alpha^{\prime} s_{23}+\alpha^{\prime} s_{34}+2\right) \Gamma\left(\alpha^{\prime} s_{51}+\alpha^{\prime} s_{45}+2\right)}{ }_{3} F_{2}\left[\begin{array}{c}
\alpha^{\prime} s_{23}+1, \alpha^{\prime} s_{51}+1,-\alpha^{\prime} s_{35}+1 \\
\alpha^{\prime} s_{23}+\alpha^{\prime} s_{34}+2, \alpha^{\prime} s_{51}+\alpha^{\prime} s_{45}+2
\end{array} ; 1\right] .
$$

In these equations $s_{i j}=\left(p_{i}+p_{j}\right)^{2}$ and the hypergeometric function ${ }_{3} F_{2}$ is defined through its series expansion

$$
{ }_{3} F_{2}(a, b, c ; d, e ; z)=\sum_{k=0}^{\infty} \frac{(a)_{k}(b)_{k}(c)_{k}}{(d)_{k}(e)_{k}} \frac{z^{k}}{k !} \quad, \quad(x)_{k} \equiv \frac{\Gamma(x+k)}{\Gamma(k)} .
$$

We now apply the BCFW shift

$$
\left\{\begin{array}{l}
p_{1 \alpha} \longrightarrow p_{1 \alpha} \\
p_{1 \dot{\alpha}} \longrightarrow p_{1 \dot{\alpha}}+z p_{2 \dot{\alpha}} \\
p_{2 \alpha} \longrightarrow p_{2 \alpha}-z p_{1 \alpha} \\
p_{2 \dot{\alpha}} \longrightarrow p_{2 \dot{\alpha}}
\end{array}\right.
$$


to the expression above (see appendix $\mathrm{C}$ for further details). For this particular shift, it follows that the $z$-dependent parts of both $f_{1}$ and $f_{2}$ take the form

$$
\begin{aligned}
f_{1,2}(z) \sim & \frac{\Gamma\left(a+\kappa_{1} z\right) \Gamma\left(b+\kappa_{2} z\right)}{\Gamma\left(d+\kappa_{1} z\right) \Gamma\left(e+\kappa_{2} z\right)}{ }_{3} F_{2}\left(a+\kappa_{1} z, b+\kappa_{2} z, c ; d+\kappa_{1} z, e+\kappa_{2} z ; 1\right) \\
& =\sum_{k=0}^{\infty} \frac{\Gamma\left(a+k+\kappa_{1} z\right) \Gamma\left(b+k+\kappa_{2} z\right)}{\Gamma\left(d+k+\kappa_{1} z\right) \Gamma\left(e+k+\kappa_{2} z\right)} \frac{(c)_{k}}{k !} .
\end{aligned}
$$

From the expression for the analytically continued 5-gluon amplitude (C.1) it is clear that in order to show $\oint_{\infty} \frac{A_{5}(z)}{z} d z=0$ it is enough to show that $\oint_{\infty} \frac{f_{i}(z)}{z} d z=0$ and $\oint_{\infty} f_{i}(z) d z=0$ for $i=1,2$. The two cases are treated completely analogously, and we focus here on the latter one.

Thus we aim to show $\oint_{\infty} f_{i}(z) d z=0$. From (5.51) it suffices to show ${ }^{14}$

$$
\oint_{\infty} \frac{\Gamma\left(a+\kappa_{1} z\right) \Gamma\left(b+\kappa_{2} z\right)}{\Gamma\left(d+\kappa_{1} z\right) \Gamma\left(e+\kappa_{2} z\right)} d z=0 .
$$

Redefining $z^{\prime}=\kappa_{1} z$ and putting $\kappa \equiv \kappa_{2} / \kappa_{1}$, from Stirling's formula the integrand has the large $z^{\prime}$ behavior

$$
\frac{\Gamma\left(a+z^{\prime}\right) \Gamma\left(b+\kappa z^{\prime}\right)}{\Gamma\left(d+z^{\prime}\right) \Gamma\left(e+\kappa z^{\prime}\right)} \simeq\left(z^{\prime}\right)^{a-d}\left(\kappa z^{\prime}\right)^{b-e}\left[1+\mathcal{O}\left(z^{\prime-1}\right)\right],
$$

where the corrections are a Laurent series in $z^{\prime}$ and one needs to require $-\pi<\arg \left(z^{\prime}\right)<\pi$ and $-\pi<\arg \left(\kappa z^{\prime}\right)<\pi$. (In further detail, both for $f_{1}$ and $f_{2}$ one finds $a-d=-\alpha^{\prime} s_{34}-1$ and $b-e=-\alpha^{\prime} s_{45}-1$, while $\kappa=-\langle 51\rangle[25] /(\langle 13\rangle[32])$.) Assuming $\arg (\kappa) \neq \pi$ we may now compute the contour integral of the left hand side of (5.53) around infinity in the following way. Considering first the contour to be a circle of finite radius, the points corresponding to the angles $-\pi$ and $\pi-\arg (\kappa)$ divide the circle into two arcs. Without loss of generality take the angular range of the larger arc to be from $\pi-\arg (\kappa)$ to $-\pi$ (i.e. so that the arc is contained mainly in the upper half plane). The integral over this arc (with the endpoints excluded) in the large radius limit may then be computed using the Stirling approximation (5.53). To compute the integral over the remaining part of the circle (including the points $-\pi$ and $\pi-\arg (\kappa))$ we apply Euler's reflection identity to the integrand which then becomes

$$
\frac{\Gamma\left(1-d-z^{\prime}\right) \Gamma\left(1-e-\kappa z^{\prime}\right)}{\Gamma\left(1-a-z^{\prime}\right) \Gamma\left(1-b-\kappa z^{\prime}\right)} \frac{\sin \left[\pi\left(d+z^{\prime}\right)\right]}{\sin \left[\pi\left(a+z^{\prime}\right)\right]} \frac{\sin \left[\pi\left(e+\kappa z^{\prime}\right)\right]}{\sin \left[\pi\left(b+\kappa z^{\prime}\right)\right]} .
$$

The asymptotic behavior of this function is $\left(-z^{\prime}\right)^{a-d}\left(-\kappa z^{\prime}\right)^{b-e}$ when $-\pi<\arg \left(-z^{\prime}\right)<\pi$ and $-\pi<\arg \left(-\kappa z^{\prime}\right)<\pi$. One may choose the full contour in (5.52) as an arbitrarily large circle avoiding the zeroes of $\sin \left[\pi\left(a+z^{\prime}\right)\right]$ and $\sin \left[\pi\left(b+\kappa z^{\prime}\right)\right]$. On the arc of this circle running from $-\pi$ to $\pi-\arg (\kappa)$ the $\frac{\sin \left[\pi\left(d+z^{\prime}\right)\right]}{\sin \left[\pi\left(a+z^{\prime}\right)\right]} \frac{\sin \left[\pi\left(e+\kappa z^{\prime}\right)\right]}{\sin \left[\pi\left(b+\kappa z^{\prime}\right)\right]}$ factor is bounded, and the integral over the left hand side in (5.53) is thus numerically bounded by the integral $\oint\left|z^{\prime}\right|^{a-d}\left|\kappa z^{\prime}\right|^{b-e} d z^{\prime}$. If $\operatorname{Re}(\mathrm{a}+\mathrm{b}-\mathrm{d}-\mathrm{e}+1)<0$ holds, then the integrand tends to zero as the radius is taken to infinity. In the case under study this amounts to

$$
\operatorname{Re}\left(-1-\alpha^{\prime}\left(\mathrm{s}_{34}+\mathrm{s}_{45}\right)\right)<0
$$

\footnotetext{
${ }^{14}$ Note that in the following we assume the validity of $\sum_{k} \oint=\oint \sum_{k}$.
} 
This concludes the proof of (5.52). Moreover, as observed above for the four-point gluon amplitude, the $\alpha^{\prime} \rightarrow 0$ limit commutes nicely with the constraint just derived.

As a further illustration of the result above, we consider in appendix 9 the poles of $A_{5}(z)$ and their corresponding residues explicitly. A general analysis is quite involved so that we focus on the residues to next-to-leading order in $\alpha^{\prime}$. We explicitly show how to order $\alpha^{\prime 2}$ the residues of the finite poles reproduce the five-point amplitude, implying that the residue at infinity is vanishing up to $\mathcal{O}\left(\alpha^{\prime 3}\right)$. The computation above thus proves that this holds exactly.

It is noteworthy to realize that every 5-point string theory amplitude involves on general grounds hypergeometric functions ${ }_{3} F_{2}$. The crucial ingredient in the argument above is that the BCFW shift acts as in (5.53) on this function. Thus if given a particular 5-point string amplitude one is able to finds a BCFW shift that has this property then a $\mathrm{BCFW}$ recursion relation can be found. It would be interesting to study this further and consider higher point functions.

\subsection{An attempt at truncating the BCFW recursion relations}

Since by the above argument the residue at infinity vanishes, the 5-gluon amplitude will equal (minus) the sum over all the residues of $\frac{A_{5}(z)}{z}$. To make the recursion explicit, one would like to factorize the resulting expressions into amplitudes and a sum over polarizations. However, although unitarity in principle guarantees the existence of such a factorization, it is neither clear from the explicit forms of the residues how to extract the factors, nor whether the expressions for such factors would yield useful building blocks for recursion relations.

It would be very interesting to obtain recursion relations order by order in $\alpha^{\prime}$. Inspired by the discussion following (5.42)-(5.44) that the full 4-gluon string amplitude is obtained by summing effective vertices over massive states of the string, we consider first the following schematic recursion relation for the 4-gluon amplitude

$$
A_{4}\left(1^{h_{1}}, 2^{h_{2}}, 3^{h_{3}}, 4^{h_{4}}\right)=\sum_{r, h(r)} A_{3}\left(1^{h_{1}}, 2^{h_{2}}, P_{r}\right) \frac{1}{\left(k_{1}+k_{2}\right)^{2}+m_{r}^{2}} A_{3}\left(P_{r}, 3^{h_{3}}, 4^{h_{4}}\right)
$$

where we have used the same notation as in (5.5) for the sum over string theory states. We will now attempt to construct a truncation of the BCFW recursion relation for the 5-gluon MHV amplitude $A_{5}\left(1^{-}, 2^{-}, 3^{+}, 4^{+}, 5^{+}\right)$that would capture the $\alpha^{\prime}$ correction up to a certain order $k_{0}$. In agreement with the discussion in section 5.4, we draw the recursion relations schematically as in 3 . Note that in the first line we have to include an additional diagram compared to the usual Yang-Mills case (the rightmost diagram in the first line of figure 3). Of course, this diagram vanishes for $m_{2}=0$. We can apply recursion again using the appropriate generalization of $(5.56)$ to arrive at the last line of figure 3 .

For the first graph in the bottom line, the strategy is now to split the sum over $m_{1}, m_{2}$ into four contributions $m_{1}=m_{2}=0 ; m_{1}>0, m_{2}=0 ; m_{1}=0, m_{2}>0$ and $m_{1}>0, m_{2}>0$. The first contribution is just the Yang-Mills contribution; the sum over $m_{1}$ in the second contribution and the sum over $m_{2}$ in the third contribution can be performed 


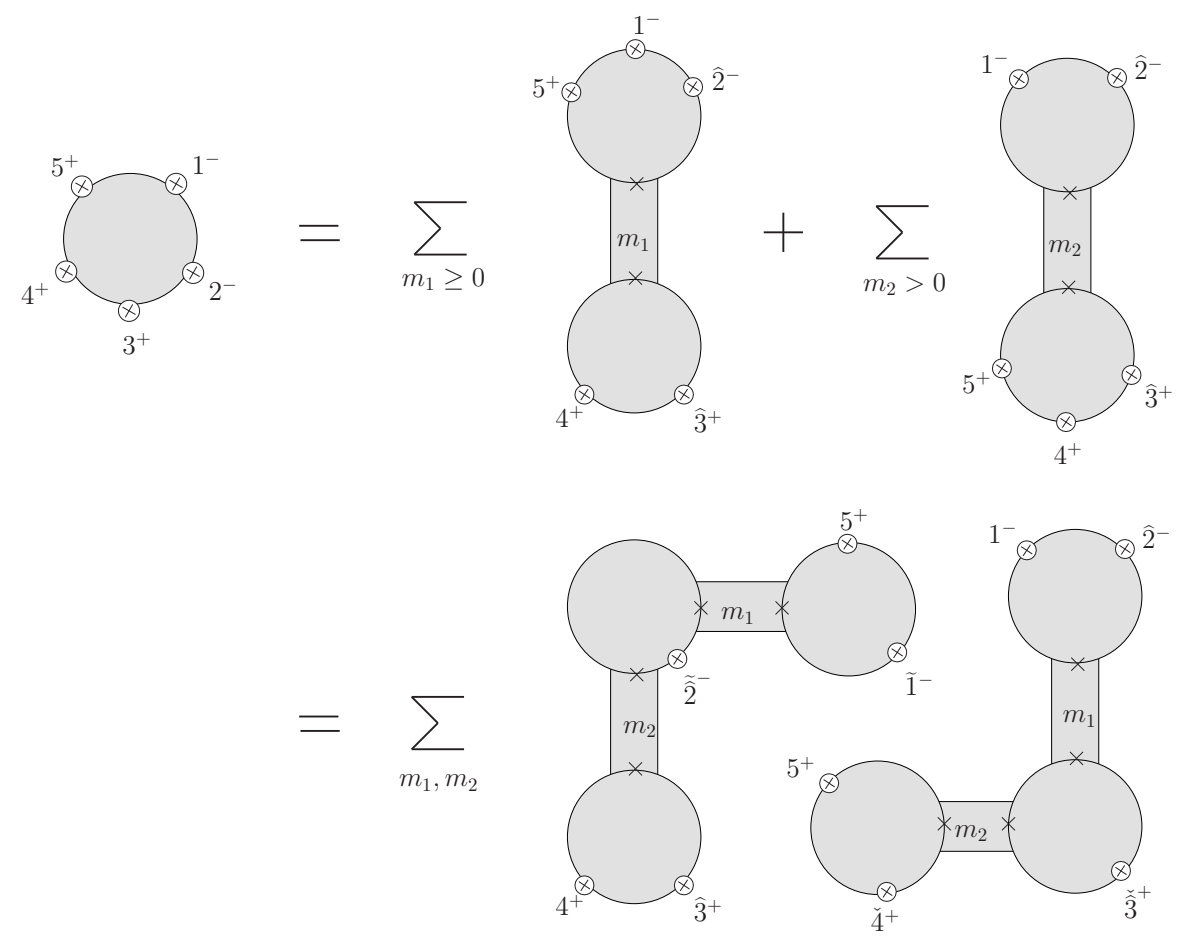

Figure 3: A graphical illustration of 5-gluon amplitude recursion diagrams

explicitly (equation (5.56)), yielding again 4-gluon amplitudes. Note that up to now we have only used known amplitudes involving gluons. Assuming for the moment that the fourth contribution (which involves amplitudes we have not encountered yet) starts appearing at an order in $\alpha^{\prime}$ larger than $k_{0}$, one can tentatively add the first three contributions and hope that this might reproduce the 5-gluon amplitude up to this order, thus providing a useful truncation of the recursion relations. Unfortunately, one finds discrepancies already at order $\alpha^{\prime 2}$ where only part of the $\alpha^{2}$ corrections (3.45) are reproduced, but interestingly no spurious poles.

The obvious origin of the discrepancy is the contribution from the sum over $m_{1}>$ $0, m_{2}>0$. However, that simply indicates that our truncation is too naive and new input is needed. There is a perhaps useful analogy here to one of the remarks at the end of section 3: soft and collinear limits only go some way to determine an amplitude as actual amplitudes are needed to fix some of the coefficients. For the $\alpha^{\prime 2}$ correction for instance, the 5-point amplitude is needed and once this is known, the amplitude is fixed completely to this particular order in $\alpha^{\prime}$ by the kinematic limits. Motivated by this observation, there is a suspicion that a similar truncation to the one studied in this subsection actually does work from 6-point amplitudes onwards (to order $\alpha^{\prime 2}$ ). This would be interesting to check.

\section{Conclusions and outlook}

In this article we have initiated a study of different approaches to calculating amplitudes 
in string theory motivated by recent developments in field theory. Using surprisingly basic considerations, three results have been established regarding string theory scattering, which will be commented on in turn. We subsequently turn to more general comments.

\subsection{MHV, CSW and BCFW:}

\section{MHV}

In section 3, we showed how the symmetry properties of the $\alpha^{\prime}$ corrected MHV amplitudes, and in particular their behavior under soft and collinear limits, severely restrict the unknown polynomials $Q_{m}^{(N)}$ that appear in the correction terms. In particular, we used a computer approach to find the general $\alpha^{\prime 3}$ correction explicitly for any number $N$ of external particles. It would of course be interesting to generalize this approach to higher orders. As commented upon earlier in this paper, for $\alpha^{4}$ this might still be possible using a more efficient computer approach along the lines of this paper (even though as we explained in the text, additional exact input from 7- and perhaps 8-point amplitudes is also required), but for higher orders one would need to further develop the analytic approach. These comments are further fleshed out at the end of chapter 3.

One comment which deserves some emphasis is that it would be useful to develop an idea of what the resulting polynomials would look like. To achieve this, the spinor notation is probably the most convenient. For example, using this notation, $Q_{2}^{(N)}$ can be written in the very simple form (3.45). It would be nice if one could find a similarly simple form for the polynomial $Q_{3}^{(N)}$ that we found in this paper, and use this to conjecture the general form of $Q_{m}^{(N)}$.

To round off this section, it would be extremely interesting to know if the full open superstring disk amplitude has localization properties on some natural object on twistor space or an extension thereof. Perhaps [9] can yield some insight here.

\section{CSW}

In section 4, scattering amplitudes have been derived for the Dirac-Born-Infeld action. Despite the fact that the action contains an infinite amount of vertices, it was shown that these conspire to produce very simple results. This needs to be understood more clearly, as the Rosly-Selivanov symmetry argument in [28] seems to suggest that the complete string theory should only generate helicity conserving amplitudes. This constraint might point the way to an all orders in $\alpha^{\prime}$ effective space-time action.

Concretely, it would be very interesting to see what happens to the 6-point MHV amplitude found by Stieberger and Taylor in [6] in the Abelian limit. The Rosly-Selivanov argument suggests that this amplitude, properly summed over all 120 contributions, actually might vanish completely if the disk level string theory amplitude indeed has a $U(1)$ duality symmetry. In turn, this would imply very strong constraints on the derivative corrections to the DBI action. Another potentially fruitful road to pursue would be to generalize the scalar action we have found in a way that naturally incorporates both Veneziano amplitudes as well as the DBI amplitudes. Perhaps here input from string field theory would be useful. 
The non-Abelian effective action remains less tractable, although the present article has yielded some more clues. Unfortunately it is still difficult to connect all the dots. Deriving scattering amplitudes from the known actions would still be interesting to check these actions against string theory amplitudes. Also the question of non-Abelian string theory CSW rules remains open, although our investigations in the Abelian case indicate that at some order in $\alpha^{\prime}$, modifications would be needed for NNMHV amplitudes at the least.

\section{BCFW}

In section 5, it was shown how in string theory BCFW recursion allows one to write higher point amplitudes in terms of on-shell lower point amplitudes. It was shown quite generally for 4-point string theory amplitudes how the residue at infinity which could have spoiled recursion is in fact absent. Since the Veneziano amplitude, for instance, not only diverges but has an essential singularity, this is perhaps surprising. On the other hand, intuition says that the behavior at infinity is related to the UV properties of the theory. Since the string has (exponentially) good UV properties, recursion is perhaps expected. From a purely theoretical point of view, the resulting recursion relations are fascinating as they allow one to reconstruct full amplitudes from their behavior at the boundary of a moduli space. We conjecture that the residue at infinity is absent for any open or closed string theory amplitude at the disk or sphere level in a flat background in any number of spacetime dimensions. In the language of [38], we therefore conjecture that 'tree-level' string theory is constructable.

On a more basic level however, there are several obstructions to be overcome to applying the recursion relations to obtain new amplitudes. In order to calculate in general with the recursion relations one needs to know all three-particle scattering amplitudes for all states in the string theory, and be able to do the spin sums and the sum over the full mass spectrum ${ }^{15}$. Although this problem is tractable in principle, in practice it seems to be very messy. We note for instance that a generic kinematical limit of the 5-gluon amplitude should, by unitarity, have as a residue a sum over polarizations of 2 sub-amplitudes. This factorization is however not manifest.

A more modest goal would be to be able to calculate amplitudes order by order in $\alpha^{\prime}$. This field theory expansion is not trivial however. This can be seen by the fact that most of the poles at which residues are evaluated move to infinity in this limit, leaving just the massless ones. This needs to be understood much more clearly, especially if one would like to derive the $\alpha^{\prime}$ corrections to the MHV amplitude for instance, perhaps by extending the final paragraph of section 5 to higher point amplitudes. On the other hand, $\alpha^{\prime}$ corrections to MHV amplitudes are an interesting sandbox to test out ideas as the answer is, with the results of this article, known up to $\alpha^{\prime 3}$.

Since the residue at infinity vanishes quite naturally for the BCFW two particle shift, it is natural to suspect that more general shifts also have good residue behavior. One

\footnotetext{
${ }^{15}$ See e.g. Refs. [39, 40] for results on three- and four-point superstring amplitudes involving massive string states at the first excited level.
} 
example of this is the shift studied in [34 for the one loop all-plus gluon amplitude in pure Yang-Mills theory. There a shift was found which displays good residue behavior for the known all-multiplicity expression for the amplitude. Since the $\alpha^{\prime 2}$ correction to the MHV amplitude is equivalent to this expression save a trivial ' $\langle i j\rangle^{4}$, prefactor, this will also obey the same recursion relation. However, starting from a known expression limits predictive power. It would still be interesting though to see if a similar procedure would work for our $\alpha^{\prime 3}$ result in section 3 .

\subsection{Field theory structures in string theory amplitudes}

We conclude with some more general comments and speculations about field theory structures in string theory amplitudes.

One other shift which could be interesting is the shift in Risager's derivation of the CSW rules [41]. Based on the experience with these rules in the Abelian case discussed in this paper in section 4.2, it would appear that these are not as straightforward as in ordinary field theory. However, there may very well be some sense to be made of them. It should be noted though that in field theory it is not straightforward to derive the vanishing of the appropriate residues directly [42], despite the fact that this is known to be true from an independent action-based derivation of the $\mathcal{N}=4 \mathrm{CSW}$ rules [23, 43] for all types of external particles.

As is well known, open string amplitudes are directly related to closed string amplitudes through the KLT relations [44]. Several results in this article therefore should have a direct interpretation in the closed string. For instance, one should be able to derive a form of $\alpha^{\prime}$ corrections of the MHV gravity amplitudes using these results.

The $\mathcal{N}=2$ string may have some role to play when it comes to elucidating underlying structures in string theory amplitudes ${ }^{16}$. This was also recently advocated in [9] with a different motivation. In field theory, the existence of MHV amplitudes and CSW rules for pure Yang-Mills theory in four dimensions is related to the existence of a gauge symmetry on twistor space which is not manifest from the space-time point of view [23]. In string theory, the Ward identity of ordinary gauge symmetry for on-shell amplitudes is a consequence of the world-sheet conformal symmetry. Extra gauge symmetry and its accompanying Ward identities should therefore somehow be connected to a larger conformal algebra, such as that for the $\mathcal{N}=2$ string. Since ordinary string theory can be embedded into this theory, this would be interesting to study further. Supporting evidence for this comes from the fact that the target space description of ordinary $\mathcal{N}=2$ string theory is self-dual Yang-Mills theory in $d=4$. As shown in 45, there is a direct connection here between the twistor space gauge symmetry and the $\mathcal{N}=2$ string.

It is interesting to note that the BCFW recursion relations, if extendable to the full string theory amplitudes as conjectured, basically state that all amplitudes can be constructed from the three-point functions and propagators. This is a recurring theme in string theory, which also can be found for example in open string field theory, conformal field theory and topological string theory. An interesting question to ask is whether the

\footnotetext{
${ }^{16}$ This suggestion was made to us by Shinji Hirano.
} 
connection to these results goes beyond an analogy. This is of course most natural in the case of open string field theory, on which we already commented in the main text. As for connections to conformal field theory and the topological string, in these theories, as in this case, it is often easy to split amplitudes into smaller parts, summed over some intermediate Hilbert space. The hard part is then to identify this Hilbert space with the space of physical states that one is interested in. (See e.g. section 3.3.2 of [46] for how this is done in the topological string case.) The crucial ingredient in such proofs lies is the presence of a BRST-like symmetry on the worldsheet. It would be interesting to see if a similar structure can be found in the case of the full-fledged open superstring theory amplitudes. In particular, since this argument is independent of the global topology of the world-sheet, it would imply that BCFW-like relations are not only valid at the disk level, as we argued in this paper, but also at higher loops. It is easy to speculate (and probably hard to prove if true at all) that the BRST-like symmetry mentioned here is connected to the larger gauge symmetry mentioned above for the $\mathcal{N}=2$ string.

As a final comment we would like to point out that most of the basic ideas used in this paper are actually close to 40 years old. In a sense, we simply advocate applying analytic S-matrix techniques to string theory amplitudes. Motivated by recent developments of S-matrix methods in field theory, we have added some new ingredients. These are the spinor helicity notation, the recent appreciation of the usefulness of complex momenta and a clear focus on calculating helicity amplitudes. As string theory has until recently been the great success of the original analytic S-matrix approach, it should perhaps not be too surprising that these new ingredients appear to be very fruitful to explore. In a sense, it would appear that string theory is the 'simplest quantum field theory' 447.

\section{Acknowledgments}

The authors gratefully acknowledge discussions with Paolo di Vecchia, Costas Zoubos, David Skinner and Shinji Hirano. The research of MV was supported by the South African Research Chairs Initiative of the Department of Science and Technology and National

Research Foundation. MV would like to thank the Niels Bohr Institute for kind hospitality during the initial stages of this project. The work of NO is partially supported by the European Community's Human Potential Programme under contract MRTN-CT-2004005104 'Constituents, fundamental forces and symmetries of the universe'. The research of RB was supported by a Marie Curie European Reintegration Grant within the 7th European Community Framework Programme. All figures in this paper except figure 6 were drawn using Jaxodraw [48].

\section{A. Technical details relevant for section 3}

\section{A.1 Cyclically reducible polynomials for scalars}

In this sub-appendix, we prove that for the cyclically reducible polynomials of degree $d$ in 
$N$ scalar variables, a basis is given by

$$
\left(a_{1} a_{2} \ldots a_{m}\right) \equiv \sum_{\left(i_{1}, \ldots i_{m}\right)} x_{i_{1}}^{a_{1}} \cdots x_{i_{m}}^{a_{m}}
$$

where $m \leq N, a_{i} \geq 1$ are positive integers with $\sum a_{i}=d$, the sum is over all cyclically ordered sequences $\left(i_{1}, \ldots i_{m}\right)$ of length $m$, and we only consider cyclically inequivalent sequences $\left(a_{1} \ldots a_{m}\right)$.

First of all, note that the above polynomials are indeed cyclically symmetric, and that their reductions after we set several variables to zero are again cyclically symmetric in the remaining variables. That is, they are all cyclically reducible. Moreover, every monomial of degree $d$ appears in one of the polynomials. Therefore, to show that these polynomials form a basis, we only have to show that they are the smallest possible ones that are cyclically reducible.

To see this, take two monomial terms $m_{1}^{(0)}, m_{2}^{(0)}$ out of one of the polynomials above. We now show inductively that indeed, if one of these terms appears in a cyclically reducible polynomial, so must the other.

- In case $m_{1}^{(0)}$ can be obtained from $m_{2}^{(0)}$ by a cyclic permutation of the indices, we are done.

- Otherwise, there must be a variable which does not appear in $m_{1}^{(0)}$, and one which does not appear in $m_{2}^{(0)}$. We now study the monomials $m_{1}^{(1)}, m_{2}^{(1)}$ obtained by cyclically permuting until these 'missing variables' have the maximum index $N$. Clearly, $m_{i}^{(1)}$ must be in the same basis polynomial as $m_{i}^{(0)}$. Now, we take a soft limit on the $N^{\prime}$ th variable in $m_{i}^{(1)}$, and go back to step one.

As an example, let us study

$$
m_{1}^{(0)}=x_{3}^{2} x_{4} x_{5}, \quad m_{2}^{(0)}=x_{1}^{2} x_{3} x_{5} .
$$

By cyclic permutation in all five variables, the basis polynomial that contains $m_{i}^{(0)}$ must also contain $m_{i}^{(1)}$, where

$$
m_{1}^{(1)}=x_{1}^{2} x_{2} x_{3}, \quad m_{2}^{(1)}=x_{2}^{2} x_{4} x_{1} .
$$

Note that we have arranged these monomials in such a way that $x_{5}$ is missing. When we take the soft limit in $x_{5}$, we see after a cyclic permutation in the remaining four variables of $m_{2}$ that these basis polynomials must also contain

$$
m_{1}^{(2)}=x_{1}^{2} x_{2} x_{3}, \quad m_{2}^{(2)}=x_{3}^{2} x_{1} x_{2} .
$$

Now we take the soft limit in $x_{4}$, after which the remaining monomials are cyclic permutations of each other. Hence, it is clear that $m_{1}^{(2)}$ and $m_{2}^{(2)}$ must be in the same basis polynomial, and therefore, reasoning backwards, that $m_{1}^{(0)}$ and $m_{2}^{(0)}$ were in the same one. It is easy to see that this procedure will relate any two monomials that appear in the polynomials we constructed above, and hence these indeed form a basis of all cyclically reducible polynomials. 


\section{A.2 Combinatorics of necklaces}

In section 3.2, we encountered the problem of counting the number $N_{d}$ of necklaces

$$
\left(a_{1} \ldots a_{m}\right)
$$

with the constraints $a_{i} \geq 1, \sum a_{i}=d$. Here, the fact that the sequence is a 'necklace' means that we count sequences only once if they differ by a cyclic permutation.

The trick to counting these necklaces is to rewrite them as follows: instead of the number $a_{i}$, write a one followed by $\left(a_{i}-1\right)$ zeroes. So, for example, we would rewrite

$$
(3115) \mapsto(1001110000)
$$

A little thought shows that this map is injective onto the set of 'binary' necklaces of length $d$. Furthermore, it is nearly surjective: the only necklace that is not an image under this map is the necklace consisting of only zeroes. Therefore, our problem is solved if we can count binary necklaces of length $d$, where we have to remember to subtract 1 in the end for the zero necklace.

The latter problem is much easier to solve. Note that there are $2^{d}$ binary sequences of length $d$. If a necklace has no symmetry properties, it appears exactly $d$ times (once for each cyclic permutation) in the set of binary sequences. So, as a first approximation, we might take

$$
N_{d} \sim \frac{2^{d}}{d}
$$

However, a necklace which consists of a number of copies of a smaller sequence is undercounted in this way; for example, the necklace

$$
(1000010000)
$$

appears only five times in the set of all binary sequences of length ten, not ten times. Thus, for every divisor $d_{i}$ of $d$, we have to compensate for undercounting of the number of repeated sequences of length $d_{i}$. Doing this carefully, we find the full answer,

$$
N_{d}=-1+\frac{1}{d} \sum_{d_{i} \mid d} \phi\left(d_{i}\right) 2^{d / d_{i}} .
$$

Here, $\phi\left(d_{i}\right)$ is the number of integers $k \leq d_{i}$ that are relatively prime to $d_{i}$. This number is known as Euler's totient function, and can be written as

$$
\phi\left(d_{i}\right)=d_{i} \prod_{p \mid d_{i}}\left(1-p^{-1}\right),
$$

where the product is over all different primes $p$ dividing $d_{i}$.

\section{A.3 Collinearity of $Q_{3}^{(N)}$}

In this sub-appendix, we show that the expression in the curly brackets of equation (3.51) vanishes after using momentum conservation and Schouten identities, thus proving that $Q_{3}^{(N)}$ in equation (3.47) reduces to $Q_{3}^{(N-1)}$ in the collinear limit. 


\section{Terms with metric contractions only}

Let us begin with the terms involving only metric contractions. Up to a scale factor, these read

$$
\begin{aligned}
T_{1}= & 3\left(s_{13} s_{2 x} s_{4 x}\right)+3\left(s_{12} s_{1 x} s_{3 x}\right)+6\left(s_{23} s_{1 x} s_{4 x}\right) \\
& +3\left(s_{23} s_{1 x} s_{3 x}\right)+3\left(s_{24} s_{1 x} s_{3 x}\right)+\left(s_{1 x} s_{1 x} s_{1 x}\right),
\end{aligned}
$$

where we use the notation that was introduced below (3.49). In order to show that this vanishes, let us begin by writing down the momentum conservation relation

$$
\begin{aligned}
0=\sum_{i}\left(s_{i 2} s_{1 x} s_{3 x}\right)= & \left(s_{13} s_{2 x} s_{4 x}\right)+\left(s_{12} s_{1 x} s_{3 x}\right)+2\left(s_{23} s_{1 x} s_{4 x}\right) \\
& +\left(s_{23} s_{1 x} s_{3 x}\right)+\left(s_{24} s_{1 x} s_{3 x}\right)+\left(s_{2 x} s_{1 x} s_{3 x}\right) .
\end{aligned}
$$

The consecutive terms in this expression arise from the terms where the index $i$ is smaller

than the index represented by 1 , equal to it, between those represented by 1 and 2 , and so on. Subtracting this equation three times from (A.11) we see that the expression for $T_{1}$ simplifies to

$$
T_{1}=\left(s_{1 x} s_{1 x} s_{1 x}\right)-3\left(s_{1 x} s_{2 x} s_{3 x}\right) .
$$

Next, we use the fact that

$$
\sum_{i} s_{i x}=\left(s_{1 x}\right)=0
$$

Taking the cube of this expression, we find

$$
0=\left(s_{1 x}\right)^{3}=\left(s_{1 x} s_{1 x} s_{1 x}\right)+3\left(s_{1 x} s_{1 x} s_{2 x}\right)+3\left(s_{1 x} s_{2 x} s_{2 x}\right)+6\left(s_{1 x} s_{2 x} s_{3 x}\right) .
$$

On the other hand, multiplying (A.14 with $\left(s_{1 x} s_{2 x}\right)$ gives

$$
0=\left(s_{1 x}\right)\left(s_{1 x} s_{2 x}\right)=\left(s_{1 x} s_{1 x} s_{2 x}\right)+\left(s_{1 x} s_{2 x} s_{2 x}\right)+3\left(s_{1 x} s_{2 x} s_{3 x}\right) .
$$

Subtracting this last equation three times from equation (A.15), we find back the expression (A.13), so we have shown that

$$
T_{1}=0
$$

completing the proof for the terms with metric contractions only.

\section{Terms with $\epsilon$ - and metric contractions}

Next, we study the terms which involve both $\epsilon$ - and metric contractions. These are proportional to

$$
T_{2}=\left(\epsilon_{x 123} s_{2 x}\right)-2\left(\epsilon_{234 x} s_{1 x}\right)+2\left(\epsilon_{123 x} s_{3 x}\right)+2\left(\epsilon_{134 x} s_{2 x}\right)+4\left(\epsilon_{123 x} s_{4 x}\right) .
$$

We begin by comparing this to the following Schouten identity, where we antisymmetrize over the first five indices:

$$
\left(\epsilon_{123 x} s_{4 x}\right)-\left(\epsilon_{234 x} s_{1 x}\right)+\left(\epsilon_{134 x} s_{2 x}\right)-\left(\epsilon_{124 x} s_{3 x}\right)=0 .
$$


Subtracting this twice from $T_{2}$, we are left with

$$
T_{2}=\left(\epsilon_{x 123} s_{2 x}\right)+2\left(\epsilon_{123 x} s_{3 x}\right)+2\left(\epsilon_{123 x} s_{4 x}\right)+2\left(\epsilon_{124 x} s_{3 x}\right) .
$$

Now, we use the momentum conservation result

$$
0=\sum_{i}\left(\epsilon_{i 12 x} s_{3 x}\right)=\left(\epsilon_{123 x} s_{4 x}\right)+\left(\epsilon_{123 x} s_{3 x}\right)+\left(\epsilon_{124 x} s_{3 x}\right)
$$

to reduce this to

$$
T_{2}=\left(\epsilon_{x 123} s_{2 x}\right) .
$$

Finally, we see that this remaining term vanishes by noticing that it equals

$$
0=\sum_{i}\left(\epsilon_{i 12 x} s_{2 x}\right)=\left(\epsilon_{123 x} s_{2 x}\right)
$$

Thus, we have found that also

$$
T_{2}=0
$$

and hence the entire polynomial in curly brackets in (3.51) vanishes.

\section{A.4 Counting Lorentz invariant polynomials}

In this sub-appendix we count the number of Lorentz-invariants modulo Schouten identities in four dimensions which can be constructed out of $d$ vectors $p_{i}$. To avoid complicated expressions involving the 4-dimensional epsilon tensor, we will work in spinor notation. In this notation both epsilon tensor and metric can be expressed in terms of $\epsilon_{\alpha \beta}$ and the counting problem reduces to the number of independent tensors $I(\epsilon)_{\alpha_{1} \ldots \alpha_{d}, \dot{\alpha}_{1} \ldots \dot{\alpha}_{d}}$. Hence we can split up the problem into dotted and undotted indices. Since these obviously transform as a tensor product of the spinor representation, one way to state the counting problem is to count the number of independent invariants (i.e. the (1) representation of $S U(2)$ ) in the representation

$$
\underbrace{(2) \otimes(2) \otimes \ldots \otimes(2)}_{d} .
$$

In particular, we find

$$
\begin{array}{ll}
d & \text { reps } \\
2 & (1) \oplus(3) \\
4 & 2(1) \oplus 2(3) \oplus(5) \\
6 & 5(1) \oplus 9(3) \oplus 5(5) \oplus(7) \\
8 & 14(1) \oplus 28(3) \oplus 20(5) \oplus 7(7) \oplus(8) .
\end{array}
$$

Based on these numerics, Sloane's wonderful encyclopedia 25] suggests Catalan's numbers (A000108) for the number of trivial representation in the tensor product (A.25). This can be seen to be true by using one of the there suggested interpretations of these numbers. For this, one should realize Schouten identities are incorporated in the above counting. 


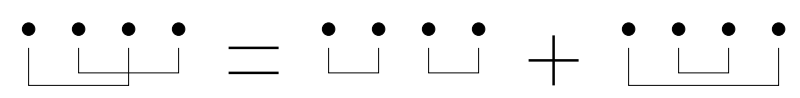

Figure 4: Illustration of the Schouten identity in 2 dimensions. Dots represent indices, brackets represent contraction of the indices by the epsilon tensor.

For $d=4$ for instance, naive counting yields 3 invariants, one of which is the sum of the others by the Schouten identity in two dimensions. More generally, this can be illustrated by considering the construction of the actual invariants. Since pairs of indices will be contracted for this, one can denote a particular contraction by drawing lines from one to the other position. In this notation, it is easy to see that the Schouten identity can be drawn schematically as in figure (41) for any four indices. Hence the counting problem can be reduced to the problem of connecting $d$ dots by non-intersecting pair-wise arcs. The number of these is given by the Catalan number $c_{\frac{d}{2}}{ }^{17}$. Having counted the number of spinor contractions for one kind of spinor, the number of independent Lorentz invariants follows by squaring.

\section{B. On-shell recursion relations for the Dirac-Born-Infeld action}

In section 4 we managed to derive a significantly simpler version of perturbation theory for tree level helicity amplitudes derived from the Dirac-Born-Infeld action in four dimensions. Furthermore, in section 5 we studied on-shell recursion relations. It is therefore natural to ask if the DBI perturbation theory can be used to study some form of on-shell recursion relations for these amplitudes. This will be discussed in this appendix.

The simplest example is the 4-point function. It is quite obvious that shifting in any way will immediately lead to residues at infinity. Although this can be cured, one could first assume knowledge of the 4-point function and see if the 6-point function can be calculated from this. Therefore, let us study the 6-point NMHV amplitude 4.19). It is natural to try to shift a positive and a negative helicity photon, since one can choose then a shift which lets the scalar-to-field strengths couplings invariant. Let us choose these to be the 3rd and 4th particle respectively and study the amplitude as a contour integral as in section 5 ,

$$
A_{6}^{\mathrm{DBI}}\left(1^{+} 2^{+} 3^{+} 4^{-} 5^{-} 6^{-}\right)(0)=\oint_{z=0} \frac{A_{6}^{\mathrm{DBI}}\left(\ldots \hat{3}^{+} \hat{4}^{-} \ldots\right)(z)}{z} d z,
$$

where the hatted indices denote the shifted momenta. Again, we choose the shift for which $\hat{3}_{\dot{\alpha}}=3_{\dot{\alpha}}$ and $\hat{4}_{\alpha}=4_{\alpha}$. Pulling the contour to infinity yields a would-be BCFW recursion relation. However, one can already see by studying the explicit form of (4.19) that there are poles in the amplitude where both shifted momenta appear in the denominator, hence leading to a $z$-independent part of the amplitude which in turn leads to poles at infinity. Postponing this point to slightly later, let us calculate one of the finite terms where for instance particles 3,5,6 appear on one side of the recursion,

$$
\operatorname{Res}\left(\frac{A_{6}(z)}{z}, z=\frac{1}{[4|(1+2)| 3\rangle}\left(p_{1}+p_{2}+p_{3}\right)^{2}\right)=\frac{\pi^{4} \alpha^{\prime 4}}{4} \frac{\langle 12\rangle^{2}[4 \hat{\imath}]^{2}\langle\hat{\imath} 3\rangle^{2}[56]^{2}}{\left(p_{1}+p_{2}+p_{4}\right)^{2}} \text {. }
$$

\footnotetext{
${ }^{17}$ for some visuals, see for instance http://www.maths.usyd.edu.au/u/kooc/catalan/cat2smi.pdf
} 

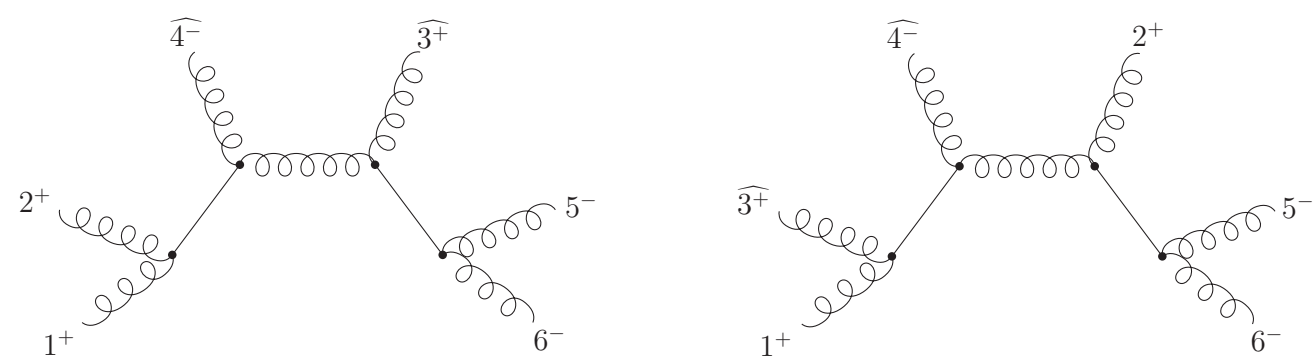

Figure 5: Examples of 'good' (left) and 'bad' (right) terms in the direct, naive application of BCFW recursion relations for Abelian superstring amplitudes.

Here the intermediate, on-shell momentum is

$$
\hat{\imath}_{\alpha} \hat{\imath}_{\dot{\alpha}}=p_{1}+p_{2}+\hat{p}_{4}
$$

At this point we can use the standard relation,

$$
[4 \hat{\imath}]\langle\hat{\imath} 3\rangle=[4|i| 3\rangle,
$$

to see that this particular term has the right form to be one of the terms of the expression of the full amplitude 4.19) .

To see where the missing terms could arise, note that the 'good' and 'bad' terms can be drawn by the two example contributions in figure 5: the left figure shows a diagram which has an explicit kinematic pole from the photon exchange, the other diagram does not since the scalar propagator is trivial. If this propagator would be non-trivial, i.e. contained a standard momentum factor, then the troublesome terms would also have a pole at finite values of $z$ (where massive particles go on-shell) and contribute. Actually, one can promote the scalar to a propagating field and then expand the resulting expression in $\alpha^{\prime}$, keeping only the lowest order term. The trick to circumvent the problem of the 'bad' amplitudes is to first derive the recursion relations and then expand in $\alpha^{\prime}$. This is of course in line with general remarks about the connection between residues at infinity and the UV behavior of the theory under study in section 5 .

Residues of the shifted amplitudes involving the poles of the now-propagating scalar fields simply reduce to the right expressions for our particular choice of shift, including kinematic poles since the rest of the BCFW procedure is then simply a self-similarity transformation. For instance, to obtain the contribution to the scattering amplitude with a pole in the channel (256) depicted in figure 5 one studies the pole of the scattering amplitude for which

$$
\left(\hat{p}_{3}+p_{1}\right)^{2} \rightarrow-1 / \alpha^{\prime}
$$

and hence

$$
z=\frac{1}{[4|1| 3\rangle}\left(\frac{1}{\alpha^{\prime}}+\left(p_{3}+p_{1}\right)^{2}\right) .
$$


Note that this pole migrates to infinity if $\alpha^{\prime} \rightarrow 0$. With this simple observation we obtain for this specific contribution

$$
\operatorname{Res}\left(\frac{A(z)}{z}, z=\frac{1}{[4|1| 3\rangle}\left(\frac{1}{\alpha^{\prime}}+\left(p_{3}+p_{1}\right)^{2}\right)\right)=\frac{1}{\left(p_{1}+p_{3}\right)^{2}+\frac{1}{\alpha^{\prime}}} A\left(\hat{3}^{+} 1^{+} \hat{k}\right) A\left(\hat{\bar{k}} \hat{4}^{-} 2^{+} 5^{-} 6^{-}\right),
$$

where $k$ is the now-propagating scalar field with momentum $\hat{p}_{3}+p_{1}$. As observed before, the hats can simply be removed directly for the shift under study, while the prefactor can conveniently be expanded in terms of $\alpha^{\prime}$. This is easily seen to generate one of the missing contributions of the six-point amplitude in (4.19). Actually, it is diagrammatically equivalent to one of the Feynman diagrams generated by (4.30). It is very important though to remember that only the leading part in $\alpha^{\prime}$ of the above construction actually reproduces the string theory amplitude.

With these extra propagators, it is straightforward to see that since both a negative and a positive helicity gluon are shifted, these shifts will never be on the same vertex leading into the diagram. Therefore, in any expression for the amplitudes derived from the Feynman rules there are always suppressive powers of $z$, while for this shift there are no compensating powers of $z$ in the numerator. Therefore, the residue at infinity of this integral will always vanish, proving the validity of BCFW recursion formulas in this case.

\section{Poles and residues for the five-point gluon amplitude}

In this appendix we provide further details of applying the BCFW shift (5.49) to the 5gluon MHV amplitude (5.45). First, one finds $s_{23} \longrightarrow s_{23}-z\langle 13\rangle[32], s_{51} \longrightarrow s_{51}+z\langle 51\rangle[25]$ and $\langle 23\rangle \longrightarrow\langle 23\rangle-z\langle 13\rangle$. Thus, the analytically continued amplitude is

$$
A_{5}(z)=\frac{\langle 12\rangle^{4}}{\langle 12\rangle\langle 23\rangle\langle 34\rangle\langle 45\rangle\langle 51\rangle}\left(\alpha^{\prime 2}\left(s_{51}+z\langle 51\rangle[25]\right) s_{23} f_{1}(z)+\alpha^{\prime 2}[12]\langle 23\rangle[35]\langle 51\rangle f_{2}(z)\right),
$$

where

$$
\begin{gathered}
f_{1}(z)=\frac{\Gamma\left(\alpha^{\prime} s_{23}-\alpha^{\prime} z\langle 13\rangle[32]\right) \Gamma\left(\alpha^{\prime} s_{51}+\alpha^{\prime} z\langle 51\rangle[25]\right) \Gamma\left(\alpha^{\prime} s_{34}+1\right) \Gamma\left(\alpha^{\prime} s_{45}+1\right)}{\Gamma\left(\alpha^{\prime} s_{23}+\alpha^{\prime} s_{34}-\alpha^{\prime} z\langle 13\rangle[32]+1\right) \Gamma\left(\alpha^{\prime} s_{51}+\alpha^{\prime} s_{45}+\alpha^{\prime} z\langle 51\rangle[25]+1\right)} \\
\times{ }_{3} F_{2}\left[\begin{array}{c}
\alpha^{\prime} s_{23}-\alpha^{\prime} z\langle 13\rangle[32], \alpha^{\prime} s_{51}+\alpha^{\prime} z\langle 51\rangle[25],-\alpha^{\prime} s_{35} \\
\alpha^{\prime} s_{23}+\alpha^{\prime} s_{34}-\alpha^{\prime} z\langle 13\rangle[32]+1, \alpha^{\prime} s_{51}+\alpha^{\prime} s_{45}+\alpha^{\prime} z\langle 51\rangle[25]+1
\end{array} ; 1\right],
\end{gathered}
$$

and

$$
\begin{gathered}
f_{2}(z)=\frac{\Gamma\left(\alpha^{\prime} s_{23}-\alpha^{\prime} z\langle 13\rangle[32]+1\right) \Gamma\left(\alpha^{\prime} s_{51}+\alpha^{\prime} z\langle 51\rangle[25]+1\right) \Gamma\left(\alpha^{\prime} s_{34}+1\right) \Gamma\left(\alpha^{\prime} s_{45}+1\right)}{\Gamma\left(\alpha^{\prime} s_{23}+\alpha^{\prime} s_{34}-\alpha^{\prime} z\langle 13\rangle[32]+2\right) \Gamma\left(\alpha^{\prime} s_{51}+\alpha^{\prime} s_{45}+\alpha^{\prime} z\langle 51\rangle[25]+2\right)} \\
\times{ }_{3} F_{2}\left[\begin{array}{c}
\alpha^{\prime} s_{23}-\alpha^{\prime} z\langle 13\rangle[32]+1, \alpha^{\prime} s_{51}+\alpha^{\prime} z\langle 51\rangle[25]+1,-\alpha^{\prime} s_{35}+1 \\
\alpha^{\prime} s_{23}+\alpha^{\prime} s_{34}-\alpha^{\prime} z\langle 13\rangle[32]+2, \alpha^{\prime} s_{51}+\alpha^{\prime} s_{45}+\alpha^{\prime} z\langle 51\rangle[25]+2
\end{array} ; 1\right] .
\end{gathered}
$$

As a function of $z, \frac{A_{5}(z)}{z}$ has the following poles and corresponding residues:

- at $z=\frac{\langle 23\rangle}{\langle 13\rangle}$, with residue

$$
\begin{aligned}
\operatorname{Res}\left(\frac{A_{5}(z)}{z}, z=\frac{\langle 23\rangle}{\langle 13\rangle}\right) & \\
= & -\frac{\langle 12\rangle^{4}}{\langle 12\rangle\langle 23\rangle\langle 34\rangle\langle 45\rangle\langle 51\rangle} \frac{\Gamma\left(\alpha^{\prime} s_{51}+\alpha^{\prime} \frac{\langle 23\rangle\langle 51\rangle[25]}{\langle 13\rangle}+1\right) \Gamma\left(\alpha^{\prime} s_{45}+1\right)}{\Gamma\left(\alpha^{\prime} s_{51}+\alpha^{\prime} s_{45}+\alpha^{\prime} \frac{\langle 23\rangle\langle 51\rangle[25]}{\langle 13\rangle}+1\right)} \\
= & -\frac{\langle 12\rangle^{4}}{\langle 12\rangle\langle 23\rangle\langle 34\rangle\langle 45\rangle\langle 51\rangle}\left[1-\frac{\pi^{2} \alpha^{\prime 2}}{6} s_{45}\left(s_{51}+\frac{\langle 23\rangle\langle 51\rangle[25]}{\langle 13\rangle}\right)+\mathcal{O}\left(\alpha^{\prime 3}\right)\right] .
\end{aligned}
$$


As opposed to the 4-gluon case (5.32) this does not reproduce the Yang-Mills result. Of course, this follows simply from the fact that on this pole the amplitude factorizes into a 3-gluon amplitude and a 4-gluon amplitude (as can be seen directly in (C.4)), where the latter amplitude receives $\alpha^{\prime}$-corrections in superstring theory.

- at $z=\frac{\alpha^{\prime} s_{23}+k_{1}}{\alpha^{\prime}\langle 13\rangle[32]}, k_{1} \in \mathbb{N}$, with residue

$$
\begin{aligned}
& \operatorname{Res}\left(\frac{A_{5}(z)}{z}, z=\frac{\alpha^{\prime} s_{23}+k_{1}}{\alpha^{\prime}\langle 13\rangle\langle 32]}\right) \\
& =\frac{\langle 12\rangle^{4}}{\langle 12\rangle\langle 23\rangle\langle 34\rangle\langle 45\rangle\langle 51\rangle}\left[\alpha^{\prime 2}\left(s_{51}+\left(s_{23}+\frac{k_{1}}{\alpha^{\prime}}\right) \frac{\langle 51\rangle[25]}{\langle 13\rangle\langle 32]}\right) s_{23} \widetilde{f}_{1}+\alpha^{\prime 2}[12]\langle 23\rangle[35]\langle 51\rangle \widetilde{f}_{2}\right],
\end{aligned}
$$

where

$$
\begin{aligned}
\widetilde{f}_{1}= & \frac{(-1)^{k_{1}+1}}{k_{1} !} \frac{1}{\alpha^{\prime} s_{23}+k_{1}} \frac{\Gamma\left(\alpha^{\prime} s_{51}+\left(\alpha^{\prime} s_{23}+k_{1}\right) \frac{\langle 51\rangle[25]}{\langle 13\rangle\langle 32]}\right) \Gamma\left(\alpha^{\prime} s_{34}+1\right) \Gamma\left(\alpha^{\prime} s_{45}+1\right)}{\Gamma\left(-k_{1}+1+\alpha^{\prime} s_{34}\right) \Gamma\left(\alpha^{\prime} s_{51}+\alpha^{\prime} s_{45}+\left(\alpha^{\prime} s_{23}+k_{1}\right) \frac{\langle 51\rangle\langle 25]}{\langle 13\rangle\langle[32]}+1\right)} \\
& \times{ }_{3} F_{2}\left[\begin{array}{c}
-k_{1}, \alpha^{\prime} s_{51}+\left(\alpha^{\prime} s_{23}+k_{1}\right) \frac{\langle 51\rangle[25]}{\langle 13\rangle[32]},-\alpha^{\prime} s_{35} \\
-k_{1}+1+\alpha^{\prime} s_{34}, \alpha^{\prime} s_{51}+\alpha^{\prime} s_{45}+\left(\alpha^{\prime} s_{23}+k_{1}\right) \frac{\langle 51\rangle[25]}{\langle 13\rangle\langle 32]}+1
\end{array} ; 1\right] \\
= & \frac{1}{k_{1}^{3}}\left(\frac{s_{34}\langle 13\rangle[32]}{\langle 51\rangle[25]}+\frac{s_{35}}{1+\frac{\langle 51\rangle[25]}{\langle 13\rangle\langle 32]}}\right) \alpha^{\prime}+\mathcal{O}\left(\alpha^{\prime 2}\right),
\end{aligned}
$$

and

$$
\begin{aligned}
\tilde{f}_{2}= & \frac{(-1)^{k_{1}}}{\left(k_{1}-1\right) !} \frac{1}{\alpha^{\prime} s_{23}+k_{1}} \frac{\Gamma\left(\alpha^{\prime} s_{51}+\left(\alpha^{\prime} s_{23}+k_{1}\right) \frac{\langle 51\rangle[25]}{\langle 13\rangle\langle 32]}+1\right) \Gamma\left(\alpha^{\prime} s_{34}+1\right) \Gamma\left(\alpha^{\prime} s_{45}+1\right)}{\Gamma\left(-k_{1}+2+\alpha^{\prime} s_{34}\right) \Gamma\left(\alpha^{\prime} s_{51}+\alpha^{\prime} s_{45}+\left(\alpha^{\prime} s_{23}+k_{1}\right) \frac{\langle 51\rangle[25]}{\langle 13\rangle[32]}+2\right)} \\
& \times{ }_{3} F_{2}\left[\begin{array}{c}
-k_{1}+1, \alpha^{\prime} s_{51}+\left(\alpha^{\prime} s_{23}+k_{1}\right) \frac{\langle 51\rangle[25]}{\langle 13\rangle[32]}+1,-\alpha^{\prime} s_{35}+1 \\
-k_{1}+2+\alpha^{\prime} s_{34}, \alpha^{\prime} s_{51}+\alpha^{\prime} s_{45}+\left(\alpha^{\prime} s_{23}+k_{1}\right) \frac{\langle 51\rangle[25]}{\langle 13\rangle\langle 32]}+2
\end{array} ; 1\right] \\
= & -\frac{1}{k_{1}^{2}} \frac{1}{1+\frac{\langle 51\rangle[25]}{\langle 13\rangle[32]}}+\mathcal{O}\left(\alpha^{\prime}\right)
\end{aligned}
$$

leading to the following $\alpha^{\prime}$ expansion of the residue at $z=\frac{\alpha^{\prime} s_{23}+k_{1}}{\alpha^{\prime}\langle 13\rangle[32]}$

$$
\begin{aligned}
& \operatorname{Res}\left(\frac{A_{5}(z)}{z}, z=\frac{\alpha^{\prime} s_{23}+k_{1}}{\alpha^{\prime}\langle 13\rangle\langle 32]}\right)=\frac{\alpha^{\prime 2}}{k_{1}^{2}} \frac{\langle 12\rangle^{4}}{\langle 12\rangle\langle 23\rangle\langle 34\rangle\langle 45\rangle\langle 51\rangle}\left[s_{23} \frac{\langle 51\rangle[25]}{\langle 13\rangle[32]}\left(\frac{s_{34}\langle 13\rangle[32]}{\langle 51\rangle[25]}+\frac{s_{35}}{1+\frac{\langle 51\rangle \mid 25]}{\langle 13\rangle\langle 32]}}\right)\right. \\
& \left.-[12]\langle 23\rangle[35]\langle 51\rangle \frac{1}{1+\frac{\langle 51\rangle[25]}{\langle 13\rangle(32]}}\right]+\mathcal{O}\left(\alpha^{\prime 3}\right)
\end{aligned}
$$

- at $z=\frac{\alpha^{\prime} s_{51}+k_{2}}{\alpha^{\prime}\langle 15\rangle[25]}, k_{2} \in \mathbb{N}$, with residue

$$
\operatorname{Res}\left(\frac{A_{5}(z)}{z}, z=\frac{\alpha^{\prime} s_{51}+k_{2}}{\alpha^{\prime}\langle 15\rangle\langle 25]}\right)=\frac{\langle 12\rangle^{4}}{\langle 12\rangle\langle 23\rangle\langle 34\rangle\langle 45\rangle\langle 51\rangle}\left[-\alpha^{\prime} k_{2} s_{23} \widetilde{f}_{1}+\alpha^{\prime 2}[12]\langle 23\rangle[35]\langle 51\rangle \widetilde{f}_{2}\right],
$$

where

$$
\begin{aligned}
& \tilde{f}_{1}=\frac{(-1)^{k_{2}+1}}{k_{2} !} \frac{1}{\alpha^{\prime} s_{51}+k_{2}} \frac{\Gamma\left(\alpha^{\prime} s_{23}-\left(\alpha^{\prime} s_{51}+k_{2}\right) \frac{\langle 13\rangle[32]}{\langle 15\rangle[25]}\right) \Gamma\left(\alpha^{\prime} s_{34}+1\right) \Gamma\left(\alpha^{\prime} s_{45}+1\right)}{\Gamma\left(-k_{2}+1+\alpha^{\prime} s_{45}\right) \Gamma\left(\alpha^{\prime} s_{23}+\alpha^{\prime} s_{34}-\left(\alpha^{\prime} s_{51}+k_{2}\right) \frac{\langle 13\rangle[32]}{\langle 15\rangle[25]}+1\right)} \\
& \times{ }_{3} F_{2}\left[\begin{array}{c}
\alpha^{\prime} s_{23}-\left(\alpha^{\prime} s_{51}+k_{2}\right) \frac{\langle 13\rangle[32]}{\langle 15\rangle \mid[25]},-k_{2},-\alpha^{\prime} s_{35} \\
\alpha^{\prime} s_{23}+\alpha^{\prime} s_{34}-\left(\alpha^{\prime} s_{51}+k_{2}\right) \frac{\langle 3\rangle \mid[32]}{\langle 15\rangle(25]}+1,-k_{2}+1+\alpha^{\prime} s_{45}
\end{array} ; 1\right] \\
& =\frac{1}{k_{2}^{3}}\left(-\frac{s_{45}\langle 15\rangle[25]}{\langle 13\rangle[32]}+\frac{s_{35}}{1-\frac{\langle 13\rangle[32]}{\langle 15\rangle(25]}}\right) \alpha^{\prime}+\mathcal{O}\left(\alpha^{\prime 2}\right),
\end{aligned}
$$


and

$$
\begin{aligned}
\widetilde{f}_{2}= & \frac{(-1)^{k_{2}}}{\left(k_{2}-1\right) !} \frac{1}{\alpha^{\prime} s_{51}+k_{2}} \frac{\Gamma\left(\alpha^{\prime} s_{23}-\left(\alpha^{\prime} s_{51}+k_{2}\right) \frac{\langle 13\rangle[32]}{\langle 15\rangle[25]}+1\right) \Gamma\left(\alpha^{\prime} s_{34}+1\right) \Gamma\left(\alpha^{\prime} s_{45}+1\right)}{\Gamma\left(-k_{2}+2+\alpha^{\prime} s_{45}\right) \Gamma\left(\alpha^{\prime} s_{23}+\alpha^{\prime} s_{34}-\left(\alpha^{\prime} s_{51}+k_{2}\right) \frac{\langle 13\rangle[32]}{\langle 15\rangle[25]}+2\right)} \\
& \times{ }_{3} F_{2}\left[\begin{array}{c}
\alpha^{\prime} s_{23}-\left(\alpha^{\prime} s_{51}+k_{2}\right) \frac{\langle 13\rangle[32]}{\langle 15\rangle[25]}+1,-k_{2}+1,-\alpha^{\prime} s_{35}+1 \\
\alpha^{\prime} s_{23}+\alpha^{\prime} s_{34}-\left(\alpha^{\prime} s_{51}+k_{2}\right) \frac{\langle 13\rangle[32]}{\langle 15\rangle[25]}+2,-k_{2}+2+\alpha^{\prime} s_{45}
\end{array} ; 1\right] \\
= & -\frac{1}{k_{2}^{2}} \frac{1}{1-\frac{\langle 13\rangle[32]}{\langle 15\rangle\langle 25]}}+\mathcal{O}\left(\alpha^{\prime}\right),
\end{aligned}
$$

leading to the following $\alpha^{\prime}$ expansion of the residue at $z=\frac{\alpha^{\prime} s_{51}+k_{2}}{\alpha^{\prime}\langle 15\rangle[25]}$

$$
\begin{aligned}
& \operatorname{Res}\left(\frac{A_{5}(z)}{z}, z=\frac{\alpha^{\prime} s_{51}+k_{2}}{\left.\alpha^{\prime}\langle 15\rangle \mid 25\right]}\right)=\frac{\alpha^{\prime 2}}{k_{2}^{2}} \frac{\langle 12\rangle^{4}}{\langle 12\rangle\langle 23\rangle\langle 34\rangle\langle 45\rangle\langle 51\rangle}\left[s_{23}\left(\frac{s_{45}\langle 15\rangle[25]}{\langle 13\rangle[32]}-\frac{s_{35}}{1-\frac{\langle 13\rangle[32]}{\langle 15\rangle \mid 25]}}\right)\right. \\
& \left.-[12]\langle 23\rangle[35]\langle 51\rangle \frac{1}{1-\frac{\langle 13\rangle[32]}{\langle 15\rangle(25]}}\right]+\mathcal{O}\left(\alpha^{\prime 3}\right)
\end{aligned}
$$

Adding the residues (C.5), (C.11), (C.17) one obtains ${ }^{18}$

$$
\begin{aligned}
& \operatorname{Res}\left(\frac{A_{5}(z)}{z}, z=\frac{\langle 23\rangle}{\langle 13\rangle}\right)+\sum_{k_{1}=1}^{\infty} \operatorname{Res}\left(\frac{A_{5}(z)}{z}, z=\frac{\alpha^{\prime} s_{23}+k_{1}}{\left.\alpha^{\prime} \backslash 13\right\rangle\langle 32]}\right)+\sum_{k_{2}=1}^{\infty} \operatorname{Res}\left(\frac{A_{5}(z)}{z}, z=\frac{\alpha^{\prime} s_{51}+k_{2}}{\left.\alpha^{\prime} \backslash 15\right\rangle\langle 25]}\right) \\
& =-\frac{\langle 12\rangle^{4}}{\langle 12\rangle\langle 23\rangle\langle 34\rangle\langle 45\rangle\langle 51\rangle}\left(1-\frac{\pi^{2} \alpha^{\prime 2}}{12}\left(s_{12} s_{23}+s_{23} s_{34}+s_{34} s_{45}+s_{45} s_{51}+s_{51} s_{12}+4 i \epsilon(1,2,3,4)\right)+\mathcal{O}\left(\alpha^{\prime 3}\right)\right)
\end{aligned}
$$

which is consistent with [7], eqs. (37) and (39). In particular this implies that we have explicitly shown that up to order $\mathcal{O}\left(\alpha^{\prime 3}\right)$ the residue at infinity is absent,

$$
\oint_{\infty} \frac{A_{5}(z)}{z} d z=\mathcal{O}\left(\alpha^{\prime 3}\right)
$$

The argument in the text proves this to all orders.

\section{References}

[1] E. Witten, Perturbative gauge theory as a string theory in twistor space, Commun. Math. Phys. 252 (2004) 189-258, hep-th/0312171.

[2] F. Cachazo, P. Svrček, and E. Witten, MHV vertices and tree amplitudes in gauge theory, JHEP 09 (2004) 006, hep-th/0403047.

[3] R. Britto, F. Cachazo, and B. Feng, New recursion relations for tree amplitudes of gluons, Nucl. Phys. B715 (2005) 499-522, hep-th/0412308.

[4] R. Britto, F. Cachazo, B. Feng, and E. Witten, Direct proof of tree-level recursion relation in yang-mills theory, Phys. Rev. Lett. 94 (2005) 181602, hep-th/0501052.

[5] S. J. Parke and T. R. Taylor, An amplitude for n gluon scattering, Phys. Rev. Lett. 56 (1986) 2459 .

[6] S. Stieberger and T. R. Taylor, Amplitude for n-gluon superstring scattering, Phys. Rev. Lett. 97 (2006) 211601, hep-th/0607184.

\footnotetext{
${ }^{18}$ Using the identity $[12]\langle 23\rangle[35]\langle 51\rangle=\frac{1}{2}\left(s_{23} s_{34}+s_{45} s_{51}-s_{12} s_{23}-s_{34} s_{45}-s_{51} s_{12}\right)-2 i \epsilon(1,2,3,4)$.
} 
[7] S. Stieberger and T. R. Taylor, Multi-gluon scattering in open superstring theory, Phys. Rev. D74 (2006) 126007, hep-th/0609175.

[8] S. Stieberger and T. R. Taylor, Supersymmetry relations and MHV amplitudes in superstring theory, arXiv:0708.0574 [hep-th].

[9] N. Berkovits and J. Maldacena, Fermionic T-Duality, Dual Superconformal Symmetry, and the Amplitude/Wilson Loop Connection, 0807.3196.

[10] Z. Bern, J. J. M. Carrasco, H. Johansson, and D. A. Kosower, Maximally supersymmetric planar Yang-Mills amplitudes at five loops, Phys. Rev. D76 (2007) 125020, 0705.1864].

[11] L. F. Alday and J. M. Maldacena, Gluon scattering amplitudes at strong coupling, JHEP 06 (2007) 064, 0705.0303.

[12] L. J. Dixon, Calculating scattering amplitudes efficiently, hep-ph/9601359.

[13] M. B. Green, J. H. Schwarz, and E. Witten, Superstring Theory. Vol. 1: Introduction. Cambridge, Uk: Univ. Pr. (1987) 469 p. (Cambridge Monographs On Mathematical Physics).

[14] G. Veneziano, Construction of a crossing-symmetric, Regge behaved amplitude for linearly rising trajectories, Nuovo. Cim. A57 (1968) 190-197.

[15] R. Medina, F. T. Brandt, and F. R. Machado, The open superstring 5-point amplitude revisited, JHEP 07 (2002) 071, hep-th/0208121.

[16] D. Oprisa and S. Stieberger, Six gluon open superstring disk amplitude, multiple hypergeometric series and Euler-Zagier sums, hep-th/0509042.

[17] A. A. Tseytlin, Born-Infeld action, supersymmetry and string theory, hep-th/9908105.

[18] P. Koerber and A. Sevrin, The non-abelian Born-Infeld action through order $\alpha^{\prime 3}$, JHEP 10 (2001) 003, hep-th/0108169.

[19] M. L. Mangano and S. J. Parke, Multiparton amplitudes in gauge theories, Phys. Rept. 200 (1991) 301-367, hep-th/0509223.

[20] M. T. Grisaru and H. N. Pendleton, Some properties of scattering amplitudes in supersymmetric Theories, Nucl. Phys. B124 (1977) 81.

[21] F. Cachazo and P. Svrček, Lectures on twistor strings and perturbative Yang-Mills theory, PoS RTN2005 (2005) 004, hep-th/0504194.

[22] V. P. Nair, A current algebra for some gauge theory amplitudes, Phys. Lett. B214 (1988) 215.

[23] R. Boels, L. Mason, and D. Skinner, Supersymmetric gauge theories in twistor space, JHEP 02 (2007) 014, hep-th/0604040].

[24] R. Boels and C. Schwinn, Deriving CSW rules for massive scalar legs and pure Yang-Mills loops, JHEP 07 (2008) 007, 0805.1197.

[25] N. J. A. Sloane, "The on-line encyclopedia of integer sequences." published electronically at www.research.att.com/ njas/sequences/, 2007.

[26] M. de Roo and M. G. C. Eenink, The effective action for the 4-point functions in abelian open superstring theory, JHEP 08 (2003) 036, hep-th/0307211].

[27] N. Wyllard, Derivative corrections to D-brane actions with constant background fields, Nucl. Phys. B598 (2001) 247-275, hep-th/0008125. 
[28] A. A. Rosly and K. G. Selivanov, Helicity conservation in Born-Infeld theory, hep-th/0204229.

[29] M. B. Green and M. Gutperle, Effects of D-instantons, Nucl. Phys. B498 (1997) 195-227, hep-th/9701093.

[30] G. Chalmers and W. Siegel, The self-dual sector of QCD amplitudes, Phys. Rev. D54 (1996) 7628-7633, hep-th/9606061.

[31] L. J. Mason and D. Skinner, An ambitwistor Yang-Mills Lagrangian, Phys. Lett. B636 (2006) 60-67, hep-th/0510262.

[32] M. Rocek and A. A. Tseytlin, Partial breaking of global D $=4$ supersymmetry, constrained superfields, and 3-brane actions, Phys. Rev. D59 (1999) 106001, hep-th/9811232.

[33] F. Wilczek, Decays of heavy vector mesons into Higgs particles, Phys. Rev. Lett. 39 (1977) 1304.

[34] Z. Bern, L. J. Dixon, and D. A. Kosower, On-shell recurrence relations for one-loop QCD amplitudes, Phys. Rev. D71 (2005) 105013, hep-th/0501240.

[35] N. Arkani-Hamed and J. Kaplan, On Tree Amplitudes in Gauge Theory and Gravity, JHEP 04 (2008) 076, 0801.2385.

[36] P. Di Vecchia, The birth of string theory, Lect. Notes Phys. 737 (2008) 59-118, 0704.0101.

[37] A. Erdelyi, Higher Transcendental Functions Volume 1. 1953. the Bateman Manuscript project.

[38] P. Benincasa and F. Cachazo, Consistency Conditions on the S-Matrix of Massless Particles, 0705.4305 .

[39] F. Liu, Massive amplitudes of the open superstring, Phys. Rev. D38 (1988) 1334.

[40] Z. Xiao and C.-J. Zhu, Factorization and unitarity in superstring theory, JHEP 08 (2005) 058, hep-th/0503248.

[41] K. Risager, A direct proof of the CSW rules, JHEP 12 (2005) 003, hep-th/0508206.

[42] H. Elvang, D. Z. Freedman, and M. Kiermaier, Recursion Relations, Generating Functions, and Unitarity Sums in N=4 SYM Theory, 0808.1720.

[43] R. Boels, L. Mason, and D. Skinner, From twistor actions to MHV diagrams, Phys. Lett. B648 (2007) 90-96, hep-th/0702035.

[44] H. Kawai, D. C. Lewellen, and S. H. H. Tye, A relation between tree amplitudes of closed and open strings, Nucl. Phys. B269 (1986) 1.

[45] O. Lechtenfeld and A. D. Popov, Supertwistors and cubic string field theory for open $N=2$ strings, Phys. Lett. B598 (2004) 113-120, hep-th/0406179.

[46] M. Vonk, A mini-course on topological strings, hep-th/0504147.

[47] N. Arkani-Hamed, F. Cachazo, and J. Kaplan, What is the simplest Quantum Field Theory?, 0808.1446 .

[48] D. Binosi and L. Theussl, JaxoDraw: A graphical user interface for drawing Feynman diagrams, Comput. Phys. Commun. 161 (2004) 76-86, hep-ph/0309015. 